University of Redlands

\title{
Geomapping the Pele-Pillan Region of Jupiter's Moon Io Using Photopolarimeter-Radiometer Data
}

A Major Individual Project submitted in partial satisfaction of the requirements

for the degree of Master of Science in Geographic Information Systems

\author{
by \\ Joyce Marie Hogan \\ Fang Ren, Ph.D., Committee Chair \\ Mark Kumler, Ph.D.
}

September 2009 
Geomapping the Pele-Pillan Region of Jupiter's Moon Io Using Photopolarimeter-Radiometer Data

Copyright (C) 2009

by

Joyce Marie Hogan 
The report of Joyce Marie Hogan is approved.
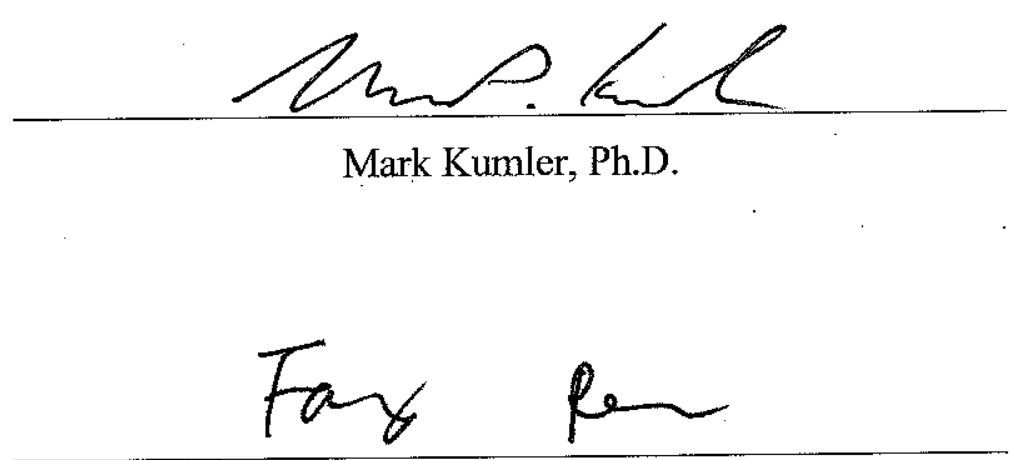

Fang Ren, Ph.D., Committee Chair

September 2009 



\section{Acknowledgements}

I would like to thank James, my mom, and grandpa for their tremendous support.

Special thanks go to Dr. Fang Ren - I feel so lucky to have had her as an advisor. Her guidance, expertise, and kind words helped me in completing this major project. I would also like to thank Dr. Mark Kumler for his interest in my project and his great attention to detail.

Much appreciation goes to Nathan Strout at the Redlands Institute and fellow cohort member, Sean Pack, for their assistance during critical stages of this project. For their time and technical knowledge of VB.NET programming, I cannot thank them enough. Thank you to Dr. Julie Rathbun, who provided me with an "out-of-this-world" project in which to develop my programming skills. Thanks also go to Serene Ong at the Redlands Institute for her GIS knowledge and insight.

I would like to additionally thank Debra Riley, Program Coordinator, for her words of encouragement and for keeping everyone organized. I also want to thank Ruben Ortiz, Brandon Davis, and John Laska for their excellent IT support.

To Cohort 14 - I can't imagine a better group of people to have shared this experience with. The MS GIS faculty and my fellow cohort members have made this a very rewarding, fun, and intense year of learning.

Thank you all!

I dedicate this MIP in loving memory to my dad. 



\author{
Abstract \\ Geomapping the Pele-Pillan Region of Jupiter’s Moon Io \\ Using Photopolarimeter-Radiometer Data \\ by \\ Joyce Marie Hogan
}

The Pele-Pillan region of Jupiter's moon Io is an area of high volcanic activity. The active lava lake Pele and the characteristic fire fountain and turbulent flows of Pillan produce some of the highest thermal outputs for this moon. Planetary scientists do not currently have a means of efficiently processing the surface temperature data captured of this region for incorporation into a geographic information system (GIS). A GIS of the Photopolarimeter-Radiometer (PPR) surface temperature data, that was captured in the late 1990s and the early part of this decade by the Galileo spacecraft, can aid in the visualization and analysis of volcanic activity on Jupiter's moon Io. Current PPR data processing techniques required enhancement and an automated workflow was needed in order to create a complete GIS for Io by the United States Geological Survey deadline of July 2011. This paper focuses on the development of a prototype ArcGIS toolkit for processing PPR data of Io's highly active Pele-Pillan region. The toolkit uses Visual Basic and ArcObjects programming components to: 1) create features in ArcGIS, representing temperature scans of Io's surface, and 2) generate new color-coded, temperature-specific symbology for these features. 



\section{Table of Contents}

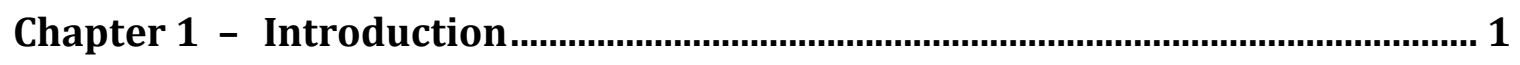

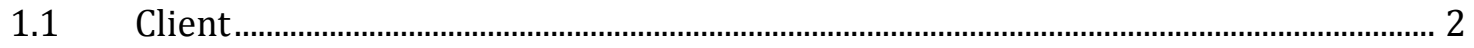

$1.2 \quad$ Problem Statement .......................................................................................................... 2

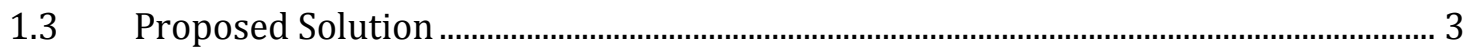

1.3.1 Goals and Objectives ..................................................................................................... 3

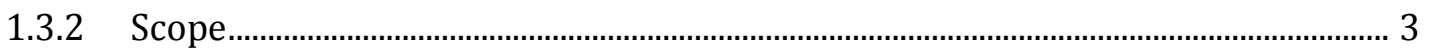

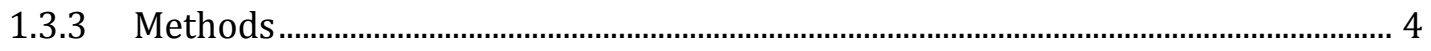

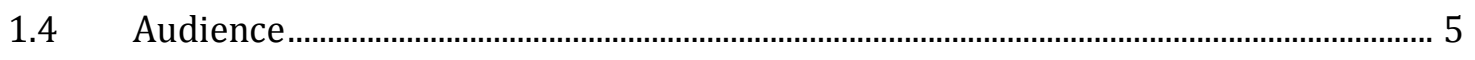

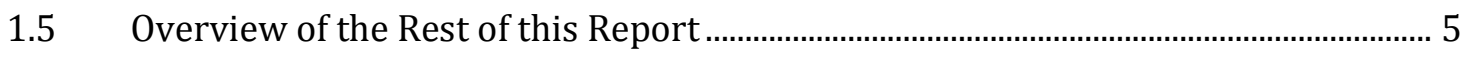

Chapter 2 - Background and Literature Review …............................................... 7

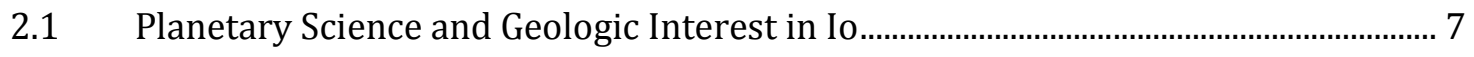

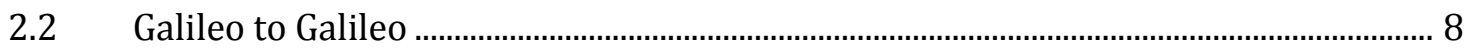

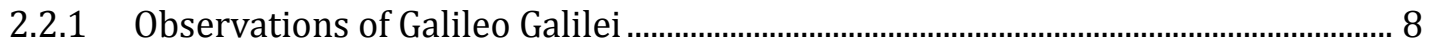

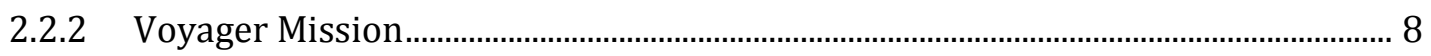

2.2.3 Galileo Mission.................................................................................................................. 9

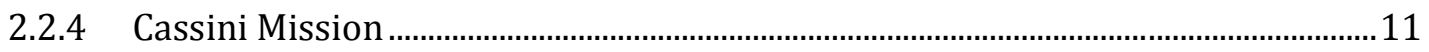

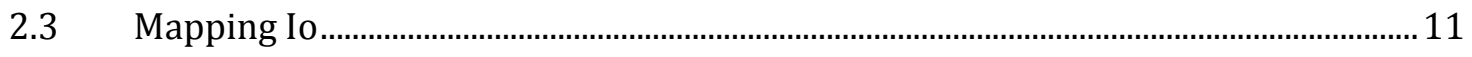

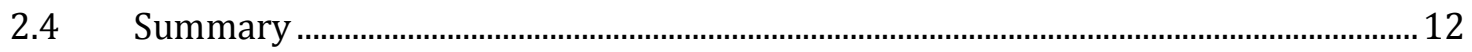

Chapter 3 - Systems Analysis and Design ………...........................................15

3.1 Problem Statement ..............................................................................................

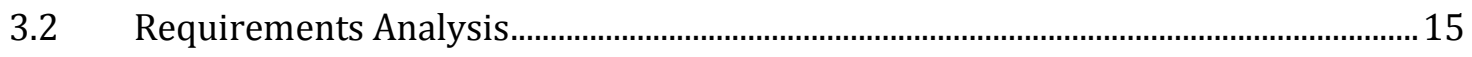

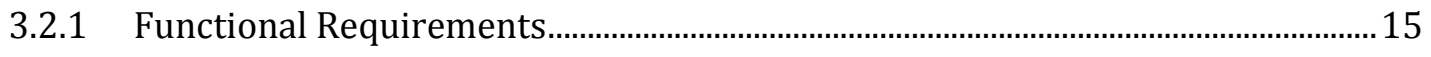

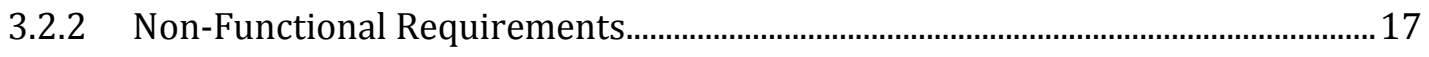

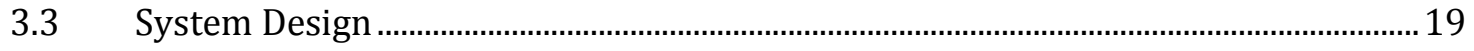

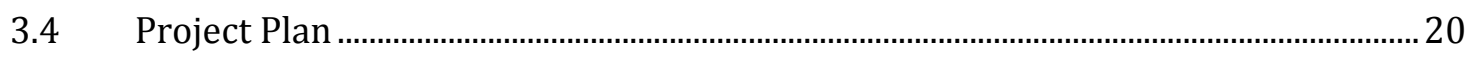

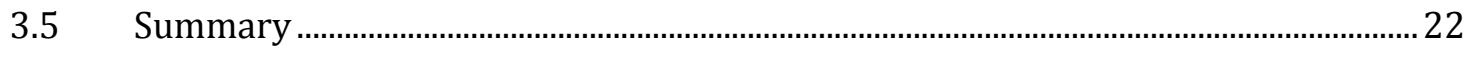

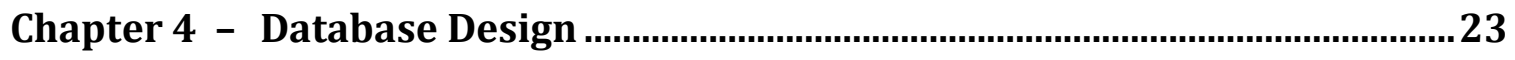

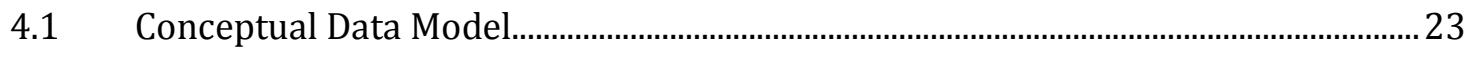

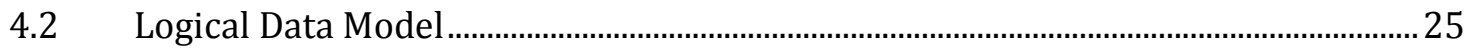

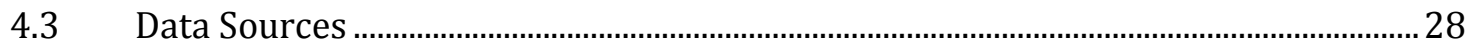

4.4 Data Scrubbing and Loading ......................................................................................

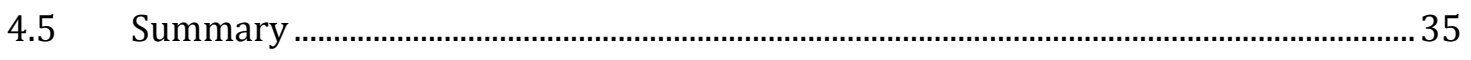

Chapter 5 - Implementation .................................................................................37

5.1 Transformation of Coordinate Points into IFOV Ellipses............................................37

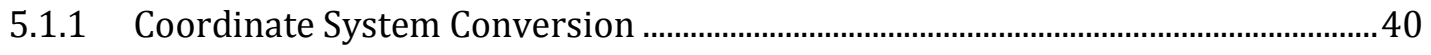

5.1.2 Photopolarimeter-Radiometer Instantaneous Field of View ...................................4 40

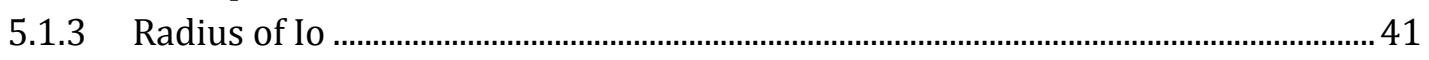




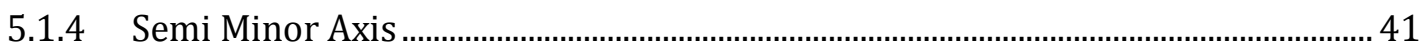

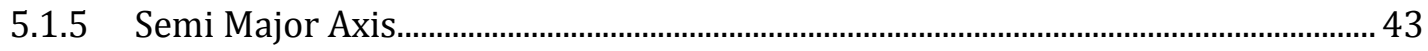

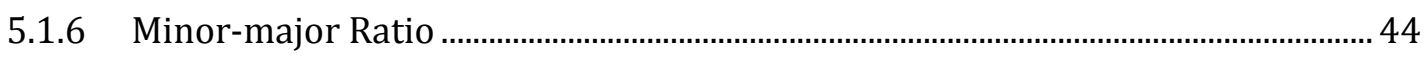

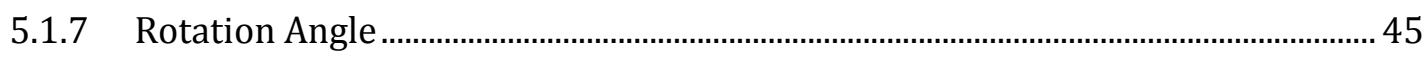

5.1.8 Summary of IFOV Ellipse Parameters........................................................................ 45

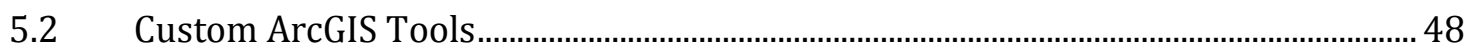

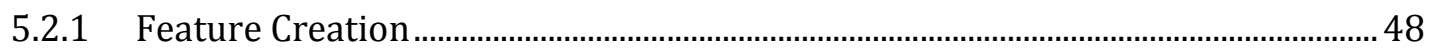

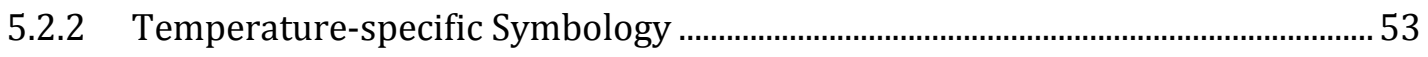

5.2.3 Custom Install Program .................................................................................................. 57

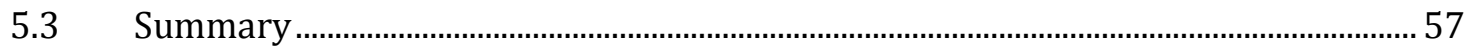

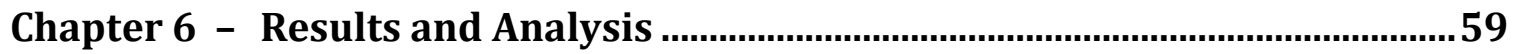

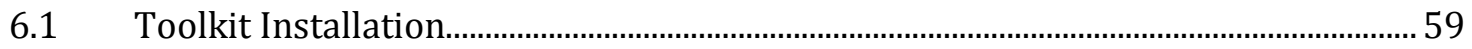

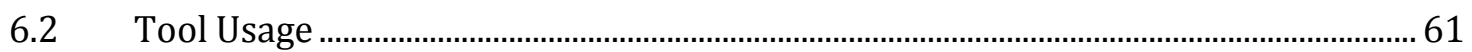

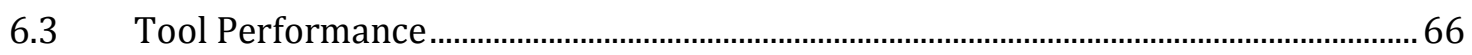

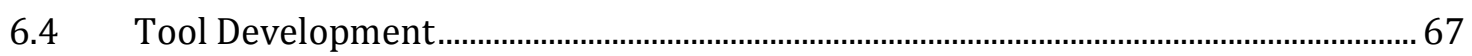

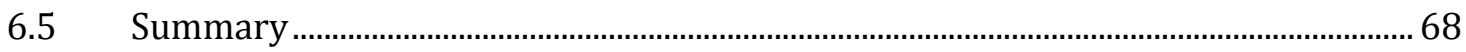

Chapter 7 - Conclusions and Future Work ............................................................69

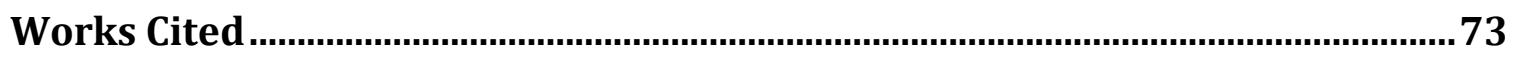

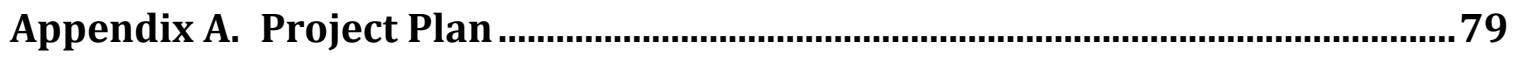

Appendix B. Visual Basic Code for Io PPR Data Processing Toolkit ....................81

Appendix C. "Create a command by inheriting from BaseCommand" Documentation from the ESRI Resource Center .......................... 111

Appendix D. “How to create a custom install program” Documentation from the ESRI Resource Center .................................................. 129 


\section{Table of Figures}

Figure 2-1: The first two volcanic eruption plumes observed on Io..................... 9

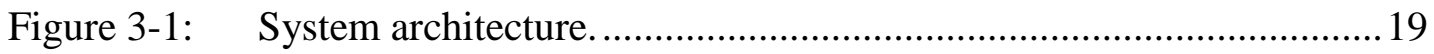

Figure 4-1: $\quad$ The four principal UML shapes used in information system

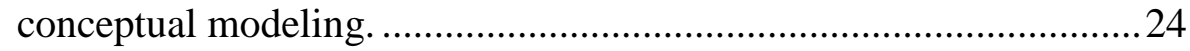

Figure 4-2: $\quad$ UML relationship diagram for PPR data......................................25

Figure 4-3: $\quad$ Logical model for PPR file geodatabase.......................................27

Figure 4-4: Geometry file download process. ..............................................29

Figure 4-5: Subset of Io’s surface feature annotation...................................... 32

Figure 4-6: Initial plotting of data points in ArcGIS Io Geographic Coordinate

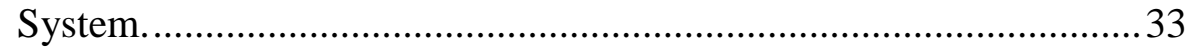

Figure 4-7: $\quad$ Plotting of data points, with adjusted longitude values, in ArcGIS Io Geographic Coordinate System............................................... 34

Figure 4-8: $\quad$ PPR data points plotted on surface of Io, using an azimuthal equidistant projection............................................................... 34

Figure 5-1: $\quad$ Field values in PPR geometry files used to calculate ellipse

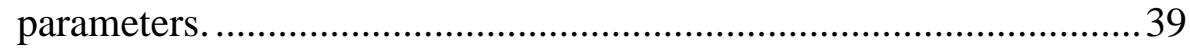

Figure 5-2: $\quad$ Instantaneous field of view of spacecraft instrument. ..................... 41

Figure 5-3: Minor axis of IFOV ellipse. The semi minor axis is half this length................................................................................ 42

Figure 5-4: Tangent of a right triangle........................................................ 42

Figure 5-5: $\quad$ Major axis of IFOV ellipse. The semi major axis is half this length.

Figure 5-6: $\quad$ Minor-major ratio of IFOV ellipse. ............................................. 44

Figure 5-7: $\quad$ Rotation angle of IFOV ellipse................................................. 45

Figure 5-8: $\quad$ Create Features dialog box....................................................... 49

Figure 5-9: Average temperature values in PPR raw data records (.tab files) associated with IFOV ellipses.....................................................50 
Figure 5-10: Buffer.bmp tool button image. ................................................... 52

Figure 5-11: Set Temperature Symbology dialog box..................................... 54

Figure 5-12: $\quad$ Temperature color ramp with associated RGB values. ..................... 55

Figure 5-13: New temperature-specific symbology of a selected layer................ 56

Figure 5-14: Altered Buffer.bmp tool button image. ........................................ 57

Figure 6-1: Dialog box screenshot showing how to add the Io PPR Data

Processing toolbar to ArcMap....................................................... 60

Figure 6-2: Dialog box screenshot showing how to add the Create Features and Set Temperature Symbology tools to an ArcMap toolbar................. 60

Figure 6-3: Dialog box screenshot showing how to add the Create Features tool to an ArcCatalog toolbar. ........................................................... 61

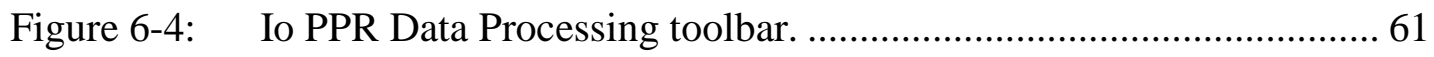

Figure 6-5: Possible types of error messages from the Create Features tool. ...... 62

Figure 6-6: $\quad$ Ellipse and center point features in ArcMap................................. 63

Figure 6-7: $\quad$ New temperature-specific symbology in ArcMap. ........................... 65 


\section{List of Tables}

Table 1. Functional requirements for the Io PPR Data Processing toolkit. ................ 16

Table 2. Non-functional requirements for the Io PPR Data Processing toolkit.......... 17

Table 3. Latitude and longitude parameters for Io Geographic Coordinate Systems

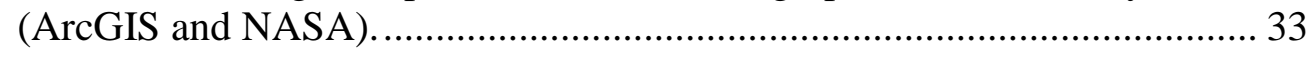

Table 4. Parameters necessary to create an ellipse. ............................................... 38

Table 5. Data, calculations, and factors used to compute ellipse parameters............ 47 



\section{List of Acronyms and Definitions}

$\begin{array}{ll}\text { FOV } & \text { Field of View } \\ \text { GIS } & \text { Geographic Information System } \\ \text { IDL } & \text { Interactive Display Language } \\ \text { IFOV } & \text { Instantaneous Field of View } \\ \text { JPL } & \text { Jet Propulsion Lab } \\ \text { K } & \text { Kelvin } \\ \text { KM } & \text { Kilometer } \\ \text { MIP } & \text { Major Individual Project } \\ \text { MXD } & \text { ArcMap Document } \\ \text { NASA } & \text { National Aeronautics and Space Administration } \\ \text { NIMS } & \text { Near-Infrared Mapping Spectrometer } \\ \text { PDS } & \text { Planetary Data System } \\ \text { PPR } & \text { Photopolarimeter-Radiometer } \\ \text { RDR } & \text { Raw Data Record } \\ \text { RGB } & \text { Red-Green-Blue } \\ \text { USI } & \text { Unified Modeling Language } \\ \text { USG } & \\ \text { Sold State Imager }\end{array}$





\section{Chapter 1 - Introduction}

Remotely-sensed planetary surface data captured by spacecraft has been used in the planetary sciences to enhance understanding of the planetary bodies. The American Society for Photogrammetry and Remote Sensing defines remote sensing and photogrammetry as "the art, science, and technology of obtaining reliable information about physical objects and the environment, through the process of recording, measuring and interpreting imagery and digital representations of energy patterns derived from noncontact sensor systems” (Colwell, 1997). Through advances in remote sensing technology, large volumes of digital data can be obtained in a fairly easy fashion, while the challenge remains of how to efficiently process the data for visual analysis purposes.

Remotely-sensed planetary surface temperature data that have already been processed and published typically appear as vector or raster graphics, color-coded by temperature range. When mapped onto a planet's geographic and/or projected coordinate systems, along with surface imagery, the temperature graphics provide scientists with a geospatial visualization of the thermal emissions occurring on a planet. Planetary scientists are interested in mapping the entire set of Photopolarimeter-Radiometer (PPR) surface temperature data captured by the Galileo spacecraft for Jupiter's moon, Io. The mapped data can aid in determining geographic areas of new and old eruptions on this volcanically active moon.

A Geographic Information System (GIS) is being used to map the Io PPR data, as it has several advantages over traditional planetary mapping techniques. Most importantly, a GIS has the ability to accurately coregister multiple data layers together. This allows all sets of PPR data to be mapped together, along with other available surface temperature data, such as the Galileo spacecraft's Near-Infrared Mapping Spectrometer and Solid State Imager data. Mapping different temperature data layers together helps to create a more comprehensive visualization of Io's surface.

However, manual processing of the complete set of Io PPR data is time-consuming. The United States Geological Survey (USGS) has also imposed a deadline of 2011 for completion of a GIS containing the full set of processed PPR data for Io. To address these issues, this project successfully created an automated PPR data processing toolkit for use with ArcGIS.

This chapter is comprised of five main sections. The client of the project is first introduced, followed by the general problem addressed by this project, and a summary of the proposed solution, with subsections describing the project goals and objectives, scope, and methodology. The primary audience for this project is then addressed; those who would benefit from the new data processing toolkit described in this paper. Finally, 
a brief summary is provided of the topics covered in the remaining chapters of this project report.

\subsection{Client}

The client for this project is Dr. Julie Rathbun, Ph.D., Associate Professor of Physics at the University of Redlands. Since 1999, she has been working closely with the National Aeronautics and Space Administration Jet Propulsion Lab (NASA JPL), USGS, the Southwest Research Institute, and Arizona State University on various research projects involving observations of Io. These projects have ranged from looking at the history of eruptions from Io's powerful volcano, Loki, using groundbased and Galileo spacecraft data, to observing Io's volcanic activity in the region of Tvashtar Catena, a chain of giant calderas, using Galileo and Cassini spacecraft data. Dr. Rathbun is a definitive expert on volcanism on Jupiter's moon Io. To date, she has published twenty-two first-author and five co-authored scientific journal articles and abstracts on the subject.

Dr. Rathbun expressed the need for a GIS that would incorporate all of the PPR data for Io from the Galileo spacecraft mission. Previously, planetary mappers have used Interactive Display Language (IDL) programming code to map and visualize surface temperature information for a few of the scanned areas of Io. IDL programming, however, has some key problems, in that it:

- requires a significant amount of programming just to provide functionality that ArcGIS already has out-of-the-box

- does not have a graphical user interface for user-friendly mapping, since it is just a programming language

- does not have customization options for map output

Conversely, the GIS environment is user-friendly and robust, with many options for customization, which makes it ideal for planetary mapping.

For this project, Dr. Rathbun requested that an automated procedure be created for use with ArcGIS to process the PPR data in a timely manner, focusing on the volcanically active Pele-Pillan region of Io. This automated procedure will be used in the near future to process PPR data for all other regions of Io in order to meet the USGS deadline of 2011 for incorporating all PPR data for Io into a GIS.

\subsection{Problem Statement}

Currently, planetary geologists and associated scientists do not have a means of efficiently processing the PPR surface temperature data captured by the Galileo spacecraft for incorporation into a GIS. A GIS of PPR data would enable accurate visualization and analysis of the volcanic activity on Jupiter's moon Io. The present 
method for processing PPR data is quite lengthy and ineffective for processing a large number of datasets. A solution was needed that could automate the PPR data processing. Improving the current technique further helps in meeting the USGS deadline of July 2011 for completion of a GIS containing the entire set of PPR data for Io.

\subsection{Proposed Solution}

This section discusses the general approach to solving the problem statement described in Section 1.2. It provides details on the goals and objectives of the project. The project scope and methodology are further outlined based on these objectives.

\subsubsection{Goals and Objectives}

The goal of this project was to develop a toolkit to process Io PPR surface temperature data for visualization in a GIS. The USGS deadline of 2011 has further pushed this project into priority status. In keeping with this goal, the main project objective was to create a toolkit for ArcGIS that accurately and efficiently processes PPR data with automated procedures, requiring minimal user input. The user only has to specify the files to process and the location where the newly created geodatabase files are to be saved. The key was not to sacrifice accuracy for automation. The second objective of the project was to create an intuitive user interface for the toolkit, using a simple Windows form design with a minimal number of text boxes and buttons. This helps to ensure ease of use for first time users who are somewhat familiar with the raw PPR data files. The third objective of the project was to be able to share the tool with other users, most likely through the internet. Creating the tool as a portable file type for distribution makes it possible for any potential user to easily download the tool from a future website, and start using the tool immediately on a Windows operating system that has ArcGIS installed. The fourth objective of the project was to process a subset of Io PPR data using the newly developed tool. This processed data will ultimately be included in a GIS that will contain the entire set of Io PPR data.

\subsubsection{Scope}

In order to develop and test the automated processing procedures to incorporate PPR data into ArcGIS, a subregion of Io was chosen. Consequently, the data for this project solely focuses on the Pele-Pillan region of Io, an area of intense volcanic activity.

The final product of the project is a set of custom ArcGIS tools that realize the automated processing procedures. The tools were developed using the .NET framework and written in Visual Basic programming language. ArcObjects programming components were utilized to create tool functionality in the ArcGIS environment. Persons who are involved with the PPR data processing for Io into ArcGIS will be able to install the custom toolkit directly from an executable file. Once the toolbar and tools are 
initialized through the Tools $\rightarrow$ Customize menu in ArcMap and ArcCatalog, a GIS user can utilize the functionality of the custom tools in ArcGIS.

The new ArcGIS toolbar, named Io PPR Data Processing, houses the two custom tools: the Create Features tool and the Set Temperature Symbology tool. The Create Features tool uses spherical geometry calculations to create an instantaneous field of view (IFOV) ellipse and corresponding center point feature for each PPR data record. An IFOV ellipse represents the area scanned on the ground surface of Io at that particular moment in time, based on the angle of view sensed by the Photopolarimeter-Radiometer and the sensor's location. Attribute tables are also created containing each IFOV ellipse's temperature reading. The Set Temperature Symbology tool creates new colorcoded symbology for the ellipses, based on these temperature readings.

Additional user functionality includes map tips and tool tips, which display when the mouse hovers over a tool button, and message boxes that inform the user of any specific errors and of the completion of processing. Spatial averaging of the temperature data for overlapping and proximate ellipses, however, is not included in the functionality of the toolkit, as it was out of the project scope agreed upon with the client.

This project was conceived as a prototype toolkit for processing the entire set of PPR data. As such, it is expected that continued use of the tool by the planetary GIS community will spur revisions to the automated procedures.

\subsubsection{Methods}

The particular methods used to complete this project included conducting a requirements analysis, gathering \& understanding the data, development of a toolkit for processing the PPR data, and delivery of the completed toolkit, geodatabase of processed data, and ArcMap symbology to the client. The requirements analysis involved a client needs assessment and creation of a project plan in order to manage the completion of key project tasks. The next step, gathering of the necessary data, included compiling the PPR data files for the Pele-Pillan region and ancillary reference files, including a background image of Io's surface, a latitude-longitude grid, and surface feature annotation for Io. Understanding the data was instrumental to identifying and addressing data issues. Subsequent development of an ArcGIS toolkit, for processing the raw PPR data files, entailed identifying and understanding programming methods and calculations for creating new ellipses and center point features, attribute tables containing the important temperature data, and new temperature-specific symbology. The custom interfaces for the toolkit were designed to be easy-to-use, with simple layouts and clear instructions, facilitating the efficient programmatic processing of raw PPR data files with just a few button clicks. The completed toolkit was wrapped up as an executable file and provided to the client. For data organization and storage, a geodatabase was also created and 
delivered to the client, containing the processed PPR ellipses and center point feature classes for Io's Pele-Pillan region and the ancillary reference files. Lastly, the client was provided with an ArcMap document (.mxd file) linked to these files, displaying the new temperature-specific symbology of the ellipse features.

\subsection{Audience}

This paper addresses the need of researchers and GIS professionals in the field of planetary science for an efficient method to process text file data for visualization and further analysis. Much of the data that NASA has collected from its spacecraft missions is in text file format and needs to be manipulated specifically for data visualization purposes. Planetary scientists and GIS professionals will be able to improve workflow by incorporating all or selected features of the developed ArcGIS toolkit, described in this document, to save time in the complicated processing of text file data for incorporation into a GIS. The toolkit will be most relevant to those involved with processing PPR data for other regions of Io. GIS professionals and developers, who process PPR data or any text file-formatted data for other planetary bodies, may be interested in select aspects of the tool's automated processes.

\subsection{Overview of the Rest of this Report}

This report is comprised of six additional chapters. Chapter Two provides background for the project and a review of pertinent literature from authoritative sources. Chapter Three outlines the system design considerations for the project. Chapter Four discusses the various data design models that were developed, data download procedures, components of the data, and data preparation. Chapter Five describes the steps taken to complete the project in satisfaction of the stated project goals and objectives. Chapter Six addresses the results of the project implementation. Finally, Chapter Seven presents the general conclusions of the project and suggestions for future work. 



\section{Chapter 2 - Background and Literature Review}

The goal of this project was to improve existing methods for visualizing Io's PPR surface temperature data. A background and literature review was first conducted, from which the solution to the project was proposed and implemented. This chapter introduces the broad field of planetary science and the sub-field of astrogeology as it pertains to Io. In doing so, it attempts to provide background knowledge for the reader. Important observations of Io's volcanism in the Pele-Pillan region are then summarized. Finally, the mapping techniques, used to visualize these observations, are discussed.

\subsection{Planetary Science and Geologic Interest in Io}

Planetary science is the study of planets and planetary systems. Planetary science applies the "theories and methods of traditional disciplines such as astronomy, geology, physics, chemistry, and mathematics to the study of the origin, composition, and distribution of matter in planetary systems, especially the evolution and structure of planets and their natural satellites” (The Columbia Electronic Encyclopedia, 2005). The study of other planets and planetary systems is important for understanding our own planet Earth. A particularly active area of research within the planetary science arena is astrogeology, which includes the study of landforms on celestial bodies, such as boulder-strewn valleys and volcanoes, and the physical processes that create them. This modern science officially came into being in the early 1960s when Eugene Merle Shoemaker, "the father of lunar geology”, coined the term astrogeology (Schaber, 2005). During this period, Shoemaker developed methods for mapping the geology of the moon from telescopic images, and founded the Branch of Astrogeology of the USGS. Studying geological formations and processes on other planetary bodies can provide invaluable clues into the history of geologic processes on early Earth and may offer insight into the timing and intensity of such processes.

Jupiter's moon Io is of much interest to scientists, as it is the most volcanically active body in the solar system (National Aeronautics and Space Administration [NASA], 2008). Furthermore, Io and Earth are the only planetary bodies with active, hightemperature volcanoes. Both Io and Earth have been observed to have magma at temperatures > 1000 kelvin (K) shaping their surfaces (Davies, 2007). Examining Io’s volcanic activity can improve understanding of volcanic processes on Earth. Specifically, study of Io's ultramafic lava (containing less than $45 \%$ silica and a high content of magnesium and iron), can provide insight into the early volcanic activities on Earth during the Precambrian period (Matson, Blaney, Johnson, Veeder, \& Davis, 1998; Williams, et al., 2002). Ultramafic lava is characteristic of very-high temperature eruptions on Io and is akin to the magmas that created komattiites (ultramafic mantlederived volcanic rocks) on Earth, dating back billions of years. 


\subsection{Galileo to Galileo}

\subsubsection{Observations of Galileo Galilei}

Io is one of the four largest moons of Jupiter, discovered by Galileo Galilei; later to be known as one of the Galilean moons. Besides Io, these moons consisted of Europa, Ganymede, and Callisto. Galileo's observations were published in Sidereus Nuncius in March 1610 (Baalke, n.d.):

"I should disclose and publish to the world the occasion of discovering and observing four Planets, never seen from the beginning of the world up to our own times, their positions, and the observations made during the last two months about their movements and their changes of magnitude; and I summon all astronomers to apply themselves to examine and determine their periodic times, which it has not been permitted me to achieve up to this day ... On the 7th day of January in the present year, 1610, in the first hour of the following night, when I was viewing the constellations of the heavens through a telescope, the planet Jupiter presented itself to my view, and as I had prepared for myself a very excellent instrument, I noticed a circumstance which I had never been able to notice before, namely that three little stars, small but very bright, were near the planet; and although I believed them to belong to a number of the fixed stars, yet they made me somewhat wonder, because they seemed to be arranged exactly in a straight line, parallel to the ecliptic, and to be brighter than the rest of the stars, equal to them in magnitude . . When on January 8th, led by some fatality, I turned again to look at the same part of the heavens, I found a very different state of things, for there were three little stars all west of Jupiter, and nearer together than on the previous night."

"I therefore concluded, and decided unhesitatingly, that there are three stars in the heavens moving about Jupiter, as Venus and Mercury around the Sun; which was at length established as clear as daylight by numerous other subsequent observations. These observations also established that there are not only three, but four, erratic sidereal bodies performing their revolutions around Jupiter."

\subsubsection{Voyager Mission}

However, volcanism on Io wouldn't be discovered until over 350 years later. Just prior to Voyager 1's observations of Io, Peale, Cassen, \& Reynolds (1979) published a paper, postulating "widespread and recurrent surface volcanism” on Io. Nearly one month later, the first actual evidence of Io's active volcanism was discovered by Linda A. Morabito (Morabito, Synnott, Kupferman, \& Collins, 1979) in an image captured by the Voyager 1 spacecraft at 13:28 GMT on March 8, 1979, within a 4.5 million kilometer $(\mathrm{km})$ range of Io (Figure 2-1). The first identified eruption plume was an ash cloud above Io's eastern 
limb (in Figure 2-1, North is in the down direction). A second eruption plume was located catching light from the sun on the terminator between day and night. The first plume was later associated with the volcano Pele.

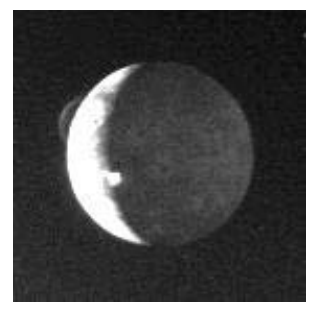

Figure 2-1: The first two volcanic eruption plumes observed on Io.

The Voyager mission consisted of two spacecraft, as described by Stone and Lane (1979a, 1979b). Voyager 1 was launched on September 5, 1977 and made several close fly-bys of Io, with the closest approach at 20,570 kilometers. Voyager 2 (on a slower trajectory) was launched slightly earlier on August 20, 1977, with the closest approach to Io at 1,129,900 kilometers. When compared to images of the Pele plume captured by Voyager 1, the images taken four months later by Voyager 2 revealed that eruptions at Pele had dissipated (Smith, et al., 1979a).

A primary accomplishment of the Voyager mission was the creation of a geologic map of Io from moderate- and high-resolution Voyager 1 images (Masursky, Schaber, Soderblom, \& Strom, 1979; Schaber, 1980, 1982). This map covers approximately a third of Io's surface and includes labels for mountains, plains (inter-vent, layered, and eroded), and vent regions (paterae) (Carr, Masursky, Strom, \& Terrile, 1979; Smith, et al., 1979b; Schaber, 1982).

Another major accomplishment of the Voyager mission was the identification of hot spots on Io, associated with intense volcanism, using Voyager 1's infrared radiometer interferometer and spectrometer (IRIS) data (Smith, et al., 1979b). Thermal spectra of Io, collected by IRIS at wavelengths $4 \mu \mathrm{m}$ to $55 \mu \mathrm{m}$ and covering a third of Io's surface (Hanel, et al., 1979), were coregistered with imagery from Voyager 1 to reveal Io's hot spots. The highest temperature of $654 \mathrm{~K}$ was located at the Pele plume; no other location detected a temperature reading $>600 \mathrm{~K}$.

\subsubsection{Galileo Mission}

The Galileo spacecraft was launched onboard the Space Shuttle Atlantis on October 18, 1989 (NASA, 2001). The spacecraft captured imagery and temperature data for Io from 1996 to 2002 using three sets of instruments: the Solid State Imager (SSI), the Near Infrared Mapping Spectrometer (NIMS), and the Photopolarimeter-Radiometer (PPR) (Davies, 2007). Several close fly-bys of Io were made in October 1999 (orbit I24, closest 
approach $611 \mathrm{~km}$ ), November 1999 (orbit I25, closest approach $301 \mathrm{~km}$ ), February 2000 (orbit I27, closest approach $2013 \mathrm{~km}$ ), and October 2001 (orbit I32, closest approach 182 $\mathrm{km})$.

The Solid State Imager can detect thermal emission from high temperature (> $1000 \mathrm{~K}$ ) sources and produced high spatial resolution images of these hot spots (Rathbun \& Barrett, 2007). These images have resolutions ranging from $64 \mathrm{~km}$ per pixel to $5.5 \mathrm{~m}$ per pixel (typically 10 - $20 \mathrm{~km}$ per pixel), and were obtained using a clear, broad wavelength filter with an effective wavelength of $0.652 \mu \mathrm{m}$ (Davies, 2007).

Additionally, a new geologic map of Io was created from a mosaic of the best SSI and Voyager images, covering the extent of Io (Becker \& Geissler, 2005). Pele, like most of Io's surface features, appeared to have not substantially changed since being imaged by Voyager 2.

However, one of the most noteworthy events was captured through a time-sequence of SSI images: the June 1997 eruption of Pillan (Keszthelyi, et al., 2001). A black area approximately $400 \mathrm{~km}$ in diameter had appeared and images of subsequent orbits revealed that the dark Pillan deposits were being buried by Pele's red plume deposits.

The Near-Infrared Mapping Spectrometer detects thermal emission from active or recently active volcanoes, covering temperatures approximately $180 \mathrm{~K}$ to $>1200 \mathrm{~K}$ (Smythe, et al., 1995; Davies, et al., 2001). These hyperspectral images, captured in absorption bands from $0.7 \mu \mathrm{m}$ to $5.2 \mu \mathrm{m}$, range from low to high spatial resolution for Io (> $200 \mathrm{~km}$ per pixel to $0.5 \mathrm{~km}$ per pixel). NIMS confirmed hot spots that were detected by SSI, as well as new lower-temperature hot spots.

The Photopolarimeter-Radiometer is capable of detecting thermal emission from low-temperature hot spots (i.e. cooling lava flows) at temperatures from about as low as approximately 60 to as high as $700 \mathrm{~K}$ (Rathbun, et al., 2004). The PPR instrument captured thermal emission data in conjunction with much of the SSI and NIMS imagery, mostly in radiometry mode, using a " $17-\mu$ m" filter which effectively covered the range of $14.7 \mu \mathrm{m}$ to $18.9 \mu \mathrm{m}$. These data are of low spatial resolution due to the large instantaneous field of view (2.5 milliradians) of the instrument. One of the main advantages of the PPR data was that it detected additional low temperature thermal sources that were not detectable by SSI or NIMS. Rathbun, Block, and Spencer (2005) suggested that the only way to properly interpret the PPR data is to register the data to the higher resolution SSI and NIMS imagery.

Thus far, comparing thermal activity captured by SSI, NIMS, and PPR has resulted in hypotheses of the types of volcanism present at Pele and Pillan. Pele is suspected to be an active overturning lava lake (Spencer, et al., 2000; Davies, et al., 2001). Hightemperatures have consistently been found at Pele in all orbits, but with no immediate 
cooling lava flows. Pillan, on the other hand, exhibited the characteristics of a lava fountain with associated lava flows (Davies, 2007). The temperatures were observed to increase to peak thermal emission at Pillan, coinciding with the 1997 eruption shown in SSI images. Over the next four years, NIMS and PPR data confirmed the trend of cooling emplaced lava from Pillan.

The Galileo spacecraft mission ended on September 21, 2003 when it disintegrated in Jupiter's atmosphere. The spacecraft was deliberately steered into Jupiter's atmosphere and destroyed, in order to protect a possible ocean beneath the icy crust of the moon Europa (NASA, 2007).

\subsubsection{Cassini Mission}

From the end of 2000 to late 2001, Io was observed by both the Galileo and Cassini spacecrafts. Images of Pele were captured by the Cassini Imaging Sub-System (ISS) on the timescale of several minutes over two hour periods, every 40 hours. The images in the visible wavelength range showed much variation in Pele's activity in the course of a few minutes, which is typical of an active lava lake (Radebaugh, et al., 2004). Images taken through different filters recorded a high temperature reading for Pele of $1500 \mathrm{~K} \pm$ $80 \mathrm{~K}$, suggesting basaltic or ultramafic eruption activity (Radebaugh, et al., 2004).

\subsection{Mapping Io}

Mapping geologic information has been instrumental in understanding the evolution of planetary bodies (Carr, Wilhelms, Greeley, \& Guest, 1976; Carr, Saunders, Strom, \& Wilhelms, 1984; Hansen, 2000; Tanaka, 1994; Wilhelms, 1990). In order to identify the role of impact craters and volcanic, tectonic, and gradational processes in shaping a planet's surface, scientists categorize planetary surface features by specific physical attributes and map the distributions of these features with visible structural features. Due to the availability of large volumes of digital data and improvements in computer graphics hardware and software, mapping techniques have evolved into a new paradigm of geovisualization from traditional map-making. Particularly, geographic information systems (GIS) play an important role in this new paradigm of cartographic visualization, because they are not only an effective application for producing maps, but they are also useful for the storage, creation, manipulation, multi-layered analysis, and visualization of any geographically referenced data (Hare, Tanaka, \& Skinner, 2003).

However, the planetary science community has been slow to incorporate the widespread use of GIS for planetary mapping for several reasons. Planetary mappers are accustomed to creating their own software applications for mapping, as commercial mapping software has historically been geared towards terrestrial mapping (Hare, Tanaka, \& Skinner, 2003). And only recently have planetary researchers started to 
conduct complex spatial analyses by overlaying multiple datasets, a major functionality that the GIS provides (Hare, Tanaka, \& Skinner, 2003). Nearly all maps of Io have been created using Interactive Display Language (IDL) programming code, a staple of planetary scientists (J. Rathbun, personal communication, November 17, 2008). In spite of the long historical use of IDL programming by planetary scientists, there are several disadvantages to creating maps with IDL compared to using ArcGIS software (as mentioned in Chapter 1). The use of IDL requires a significant amount of programming just to provide functionality that ArcGIS already has out-of-the-box. In addition, there is no graphical user interface for user-friendly mapping, since it is just a programming language. IDL also does not provide customization options for map output.

Given the limitations of IDL-based solutions, more advanced technologies have been explored to improve planetary data mapping. For example, Barrett (2006a) produced the first prototype GIS for Io’s Amirani region, incorporating PPR data and accounting for the elliptic instantaneous field of view of the PPR instrument. Although this proof-ofconcept is an excellent starting point for creating a planetary GIS, its usefulness to planetary scientists is limited. The major reason is that this prototype application involved very laborious and time-consuming manual processing of the data.

In order to create a more efficient GIS application for the entire set of PPR data for Io by 2011, an automated process was needed. To fill this gap, this project focused on creating an ArcGIS tool for the automated processing of Io PPR data for incorporation into a GIS. The data for the Pele-Pillan region of Io was selected for use in development of the ArcGIS tool and prototype GIS for Io because it is a hot spot of volcanic activity.

\subsection{Summary}

Io is of great interest to scientists as it is the most volcanically active body in the solar system. However, the traditional methods of displaying the data for Io's surface temperature are not effective and therefore, the USGS Astrogeology Team, under the guidance of the Geologic Mapping Subcommittee, has recognized the need for analysis using digital geospatial mapping applications, such as ArcGIS. The team requires that all geologic maps funded by NASA’s Planetary Geology and Geophysics Program, and submitted for 2011 and later, must be in a GIS-compatible format (Hare, et al., 2009). For Jupiter's moon Io, the USGS has already set a deadline of July 2011 for incorporating all Photopolarimeter-Radiometer (PPR) surface temperature data from the Galileo spacecraft mission into a GIS (J. Rathbun, personal communication, October 11, 2008). Incorporating SSI and NIMS data into this GIS is an important secondary priority. The PPR surface temperature data will be useful in helping to determine geospatial patterns of volcanic activity on Io. With future integration of SSI and NIMS data, the GIS could eventually be used to determine geographic areas of new and old eruptions on Io, using a time-series visualization of all data. Such a GIS can further aid in analyzing Io’s 
complex surface composition. Future studies, combining data from the Galileo mission with data from the recent New Horizons flyby and previous Voyager and Cassini missions, could create a more complete timeline of volcanic activity on Io. 



\section{Chapter 3 - Systems Analysis and Design}

Successful GIS planning involves understanding the needs of both the client and the user, as well as considering the hardware, software, and network components that affect the design of the application. These needs and considerations form the building blocks of a conceptual system design - the foundation for building the final software product. A good system design will minimize problems during implementation and operation. This chapter first revisits the general problem that this project seeks to address. The functional and non-functional requirements for the proposed software system are then discussed, from which a conceptual system design and list of major project tasks were developed. Changes to the project task schedule are also discussed.

\subsection{Problem Statement}

Currently, planetary geologists and associated scientists do not have a means of efficiently processing the PPR surface temperature data captured by the Galileo spacecraft for incorporation into a GIS. A GIS of PPR data would enable accurate visualization and analysis of the volcanic activity on Jupiter's moon Io. The present method for processing PPR data is quite lengthy and ineffective for processing a large number of datasets. A solution was needed that could automate the PPR data processing. Improving the current technique further helps in meeting the USGS deadline of July 2011 for completion of a GIS containing the entire set of PPR data for Io.

\subsection{Requirements Analysis}

Since the PPR data processing could not be achieved with out-of-the-box ArcGIS tools, a new custom built ArcGIS PPR data processing toolkit was deemed to be the appropriate software solution. A requirements analysis was first conducted to determine the specifications of the software system necessary for meeting user needs and complying with technology constraints. These specifications include both functional and nonfunctional requirements.

\subsubsection{Functional Requirements}

Functional requirements take into account the user needs from a system perspective. The functional requirements for the Io PPR Data Processing toolkit are listed in Table 1. These requirements address the access method to the software and the functionality of the toolkit. 
Table 1. Functional requirements for the Io PPR Data Processing toolkit.

\begin{tabular}{|ll|}
\hline $\begin{array}{l}\text { Requirement } \\
\text { environment }\end{array}$ & Description \\
\hline $\begin{array}{l}\text { Inputs data from user-selected PPR text } \\
\text { file pairs }\end{array}$ & $\begin{array}{l}\text { Enables user to run the software from a } \\
\text { desktop or laptop computer } \\
\text { to process }\end{array}$ \\
\hline $\begin{array}{l}\text { Generates instantaneous field of view } \\
\text { (IFOV) ellipses as features (feature } \\
\text { classes or shapefiles) }\end{array}$ & $\begin{array}{l}\text { Enables user to create features in } \\
\text { ArcGIS, representing temperature scans } \\
\text { of Io's surface }\end{array}$ \\
\hline $\begin{array}{l}\text { Saves ellipse features in a user-selected } \\
\text { output workspace }\end{array}$ & $\begin{array}{l}\text { Enables user to select a preexisting } \\
\text { geodatabase (file, personal, or SDE } \\
\text { geodatabase) or file folder in which to } \\
\text { save the features }\end{array}$ \\
\hline Selects an ellipse layer for processing & $\begin{array}{l}\text { Enables user to select an ellipse layer to } \\
\text { symbolize }\end{array}$ \\
\hline $\begin{array}{l}\text { Generates symbolized ellipse layers, } \\
\text { color-coded and labeled by temperature } \\
\text { range }\end{array}$ & $\begin{array}{l}\text { Enables user to create ellipse layer } \\
\text { symbology based on the temperature } \\
\text { values associated with the ellipses }\end{array}$ \\
\hline
\end{tabular}

The client and other potential users of the Io PPR Data Processing Toolkit, including planetary scientists and staff, will be processing PPR data and new feature layers on desktop or laptop computers. The toolkit may need to be installed on multiple machines, so that processing can occur on more than one computer at a time. There is no need for an enterprise system, as this project has a limited lifespan for solely processing data and layers for Io. The client may decide to incorporate an enterprise system for distribution and access to the original text file data and processed features/layers; however, this is out of the project scope.

Users need a tool that processes the original PPR text files for visualization within ArcGIS. Specifically, the original text file data are used to create instantaneous field of view (IFOV) ellipse features, which represent temperature scans of Io's surface captured within the field of view of the PPR instrument at a particular moment in time. In conjunction with this tool function, a user must be able to type in a file path or browse to a specific location to: 1) select the initial files for processing and 2) save the newly processed features to a particular geodatabase or file folder. 
Users also need a tool that allows easy visualization in ArcMap of the approximate surface temperature values associated with each of IFOV ellipses. New symbology needs to be created for the ellipse feature layers based on temperature classification. The new symbology should be color-coded to match the spectrum of temperature values associated with the ellipses. The user should also be able to select a layer for symbolizing from a drop-down list. The list of layers would only contain polygon layers with a valid temperature field.

\subsubsection{Non-Functional Requirements}

Non-functional requirements take into account the technology and user-imposed constraints on a system. The non-functional requirements for the Io PPR Data Processing toolkit encompass the technical, operational, and transitional requirements of the software system (Table 2).

Table 2. Non-functional requirements for the Io PPR Data Processing toolkit.

\begin{tabular}{|ll|}
\hline Requirement & Definition \\
\hline $\begin{array}{l}\text { Technical } \\
\text { specifications for ArcInfo } \\
\text { desktop }\end{array}$ & $\begin{array}{l}\text { PC-Intel platform } \\
\text { Windows Vista (Ultimate, Enterprise, Business, } \\
\text { Home Premium), Windows 2000, or Windows XP } \\
\text { (Home Edition and Professional) operating system }\end{array}$ \\
& 1 GB RAM memory \\
& \begin{tabular}{l}
1.6 GHz processor \\
\hline ArcInfo 9.3
\end{tabular} \\
\hline $\begin{array}{l}\text { Toolkit only works with ArcInfo 9.3, as it was } \\
\text { developed in the ArcInfo 9.3 environment } \\
\text { with clear, concise instructions }\end{array}$ & $\begin{array}{l}\text { User interface for ArcMap and ArcCatalog tools, } \\
\text { designed for users with limited GIS experience, who } \\
\text { are generally familiar with the PPR text files to be } \\
\text { processed }\end{array}$ \\
\hline Portable & $\begin{array}{l}\text { Executable install file allows direct installation of } \\
\text { toolkit onto user's desktop }\end{array}$ \\
\hline
\end{tabular}




\section{Requirement Definition}

\section{Operational}

Quick and efficient processing Automated toolkit functions create new features and symbology quickly, project features in Io_2000 geographic coordinate system, clean-up data

Checks for user input errors \& Message boxes notify a user of any entry errors and confirms completion of the completion of processing processing

\section{Transitional}

Client meeting Brief training on how to use the toolkit

Documentation Detailed MIP report

The technical requirements include the minimum software and hardware specifications necessary to run the toolkit, as well as the critical structural components of the toolkit. In order for the toolkit to work, ArcInfo 9.3 must be in use on the user's computer, as this was the environment in which the toolkit was created. Toolkits can also be created for use in the ArcView and ArcEditor environments; however, this was not in the project scope. Minimum system requirements to run ArcInfo include a PC-Intel platform, with a Windows Vista, 2000, or XP operating system, 1 GB RAM memory, and a 1.6 GHz processor (Environmental Systems Research Institute [ESRI], n.d.). Visual Studio was chosen as the programming environment in which the functional components of the toolkit were created, due to familiarity with the Visual Basic programming language. However, the user does not need to have Microsoft Visual Studio or Visual Basic installed, as the necessary Visual Basic .NET components of the toolkit will be installed directly onto the user's computer from the toolkit's executable file. This feature makes the toolkit ideally portable, which was one of the requests of the client. There have also been discussions of making the toolkit available for download from the Tools and Scripts page of the USGS planetary GIS web server - PIGWAD (Planetary Interactive GIS-on-the-Web Analyzable Database) at http://webgis.wr.usgs.gov/ . The toolkit will then be available to any person who may be interested in its automated functions. The client also requested a simple graphical user interface for the toolkit, designed for users with only a basic familiarity with ArcGIS and the PPR text files to be processed. Accordingly, the user interface should display clear, concise instructions and require minimal input from the user. 
The operational requirements include the client-requested performance guidelines for the toolkit. The toolkit processing must be quick and efficient, as it is one of the primary objectives of the project. Its automated functions must not only be able to quickly generate new features and symbology, but should also be able to assign the Io_2000 geographic coordinate system to all sets of features and perform data clean-up functions. The toolkit should also notify the user as to any errors in the file paths entered into the user interface, so that these errors can be corrected by the user. A message box confirming the completion of processing is also helpful to the user as it signals that the toolkit is ready to process a new set of PPR text files.

The transitional requirements include all steps that need to be taken when handing over the toolkit to the client. A meeting was arranged to briefly train the client on how to use the toolkit. Along with the toolkit, a file geodatabase of newly processed features, and new ArcMap symbology, a detailed report was also necessary to serve as the help documentation for the toolkit. It will be the responsibility of the client to allocate space on a hard drive or network drive for the file geodatabase, the ArcMap document containing the new symbology, and the PPR text files. Likewise, updates and maintenance of the toolkit, after delivery to the client, is out of the defined project scope and thus will be the client's responsibility.

\subsection{System Design}

The functional and non-functional requirements determined the system design components for this project (Figure 3-1).

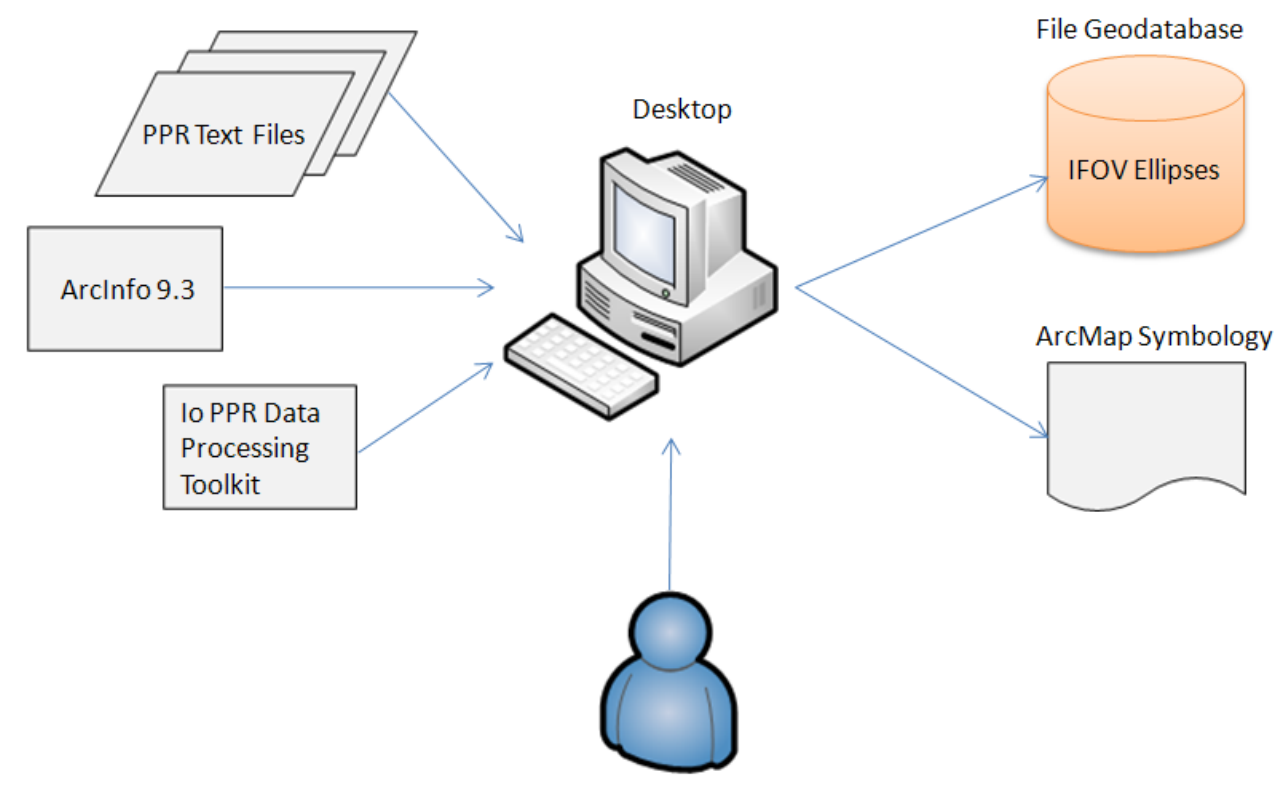

Figure 3-1: System architecture. 
The system was built for a desktop computing environment, with ArcInfo 9.3 installed. ArcInfo 9.3 is currently installed on the client's computer and was chosen, in consultation with the client, as the ArcGIS environment in which to build the new custom toolkit. Once this toolkit is installed, the user can process the raw data from the PPR text files through the simple, yet instructive, user interface of the ArcGIS Io PPR Data Processing Toolkit. New IFOV ellipse features are created and saved to a file geodatabase. An IFOV ellipse represents the area scanned on the ground surface of Io, at a particular moment in time, by the Photopolarimeter-Radiometer instrument. A file geodatabase was chosen for storage of the features, as it has virtually no size limitation (see Section 4.2 for details). The toolkit also assigns Io_2000 as the geographic coordinate system for these features and performs data clean-up functions (see Section 4.4). Additionally, the toolkit creates new symbology for the IFOV ellipse layers in ArcMap. The symbology is color-coded to match the spectrum of surface temperature values associated with the ellipses.

\subsection{Project Plan}

A project plan was then developed to manage the completion of key project tasks. The original project plan, written in December 2008, consisted of six major tasks/project phases, with an associated estimate of the number of hours needed to complete each phase:

- $\quad$ Phase 1: Requirements Analysis \& Development (40 hours)

- Phase 2: Data Download \& Exploration (20 hours)

- Phase 3: Project \& Register Data (160 hours)

- $\quad$ Phase 4: Geodatabase Design \& Implementation (60 hours)

- $\quad$ Phase 5: Prototype Development \& Testing (160 hours)

- Phase 6: ESRI User Conference, MIP Defense, MIP Report, \& Documentation (160 hrs)

As the project evolved, the project plan was adjusted. Originally, the goal of this project was to develop three separate toolkits to efficiently process three different types of data captured by the Galileo spacecraft's Photopolarimeter-Radiometer (PPR), NearInfrared Mapping Spectrometer (NIMS), and Solid State Imager (SSI), respectively. However, due to the one-year time constraint for this project, the scope was adjusted to encompass the completion of a toolkit for just one of these datasets, the PPR data. As a result, the first change in the project plan was the elimination of Phase 3, Project $\&$ Register Data. Processing of the PPR data files did not involve georeferencing of data layers, as was required with the NIMS and SSI imagery. The PPR temperature data is linked to specific coordinate points listed in the original data files. Initial plotting and 
projecting of these coordinate points then became a part of Phase 2, Data Download \& Exploration.

The second change in the project plan was the increase of hours needed to complete the latter project phases. Phase 5, Prototype Development \& Testing, took approximately 240 hours to complete instead of the originally planned 160 hours. This was due to challenges in finding and integrating programming methods to code for certain tool functions (see Section 6.4 for more details). Likewise, completing Phase 6, ESRI User Conference, MIP Defense, MIP Report, \& Documentation, took longer than expected approximately 460 hours versus the originally planned 160 hours. The increase in the number of hours was due to the complexity in writing about planetary GIS topics, spherical geometry equations, and particular programming methods used. Despite these setbacks, all fixed deadlines were met, including delivery of the prototype toolkit to the client in July 2009 and participation in the 2009 ESRI User Conference paper presentation sessions and Map Gallery.

Below are the detailed tasks involved in phases 1, 2, 4, 5, and 6:

1. In Phase 1, Requirements Analysis \& Development, meetings were conducted with the client, to determine the functional and non-functional requirements of the project, and a project plan was created.

2. In Phase 2, Data Download \& Exploration, the PPR data files for Io's Pele-Pillan region were downloaded and various files were gathered to provide reference for the data. The reference files included a background image of Io's surface, a latitude-longitude grid, and surface feature annotation for Io. The PPR data files were converted to Excel files and manually added to ArcMap; coordinate points were plotted and projected, and the data was explored within ArcMap.

3. In Phase 4, Geodatabase Design \& Implementation, the conceptual data design model and the physical geodatabase design model were created. Later in the project, the file geodatabase feature classes were created by using the completed Io PPR Data Processing toolkit. These feature classes were then stored in feature datasets based on orbit.

4. In Phase 5, Prototype Development \& Testing, an ArcGIS toolkit was developed and tested to process the original PPR data files. The criteria for the toolkit functions included automated creation of: 1) new IFOV ellipse features, 2) attribute tables containing the important temperature data, and 3) new temperature-specific symbology for the ellipse layers.

5. Phase 6 sums up the completion of the project with its title - ESRI User Conference, MIP Defense, MIP Report, \& Documentation. In this phase, a 
project presentation was given at the 2009 ESRI conference, along with submittal of a project poster for display. The Major Individual Project (MIP) defense and final report were also successfully completed. Lastly, all MIP files and documentation were sent to the client and the University of Redlands.

The complete, updated project plan is located in Appendix A and no longer includes the Project \& Register Data phase, as previously mentioned.

\subsection{Summary}

The overall system design and planning steps were discussed in this chapter. A requirements analysis was conducted to determine: 1 ) the functional qualities that the custom toolkit would need to possess and 2) the non-functional constraints on the proposed software system. The non-functional constraints include technical requirements imposed on the system by both the user and technology, as well as user-imposed operational and transitional requirements. The conceptual system design, incorporating these requirements, includes an ArcInfo 9.3 desktop computing environment that uses the Io PPR Data Processing Toolkit to: 1 ) process data from the PPR text files into IFOV ellipse file geodatabase feature classes, and 2) create new ArcMap symbology for the IFOV ellipse layers. A project plan was also developed, outlining the major project tasks and budgeted hours. Changes to the project plan were inevitable, but did not affect fixed deadlines. 


\section{Chapter 4 - Database Design}

Every GIS consists of a spatial database of geographic feature and attribute information. The design of a GIS database is important because it helps to identify the data that are necessary for the project, explores data values, relationships, and linkages, and organizes data as part of a workflow. The database design also helps in identifying potential data problems and incorporates the functional needs of the database users. This chapter describes the conceptual and logical data design models developed, the data sources used, data download procedures, important metadata, and data clean-up issues.

\subsection{Conceptual Data Model}

The conceptual data model comprises the entities of interest that address the project problem, and their relationships. This design model provides a structured layout of the big picture components of the project, which facilitates data organization and determination of a workflow. Essential tasks involved in creating a conceptual model include:

- identification of data objects or entities

- investigation of the corresponding attributes

- research into and identification of the data linkages that are essential for the final GIS output

The conceptual model for this project pulls together the requisite data for accurate plotting and representation of scanned areas on Io's surface, as well as the corresponding temperature values.

Unified Modeling Language (UML) diagrams were created in Visio to formally organize the conceptual design process. UML offers a standard scheme for the drafting and development of object-oriented software. There are four main UML shapes used in diagramming a conceptual data model for a GIS (Figure 4-1). The shapes symbolize:

1. Class - represented by a rectangle labeled with the data class name; attributes are also often listed.

2. Generalization - an unfilled arrow, which signifies a "type of/is a" relationship between two classes. (In Figure 4-1, a house is a type of building.)

3. Binary association - a line that connects two related classes; the numerical relationship between these classes is sometimes stated. (In Figure 4-1, a house contains many doors.) 
4. Composition - a diamond and line(s) connecting subclasses to a superclass. The diamond is positioned closest to the superclass. (In Figure 4-1, a house is composed of a ceiling, walls, and floors.) Sometimes, the numerical relationship between these classes is stated.

1. Class

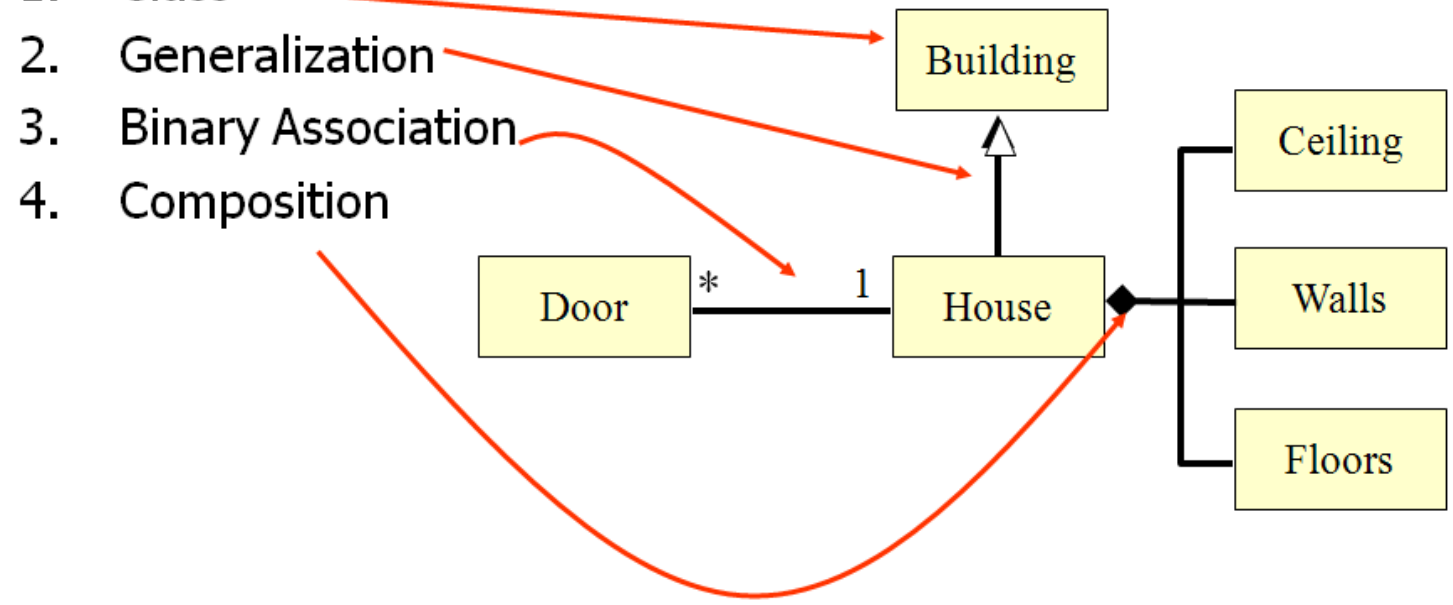

\section{Figure 4-1: The four principal UML shapes used in information system conceptual modeling.}

The conceptual model to accurately plot Photopolarimeter-Radiometer (PPR) surface temperature data for Jupiter's moon Io is simple in design (Figure 4-2). In processing the PPR data for Io's Pele-Pillan region, specific files containing the Temperature and Existing Geometry classes are chosen. The Temperature class consists of temperature values pertaining to the scans of Io's surface, while the Existing Geometry class contains positional information related to Io's surface scans and the Galileo spacecraft that carried the PPR instrument. The Temperature and Existing Geometry data are linked by an ObjectID. All Geometry is a superclass which contains the Existing Geometry and New Geometry subclasses. The New Geometry fields are the elliptic parameters of the Io surface scans and are calculated based on a variety of fields in the Existing Geometry class. For example, the Semi Major Axis field in the New Geometry class is calculated using values from the following fields of the Existing Geometry class: Latitude (of Field of View (FOV) intercept point), Longitude (of FOV intercept point), SSCLat (subspacecraft latitude), SSCLon (sub-spacecraft longitude), and SIPDIS (distance from spacecraft to FOV intercept point). The data in the All Geometry class are joined with the data in the Temperature class to create the class called PPR Scan for Pele-Pillan Region. Each PPR Scan contains many rows of Temperature and All Geometry data. In addition, each Temperature value is linked with one set of All Geometry data. The PPR Scans can then be categorized by orbit; there are four orbits that the spacecraft Galileo 
traversed in obtaining PPR data for Io's Pele-Pillan Region. Each orbit obtained data for multiple PPR Scans.

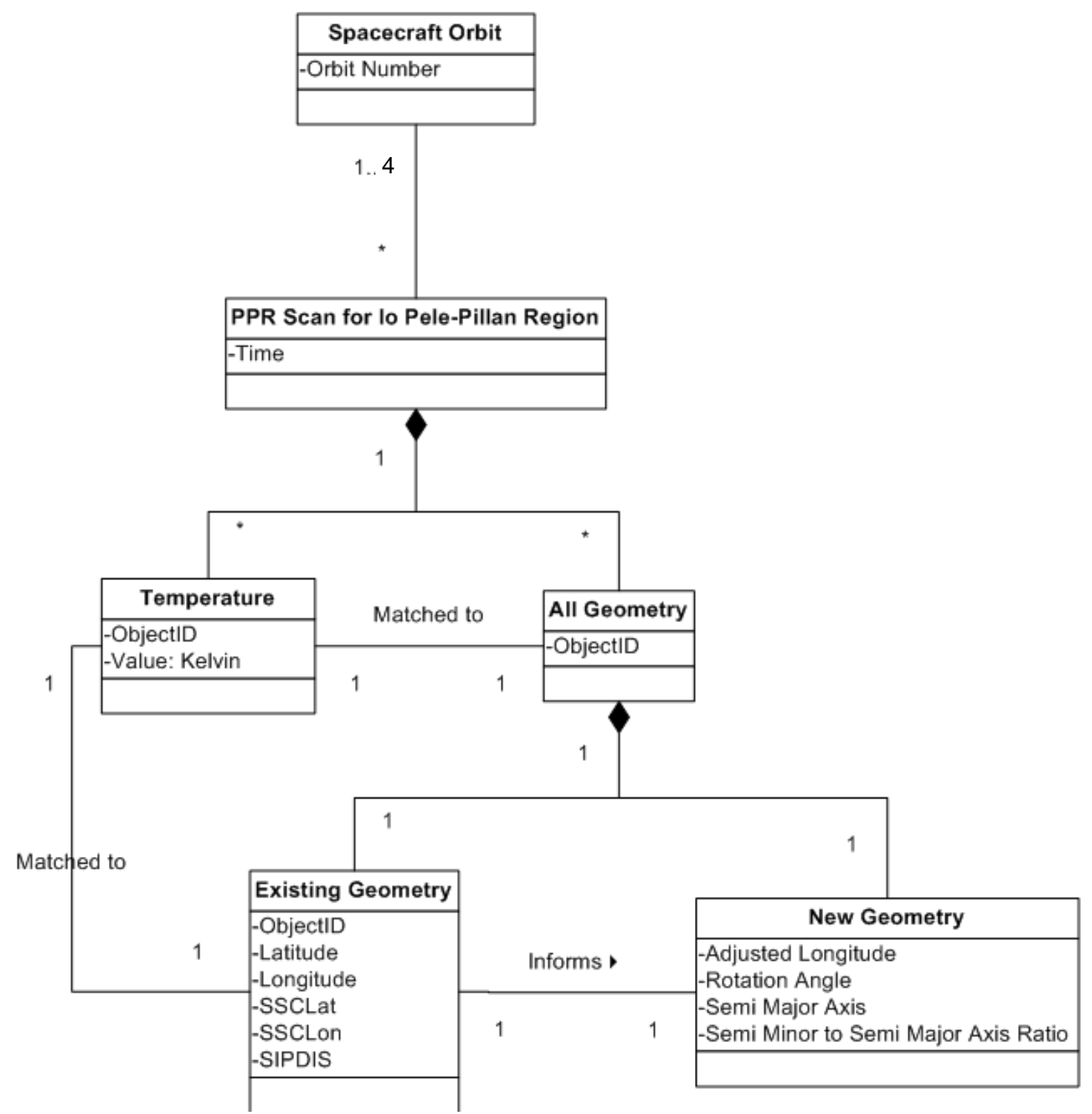

Figure 4-2: UML relationship diagram for PPR data.

\subsection{Logical Data Model}

The logical data model takes the conceptual model and moves it into the physical geodatabase design phase. A geodatabase was used in this project mainly for organization purposes and data integrity, providing a central location for easy data access and management. A geodatabase often contains multiple feature classes and the feature classes that have similar characteristics are frequently grouped together in feature datasets within the geodatabase. A geodatabase is also needed for single user and small 
workgroup scenarios, supporting concurrent use by a single data editor and many data viewers. Eventually, a geodatabase containing feature classes of the processed PPR scan data will be available for download from the USGS planetary GIS web server PIGWAD (Planetary Interactive GIS-on-the-Web Analyzable Database) at http://webgis.wr.usgs.gov/ .

An ArcGIS file geodatabase was chosen for the storage of PPR data feature classes, as it has virtually no size limitation. Each feature dataset, stand-alone feature class, or individual table can be up to one terabyte in size. Additionally, there is no overall size limit on the geodatabase; many feature datasets, stand-alone feature classes, and tables can be stored in each geodatabase. In comparison, an ArcGIS personal geodatabase has a size limit of just two gigabytes per database, with an effective limit between 250 and 500 megabytes before performance degrades (ESRI, 2008). The large size allowances for the file geodatabase provide the client and the planetary GIS community with the ability to add more Io datasets to the database. The compact data structure of the file geodatabase also allows for optimal performance. ESRI (2008) states that "file geodatabases use about one third of the feature geometry storage required by shapefiles and personal geodatabases”.

ArcGIS Diagrammer was used to model the data structure of the PPR file geodatabase (Figure 4-3). The data from the original PPR text files and the new polygon geometry calculations were programmatically saved together as geodatabase feature classes, with filenames representative of the original text files. These feature classes were organized into feature datasets based on orbit, which is the only relationship classification that can be made between the feature classes. Similarly, the original PPR data files were organized by orbit folders for download from the internet (see Section 4.3). The data did not have any sophisticated behavior or advanced geospatial rules, e.g., topologies and networks, to be represented in the logical data model. 


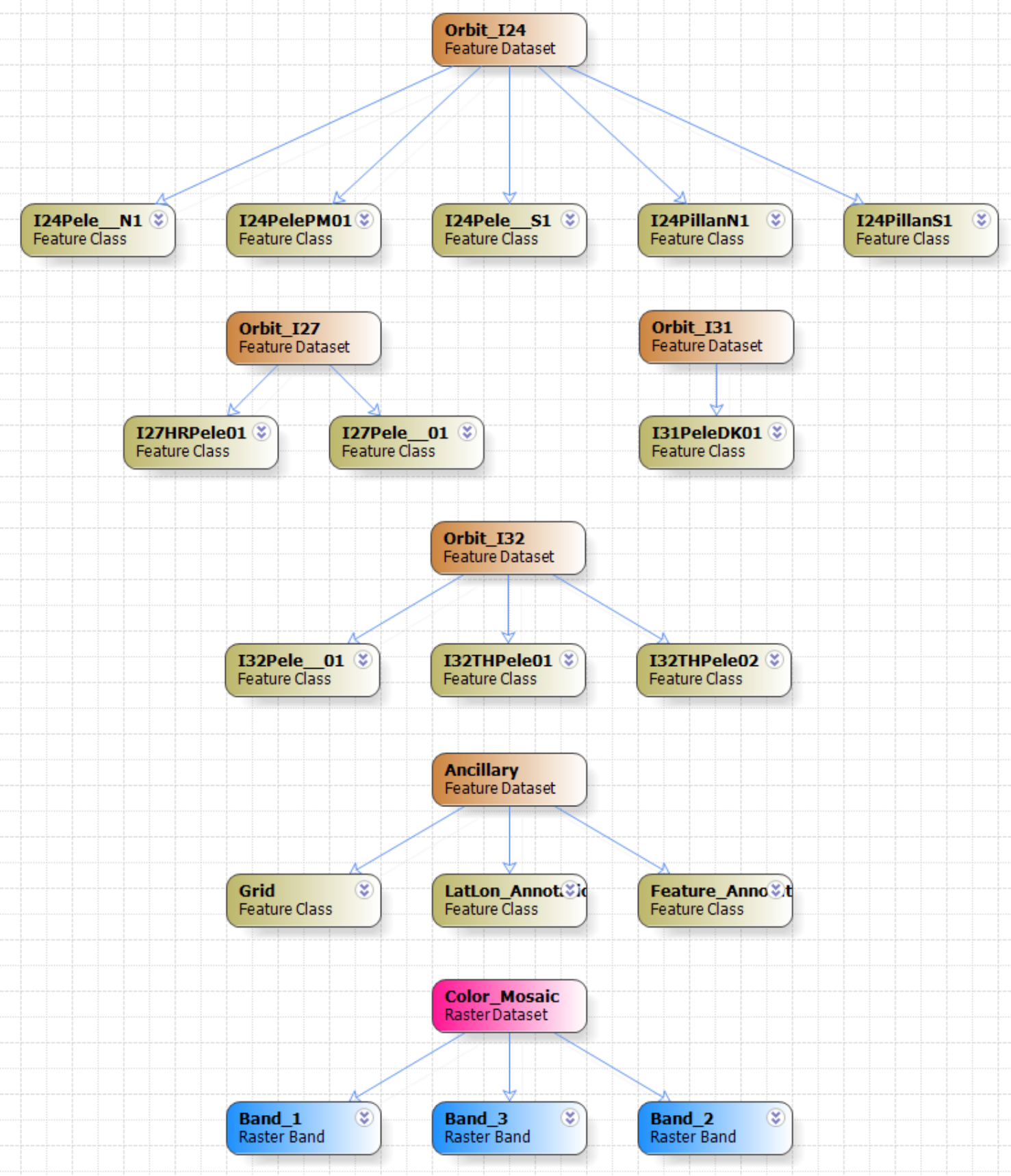

Figure 4-3: Logical model for PPR file geodatabase.

There are only a few differences between the conceptual model and the logical model. The logical model represents just the two classes displayed at the top of the conceptual model (Figure 4-2), Spacecraft Orbit and PPR Scan for Io Pele-Pillan Region. The data in the Temperature and All Geometry classes were programmatically added as attributes to the feature classes representing PPR Scans for Io Pele-Pillan Region; i.e. separate Temperature and All Geometry feature classes do not exist. The logical model 
also contains two additional datasets not found in the conceptual model, but which are useful for reference purposes: the Color_Mosaic raster dataset and the Ancillary feature dataset (Figure 4-3). The Color_Mosaic raster dataset is a global color mosaic of Io's surface and includes three bands: red, green, and blue. The Ancillary feature dataset contains a grid of $5^{\circ} \times 5^{\circ}$ squares and two annotation files, one for latitude-longitude reference and one for feature identification on Io's surface.

In order to make sure that all feature classes shared the same spatial reference, Io 2000 was specified as the geographic coordinate system for each new dataset. In ArcCatalog, the Io 2000 geographic coordinate system can be found in the Coordinate Systems folder, under Geographic Coordinate Systems $\rightarrow$ Solar System. It is the only coordinate system for Io found in ArcGIS 9.3. The specific parameters of the Io 2000 geographic coordinate system are as follows:

- Datum: D_Io_2000

- Spheroid: Io_2000_IAU_IAG

- Prime Meridian: Reference_Meridian

- Angular Unit: Degree

\subsection{Data Sources}

During the Galileo spacecraft mission, the PPR instrument captured data for many scanned segments of Io. Eleven of these scanned segments cover all or a portion of the Pele-Pillan region of Jupiter's moon Io (J. Rathbun, personal communication, November 17, 2008). The data for this project consist of a geometry file and a raw data record (rdr) file for each of these 11 scanned segments. All original data files are in text file format.

All PPR data can be downloaded from the following NASA Planetary Data System (PDS) webpage: http://pds-atmospheres.nmsu.edu/cgi-bin/getdir.pl?volume=gopr_5002 . The corresponding geometry and rdr files have the same filenames, but are stored in different subdirectories. To download the geometry files, click on the geometry subdirectory. Then click on the geo subdirectory. Next, indicate the orbit during which the scans were made and then indicate the observed body, which in this case is Io. Finally, download the geometry files. For Io's Pele-Pillan region, the following data scan segments were downloaded (as per the client, Dr. Rathbun):

- From orbit I24: PELE_N1, PELE_S1, PELEPM01, PILLANN1, PILLANS1

- From orbit I27: HRPELE01, PELE_01

- From orbit I31: PELEDK01

- From orbit I32: PELE_01, THPELE01, THPELE02

An overview of the process is shown in Figure 4-4. 


\begin{tabular}{|c|c|c|}
\hline $\begin{array}{l}\text { VOLUMES } \\
\text { rgopr_5002 }\end{array}$ & $\begin{array}{l}\text { Directories } \\
\text { and files for } \\
\text { gopr_5002 } \\
\text {-calib } \\
\text {-catalog } \\
\text {-document } \\
\text {-geometry } \\
\text {-index } \\
\text {-label } \\
\text {-r_edr } \\
\text {-rdr } \\
\text { aareadme.txt } \\
\text { errata.txt } \\
\text { voldesc.cat }\end{array}$ & $\begin{array}{l}\text { Contents of } \\
\text { calib } \\
\text { catalog } \\
\text { document } \\
\text { geometry } \\
\text { Index } \\
\text { label } \\
\text { r_edr } \\
\text { rdr } \\
\text { aareadme.txt } \\
\text { errata.txt } \\
\text { voldesc.cat }\end{array}$ \\
\hline
\end{tabular}

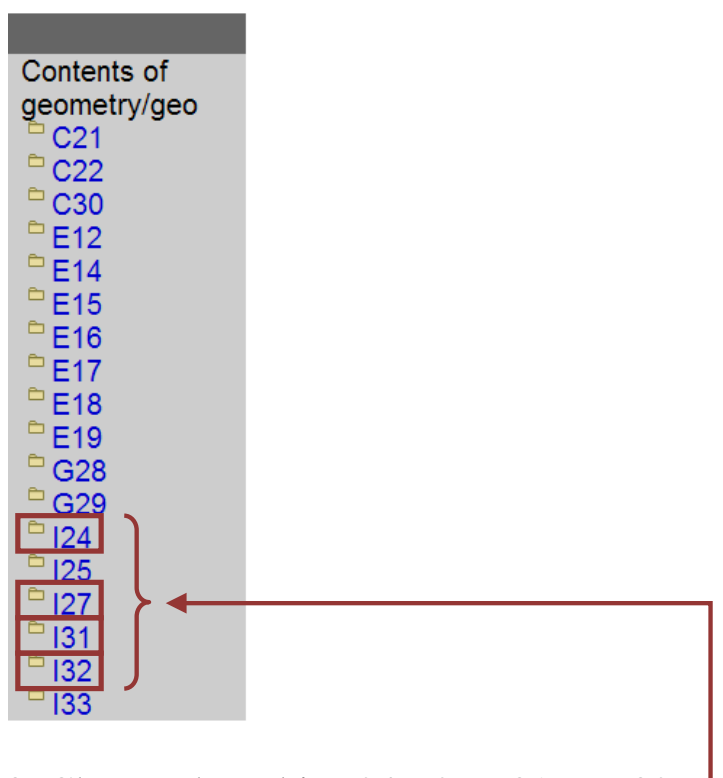

3. Choose the orbit, I24, I27, I31, or I32.

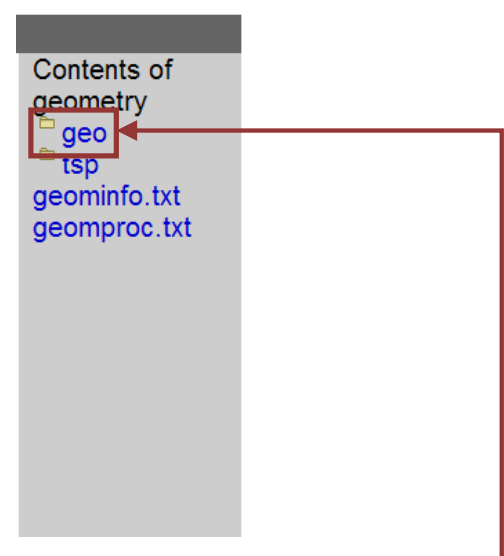

2. Next, click on the geo subdirectory.

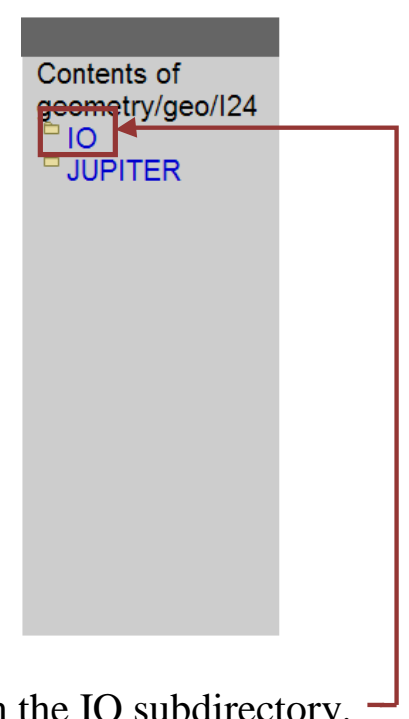

4. Click on the IO subdirectory.

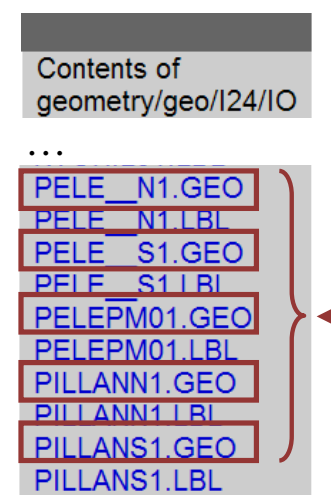

5. Download the appropriate .geo files for the associated orbit. The files shown are those that refer to the five scanned segments for the I24 orbit.

Figure 4-4: Geometry file download process. 
The rdr file download process is very similar to that just described for the geometry files. However, instead of clicking on the geometry and geo subdirectories, click on the rdr subdirectory. Then, similar to the geometry file download, choose the orbit, click on the IO subdirectory, and download the appropriate .tab files for the associated orbits. These files have the same names as the associated geometry files, but a different extension, .tab. Please note that for orbit I24, two rdr .tab files are labeled differently from the analogous geometry files. PELE_N01.TAB and PELE_S01.TAB should be downloaded to correspond to the PELE_N1 and PELE_S1 geometry files.

The data also need to be saved in a specific format for later incorporation into the geodatabase. In order to save the files in the rawest format, right click each file and choose the Save Target As... option. To facilitate proper organization, each file must be saved by specifying the associated orbit with the original file name from the NASA PDS website. For example, PELE_N1.GEO would be saved as I24PELE_N1.GEO. However, in the case of the PELE_N01.TAB and PELE_S01.TAB rdr files, they should be downloaded with the names I24PELE_N1.TAB and I24PELE_S1.TAB to match the appropriate geometry files. In total, there were 22 downloaded files, including 11 .geo files and 11 .tab files.

The metadata files contain additional information regarding the entire set of PPR data. These files can be obtained from http://starbrite.jpl.nasa.gov/pds/viewProfile.jsp?dsid=GO-J-PPR-3-RDR-V1.0 (Travis et al., 2002). Here, the nomenclature for orbit name is explained to be the letter corresponding to the targeted satellite and the orbit number, e.g., I24 has observations targeted at Io and is the $24^{\text {th }}$ orbit. Descriptions of the data fields are also given. The key fields used in this project and their descriptions are:

- ADJUSTED SCLK: Time of sample

- LAT: Latitude of Field of View (FOV) intercept point

- LON: Longitude of FOV intercept point

- SIPDIS: Distance from spacecraft to FOV intercept point, km

- SSCLON: Longitude of sub-spacecraft point

- SSCLAT: Latitude of sub-spacecraft point

- BRIGHTNESS TEMPERATURE: Apparent brightness temperature converted from the net data number value of scene radiance. When the noise and/or uncertainty of the data reduction is such that a negative value for the RADIOMETRY_DATA_NUMBER occurs, the conversion to the brightness temperature is performed as though the radiance value were positive and the corresponding brightness temperature is expressed then as a negative value. For non-radiometry PPR measurements, the brightness temperature value is set at 0000.0. 
Of further note, the brightness temperature has been corrected for instrument bias (Travis, et al., 2002), as outlined below:

Data reduction of the thermal radiometry entails the correction of the raw pyroelectric detector output for contributions to the measured flux by emission, albeit small, from internal elements such as mirrors and radiometric stops. These corrections use the element temperatures monitored by the thermistors along with calibration factors determined through ground thermal vacuum tests in which each element was in turn heated by several degrees to observe the sensitivity to that particular element.

The brightness temperature, however, is also affected by noise, caused by variable high-energy radiation (presumably particles) impacting the detector (Travis, et al., 2002). Generally, there is increased noise with decreasing distance of the spacecraft from Jupiter. Travis et al. (2002) state "noise remains a problem in many cases and one should use caution with respect to both the absolute accuracy and the precision of the PPR radiometry data."

Additional files, such as a global color mosaic of Io and a set of labels for 200 major surface features on Io (Figure 4-5), were obtained to provide a reference frame for the PPR data. The raster and annotation feature class were obtained from the Redlands Institute (Barrett, 2006b) and were originally derived, respectively, from the Gazetteer of Planetary Nomenclature (United States Geological Survey [USGS], 2009) and the Jupiter Satellites-Io web pages (USGS, 2008). There was no associated metadata for the Io surface feature labels. However, there was comprehensive metadata for the global color mosaic of Io (USGS, 2008) as follows:

This Galileo global false color mosaic was constructed from low phase angle violet, green, and near-infrared (756 nanometer) images from orbits G2, E6, C9, and C21. The images were calibrated using the best end-of-mission calibration information, corrected empirically for limb-darkening, and map projected using the camerapointing corrections of Archinal et al. (2001). The coregistered color images were next hand-edited to remove topographic shadows and pixels too near the limb, and mosaiced using a numerical procedure (Soderblom et al., 1978) that reduces the mismatch at the seams. This mosaic represents our best understanding of Io's color as pictured during the Galileo Mission. The true colors that would be visible to the eye are similar but much more muted than shown here. The spatial resolution of the mosaic ranges from 1.3 to $21 \mathrm{~km} /$ pixel at the equator, with the poorest resolution on the Jupiter-facing hemisphere of Io. 


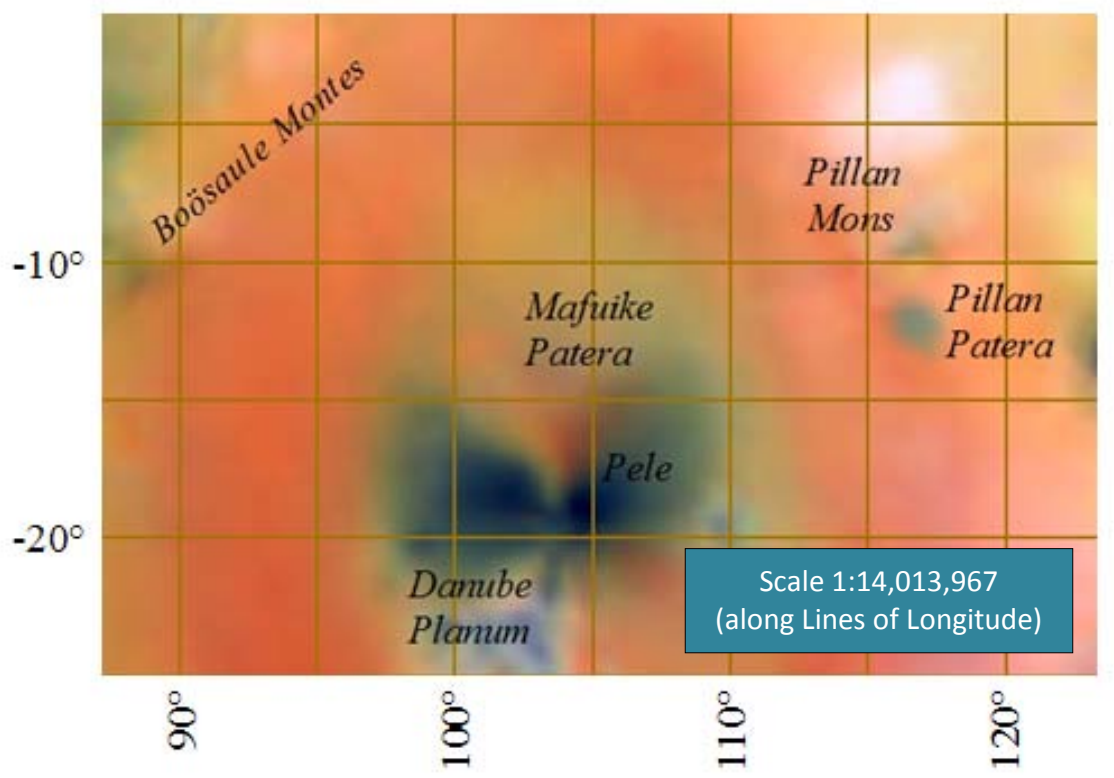

Figure 4-5: Subset of Io's surface feature annotation.

The colors in the Galileo global false color mosaic (Figures 4-5 and 4-6) indicate the composition of materials on the surface of Io (Davies, 2007). Most volcanic plume deposits on Io appear as white, yellow, or red - colors typical of sulfur. Volcanic pits and new deposits from eruptions, however, appear gray or black in color, revealing a greater concentration of silicates. Figure 4-5 provides a close-up view of Io's Pele-Pillan region, showing the gray silicate regions of the Pele and Pillan volcanoes and fresh lava/pyroclastic material deposits from their eruptions. The red ring of sulfur that was deposited by Pele's plume is also apparent.

\subsection{Data Scrubbing and Loading}

This project is about the processing of data in the PPR text files for incorporation into ArcGIS. As such, a substantial portion of the project consisted of the preparation of data for loading into geodatabase files. The process is outlined in greater detail in Chapter 5 , which describes the implementation phase of the project. This section will briefly introduce the issues addressed in clean-up of the data: longitude value adjustments, invalid data records, and null or negative temperature values.

When the latitude and longitude values were plotted from the PPR data files (spanning the Pele-Pillan region) onto the surface of Io, the points were nowhere near the red Pele plume deposit that is located in the eastern portion of Io's southern hemisphere, as revealed in Figure 4-6. It appears that the Io geographic coordinate system in ArcGIS uses East longitude (numbers increase to the east of the center longitude from 0 to $180^{\circ}$ and decrease to the west of the center longitude from 0 to $-180^{\circ}$ ) (Figure 4-6), while all 
NASA data use a coordinate system with West longitude (numbers increase to the west of the center longitude from 0 to $360^{\circ}$ ) (J. Rathbun, personal communication, February 16, 2009). The latitude and longitude parameters for both sets of coordinate systems are listed in Table 3.

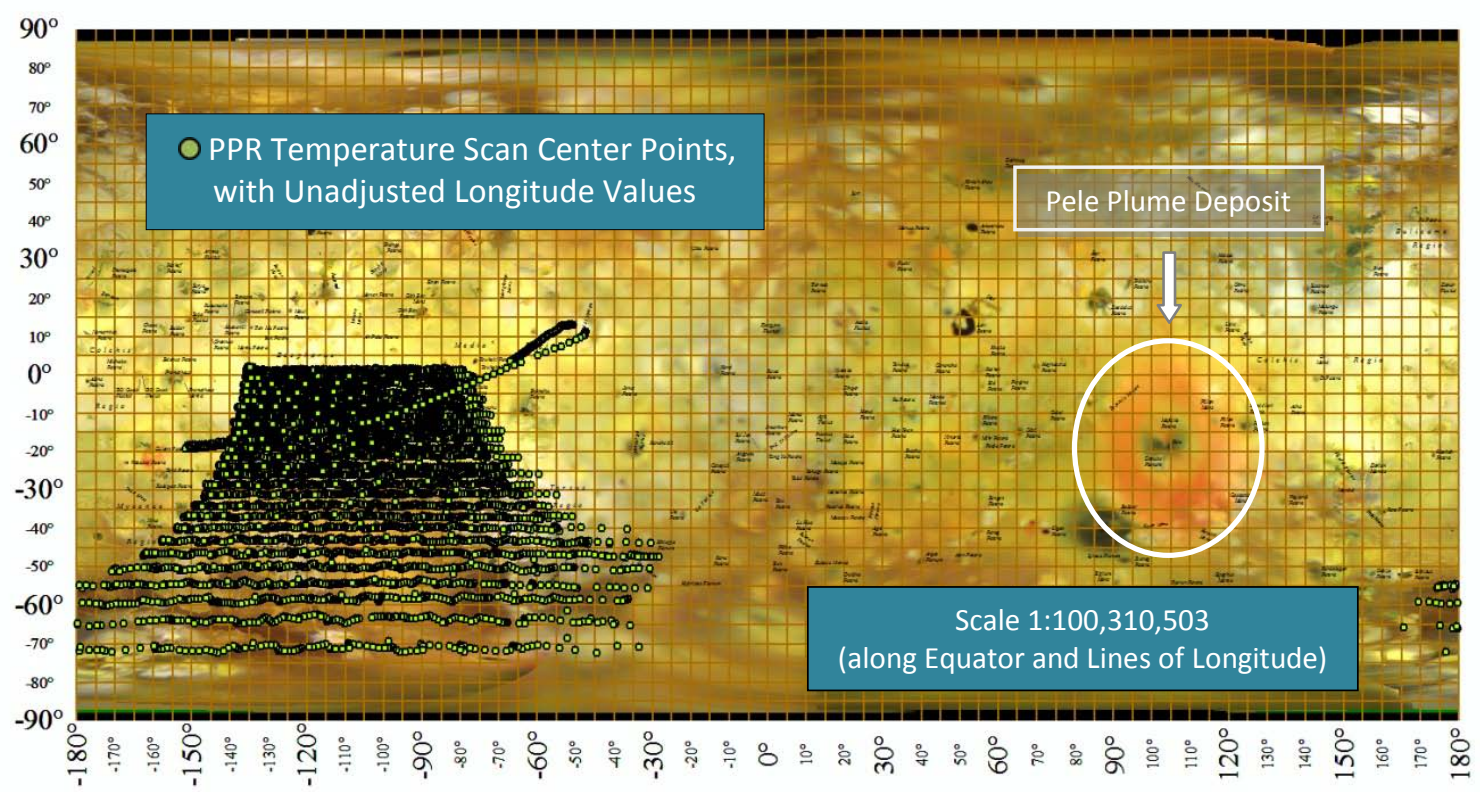

Figure 4-6: Initial plotting of data points in ArcGIS Io Geographic Coordinate System.

Table 3. Latitude and longitude parameters for Io Geographic Coordinate Systems (ArcGIS and NASA).

\begin{tabular}{|c|c|c|}
\hline \multicolumn{3}{|c|}{ Io Geographic Coordinate System } \\
\hline Software & ArcGIS & NASA \\
\hline Latitude Range & -90 to $90^{\circ}$ & -90 to $90^{\circ}$ \\
\hline Longitude Range & -180 to $180^{\circ}$ & 0 to $360^{\circ}$ \\
\hline Center Longitude & $0^{\circ}$ & $0^{\circ}$ \\
\hline
\end{tabular}

To adjust for the discrepancy in values between the two coordinate systems, all longitude values $>180$ in the data were plotted as 360 minus longitude in the ArcGIS Io geographic coordinate system, and all longitude values $<180$ in the data were plotted as negative longitude in the ArcGIS Io geographic coordinate system. This rule was also 
applied to the sub-spacecraft longitude (SSCLON) values. The newly plotted data points correctly appear in the eastern portion of Io's southern hemisphere, over the red Pele plume deposit (Figure 4-7). In order to more accurately visualize spherical distance and direction, from the mid-range of data points, Figure 4-8 shows all data projected using an azimuthal equidistant projection, with the latitude of origin at $-35^{\circ}$ and central meridian at $105^{\circ}$.

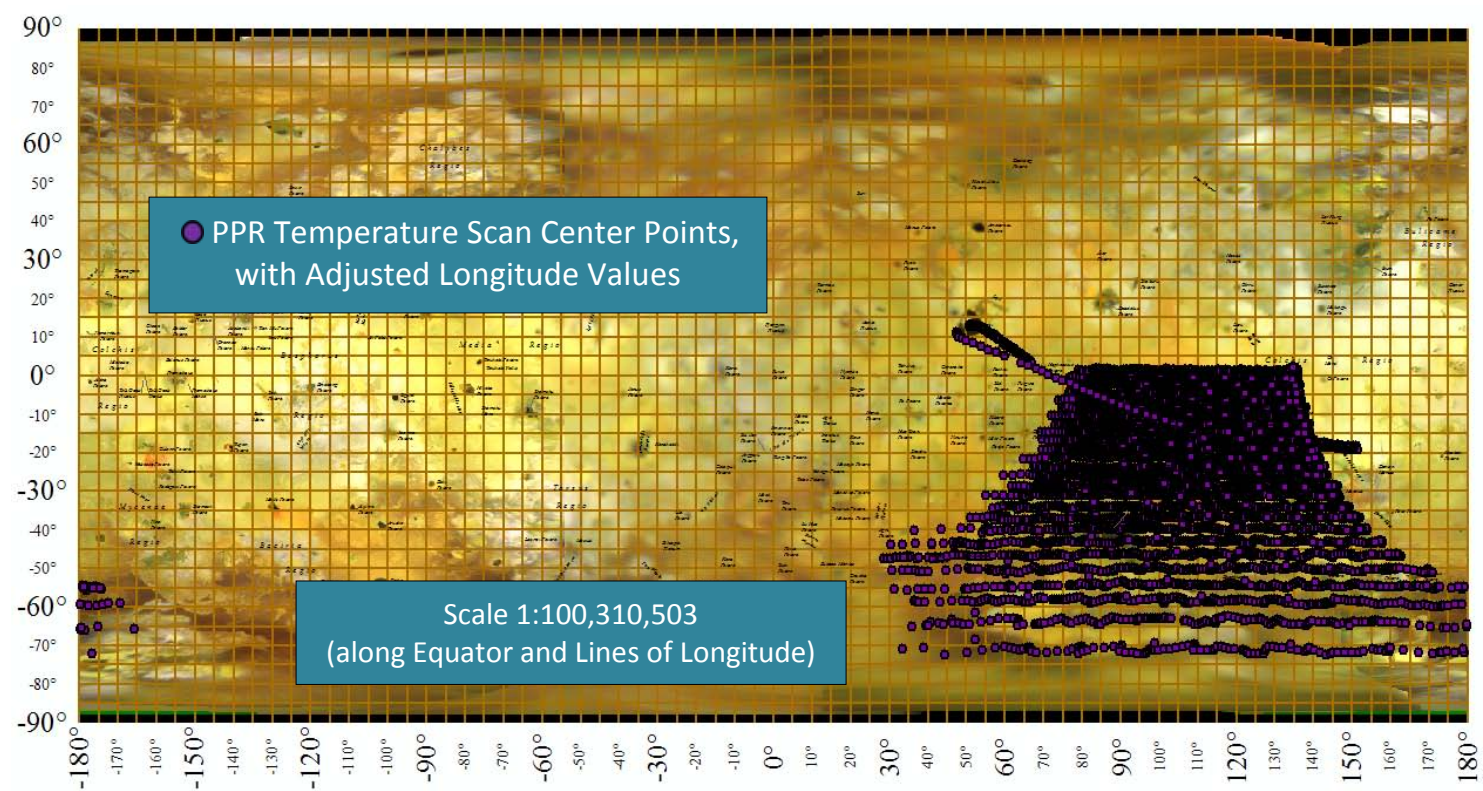

Figure 4-7: Plotting of data points, with adjusted longitude values, in ArcGIS Io Geographic Coordinate System.

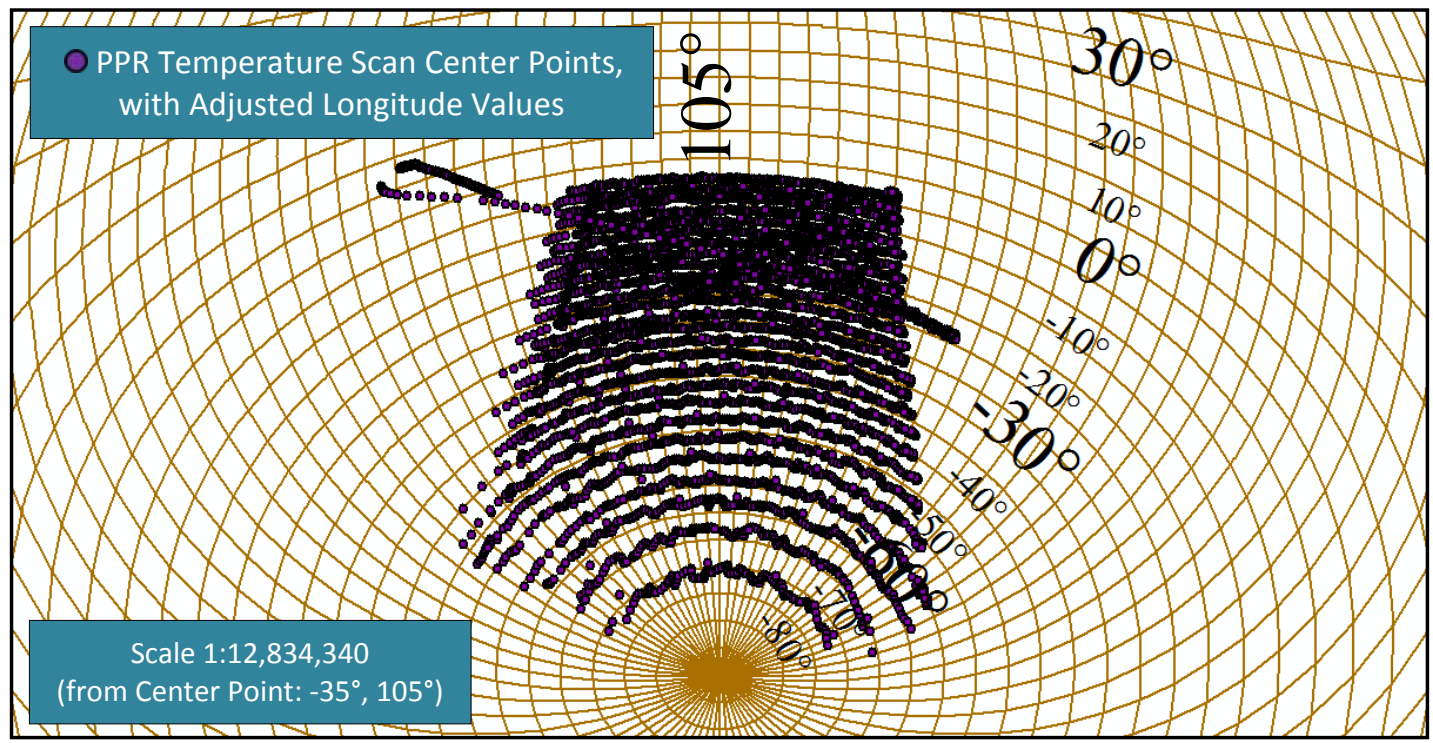

Figure 4-8: PPR data points plotted on surface of Io, using an azimuthal equidistant projection. 
The remaining data issues dealt with invalid records that were not to be included in the geodatabase files. Some records did not contain valid readings and were marked with 666 for various values, such as LAT and LON. Additionally, when no radiometric PPR measurements were taken, the temperature values were set at 0000.0, as mentioned previously. Some temperature values were found to be set at 0.0 , which was treated to be the same as a value of 0000.0. Also mentioned in the previous section, when there was a significant amount of noise or uncertainty affecting the scene radiance values, the temperature values were expressed as invalid negative numbers. The solution was to programmatically skip these data records when creating the geodatabase files.

\subsection{Summary}

In this chapter, the principles of what constitutes a good database design were discussed. A conceptual model, often represented as a UML diagram, was first constructed to plan for the necessary data and workflow of the project. The UML diagram for this project shows multiple PPR data scans for the four orbits covering the Pele-Pillan region of Io. Temperature readings and the associated geometry are combined to create the PPR data scans. A logical model was constructed next to plan the details of the PPR file geodatabase physical design. The logical model consists of feature classes for the PPR data scans, organized into datasets for the different orbits. To provide a frame of reference for the PPR data scans, a raster dataset of a color mosaic of Io and a feature dataset, consisting of a latitude-longitude grid and annotation, are also included in the logical model. The next steps involved acquisition of the different sets of data from the NASA PDS website and from the Redlands Institute. Metadata was also available and proved essential to understanding the data used in this project. Finally, issues such as longitude value adjustments, invalid data records, and null/negative temperature values had to be cleaned up for incorporation of the data into the geodatabase files. 



\section{Chapter 5 - Implementation}

This project aimed to build an automated workflow to process data, captured by the PPR instrument onboard the Galileo spacecraft and stored in text file format, into feature class formats for use in ArcGIS. To do so, the data needed to be transformed from their original coordinates to elliptic shapes that more realistically represent the instantaneous field of view (IFOV) captured by the PPR instrument at that particular moment in time. This chapter first introduces the calculations involved in transforming the coordinate points into IFOV ellipses. The custom built ArcGIS tools to create the new features are then discussed. The first tool implements the aforementioned calculations to construct IFOV ellipses and their center points, while the second tool classifies the IFOV ellipse layers based on temperature.

\subsection{Transformation of Coordinate Points into IFOV Ellipses}

The PPR instrument, onboard the Galileo spacecraft, recorded each temperature scan of Io's surface with a pair of geographic coordinates. However, the temperature value for each scan actually represents an average of surface temperatures for the elliptical area of Io captured within the PPR instrument's instantaneous field of view at that particular moment in time (J. Rathbun, personal communication, October 11, 2008; NASA, n.d.). Therefore, the elliptical areas scanned on the ground by the PPR instrument, instead of the coordinate points, should be used to more accurately map the surface temperatures on Io. For the remaining report, the elliptical areas are called IFOV ellipses and each represents the true specific area that corresponds to a temperature scan. The IFOV ellipse can be mathematically defined by the angle of view sensed by the PhotopolarimeterRadiometer and the sensor's location. Due to oblique viewing and the curvature of Io, the further away the Galileo spacecraft was from nadir (position directly above the area being scanned), the more elongated the surface scans of Io were as compared to a perfect circle.

In order to create IFOV ellipses in ArcGIS, custom programming is necessary because there are no required out-of-the-box tools available. Parts of existing code (Barrett, 2006a) were used in this project, employing the IEllipticArc interface of the ESRI ArcObjects Geometry library to draw IFOV ellipses. The ArcObjects libraries are sets of software programming components used to build and extend the entire ArcGIS suite. For context, the unique parameters necessary to create an ellipse using the IEllipticArc.PutCoordsByAngle method of the IEllipticArc interface must be briefly defined (Table 4). The center point is the specific point that the ellipse is centered on. The semi major axis and the minor-major ratio determine the full ellipse on which the elliptic arc is generated. Finally, the rotation angle is the angle at which the ellipse is 
rotated to match the angle between the line of latitude of the sub-spacecraft point and the line connecting the sub-spacecraft point to the ellipse center.

Table 4. Parameters necessary to create an ellipse.

\begin{tabular}{|l|l|}
\hline Eenter point & $\begin{array}{l}\text { Definition } \\
\text { Coordinates of a particular temperature scan from the } \\
\text { original data files (ellipse center point) }\end{array}$ \\
\hline or & $\begin{array}{l}\text { Smallest axis spanning between points on opposite } \\
\text { sides of the ellipse }\end{array}$ \\
\hline Semi minor axis & $\begin{array}{l}\text { Half of the minor axis } \\
\text { or }\end{array}$ \\
\hline Semi major axis & $\begin{array}{l}\text { Largest axis spanning between points on opposite } \\
\text { sides of the ellipse }\end{array}$ \\
\hline Minor-major ratio & $\begin{array}{l}\text { Half of the major axis } \\
\text { (or ratio of the semi minor axis to the semi major axis) }\end{array}$ \\
\hline Rotation angle & $\begin{array}{l}\text { Azimuth angle between: } \\
\text { 1) the line of latitude of the sub-spacecraft point and } \\
\text { 2) the line connecting the sub-spacecraft point to the } \\
\text { coordinates of a particular temperature scan (ellipse } \\
\text { center point) }\end{array}$ \\
\hline
\end{tabular}

However, the parameters and data inputs listed above cannot be obtained directly from the original PPR files. Instead, specific data from the original PPR files must be manipulated by applying transformation equations, in order to obtain values for the ellipse parameters listed above. For these calculations, the values from the following fields in the geometry data files were used: LAT, LON, SIPDIS, SSCLON, and SSCLAT (Figure 5-1). As mentioned in Section 4.3, the description of these fields is as follows:

- LAT: Latitude of Field of View (FOV) intercept point

- LON: Longitude of FOV intercept point

- SIPDIS: Distance from spacecraft to FOV intercept point, km

- SSCLON: Longitude of sub-spacecraft point

- SSCLAT: Latitude of sub-spacecraft point. 
The latitude and longitude of the sub-spacecraft point is the latitude and longitude of a point on the surface of Io, at which a line can be drawn vertically $90^{\circ}$ from the ground surface, intercepting the spacecraft.

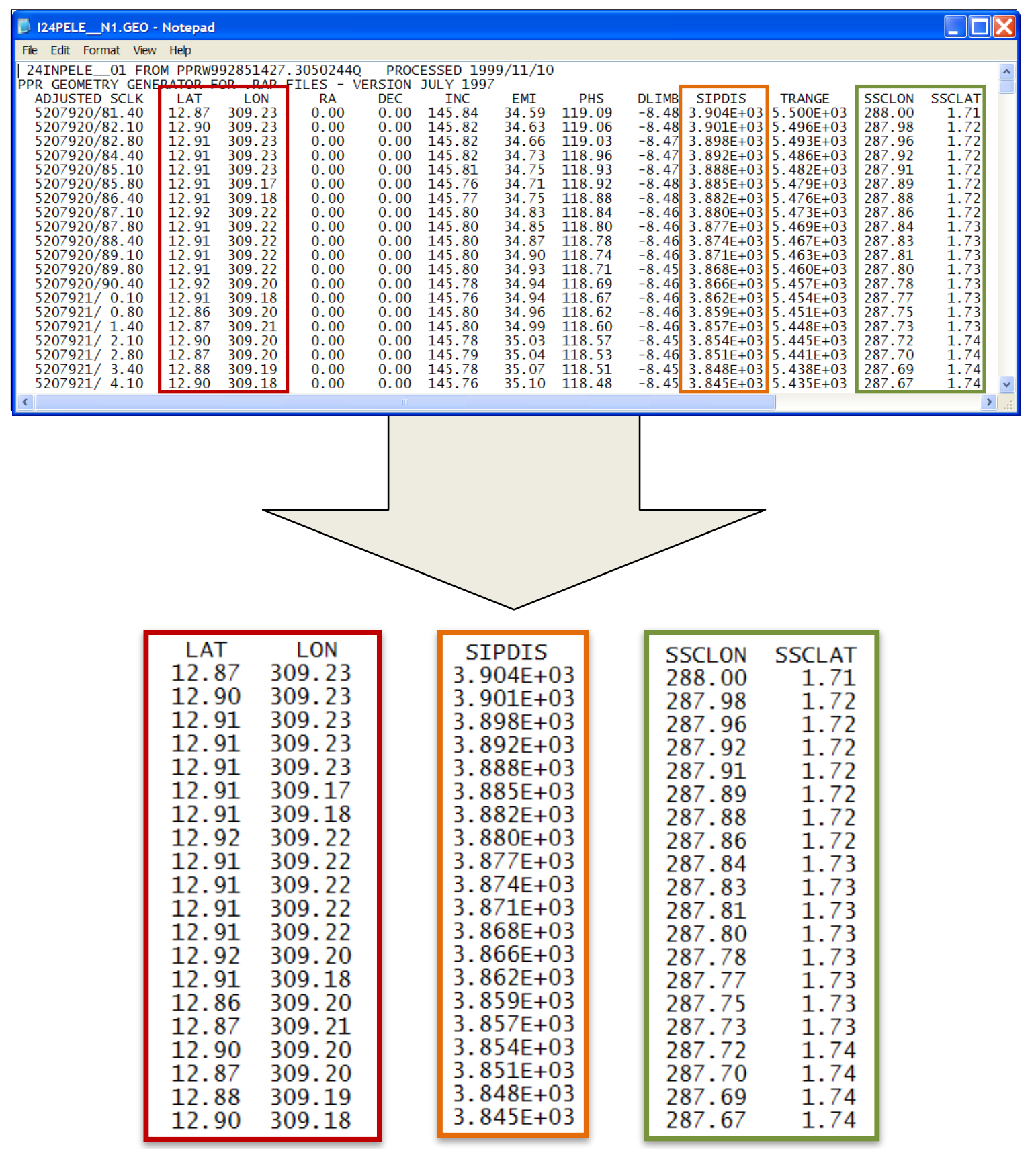

Figure 5-1: Field values in PPR geometry files used to calculate ellipse parameters.

The equation calculations were initially inserted into respective cells in an Excel spreadsheet. This was done particularly to test whether each calculated value in the sequence of spherical geometry equations was correct, as the values from some equations 
plug directly into other more complex equations. Referring back to previous cells is much easier, cleaner, and has less chance for error than creating one lengthy spherical geometry equation for the semi major axis, the minor-major ratio, and the rotation angle. Each ellipse center point required a simple transformation for longitude values.

The specific equations and factors used to calculate the ellipse parameters are described in the following sections.

\subsubsection{Coordinate System Conversion}

As mentioned in Section 4.4, values in both LON and SSCLON fields had to be adjusted in order to plot correctly in the Io geographic coordinate system in ArcGIS. All NASA data use a coordinate system with West longitude (numbers increase to the west of the center longitude from 0 to $360^{\circ}$ ), while the Io geographic coordinate system in ArcGIS uses East longitude (numbers increase to the east of the center longitude from 0 to $180^{\circ}$ and decrease to the west of the center longitude from 0 to $-180^{\circ}$ ) (Figure 4-6 and Table 3 in Section 4.4). To adjust for the difference, all longitude values $>180$ in the data were plotted as 360 minus longitude, and all longitude values $<180$ in the data were plotted as negative longitude (Equations 1 and 2). This resulted in two new calculated fields, NEWLON and NEWSSCLON, for the longitude of the FOV intercept point and the subspacecraft point respectively.

Equation 1. - Coordinate system conversion for longitude (LON) values.

$$
N E W L O N=\operatorname{IF}(L O N>180,360-L O N,-L O N)
$$

\section{Equation 2. - Coordinate system conversion for sub-spacecraft longitude (SSCLON) values.$$
N E W S S C L O N=\operatorname{IF}(S S C L O N>180,360-S S C L O N,-S S C L O N)
$$

\subsubsection{Photopolarimeter-Radiometer Instantaneous Field of View}

The PPR instrument onboard the Galileo spacecraft had an IFOV of 2.5 milliradians (Hansen J. E., n.d.). An IFOV is the cone-shaped angle of view, $\beta$, sensed by the detector (Aronoff, 2005) (Figure 5-2). The instrument's IFOV aids in determining the size of the elliptical areas scanned on the ground surface of Io. Consequently, the IFOV is used in calculations to determine the semi minor and semi major axes of these ellipses. 


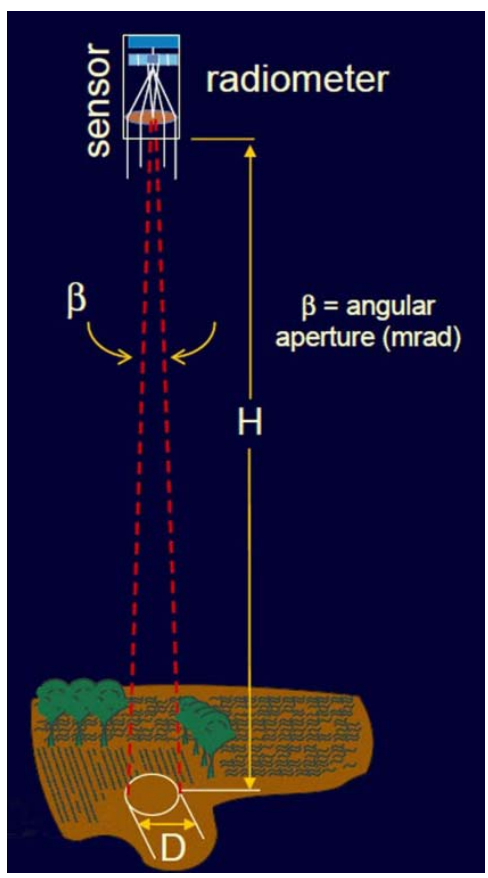

Figure 5-2: Instantaneous field of view of spacecraft instrument ${ }^{1}$.

\subsubsection{Radius of Io}

The radius of Io is approximately 1820 kilometers (Fischer, 2001). The radius of Io was used to convert the ellipse semi minor axis and semi major axis lengths from kilometers to arc degrees.

\subsubsection{Semi Minor Axis}

The semi minor axis is half of the minor axis, which is the smallest axis spanning between points on opposite sides of the ellipse (Figure 5-3). The semi minor axis is calculated based on the trigonometric function of the tangent of a right triangle (Figure 5-4). Here, $\theta$ represents half the PPR instrument's IFOV of 2.5 milliradians; $a$ represents the distance from the spacecraft to the FOV intercept point on the surface of Io; and $o$ represents the semi minor axis of the IFOV ellipse. In order to solve for the semi minor axis, $o$, the equation in Figure 5-4 can be converted to $o=a * \tan \theta$, where $a$ is the SIPDIS field value from the original PPR geometry data files and $\theta$ is measured in radians equaling $\left(\frac{2.5 \text { milliradians }}{2}\right) *\left(\frac{1 \text { radian }}{1000 \text { millira dians }}\right)$. The final equation for calculating the semi minor axis in kilometers is given in Equation 3.

\footnotetext{
${ }^{1}$ From Lectures in Remote Sensing: Optical Remote Sensing by J. Clevers, 2006, Centre of GeoInformation, Environmental Sciences, Wageningen University and Centre. Retrieved July 21, 2009, from http://www.geo-informatie.nl/courses/grs20306/course/Schedule/Optical-RS-new.pdf
} 


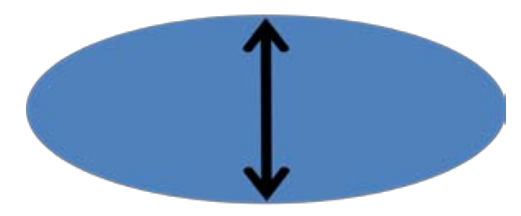

Figure 5-3: Minor axis of IFOV ellipse. The semi minor axis is half this length.
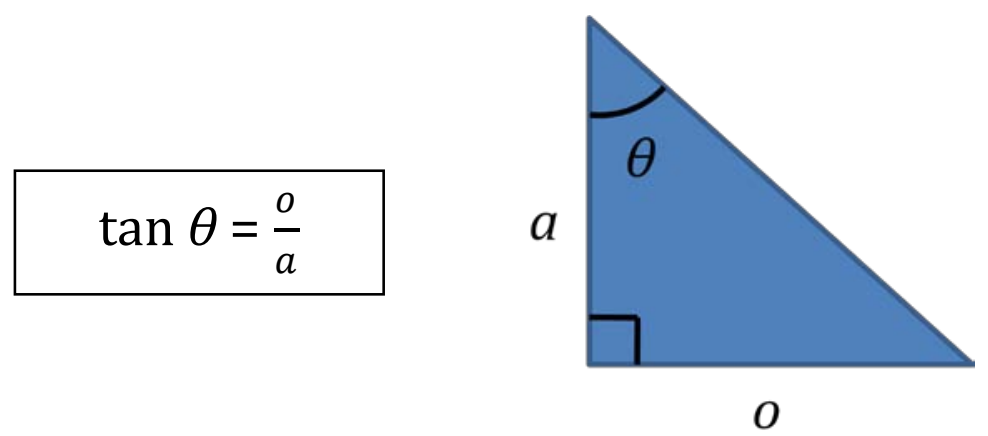

Figure 5-4: Tangent of a right triangle.

Equation 3. - Semi minor axis in kilometers (SMIN_KM).

$$
S M I N \_K M=\operatorname{SIPDIS} * \operatorname{TAN}\left(\frac{2.5}{2 * 1000}\right)
$$

However, in order to plot the IFOV ellipse in the Io Geographic Coordinate System in ArcGIS, the semi minor axis must be converted from kilometers to arc degrees (Equation 4) and then, arc degrees must be converted to decimal degrees (Equation 5).

Equation 4. - Semi minor axis in arc degrees (SMIN_AD).

$$
S M I N \_A D=\frac{S M I N \_K M * 180}{\pi * 1820}
$$

In Equation 4, the semi minor axis in arc degrees (SMIN_AD) is equal to the semi minor axis in kilometers (SMIN_KM) multiplied by the circumference of Io in degrees / the circumference of Io in kilometers; where the circumference of Io in degrees is 360, 
the circumference of Io in kilometers is equal to $2 * \pi *$ radius of Io, and the radius of Io is 1820 kilometers.

\section{Equation 5. - Semi minor axis in decimal degrees (SMIN_DD).}

$$
S M I N \_D D=\frac{S M I N \_A D}{\operatorname{COS}\left(L A T * \frac{\pi}{180}\right)}
$$

Equation 5 was provided by the client, Dr. Rathbun, to convert the semi minor axis from arc degrees to decimal degrees centered about the latitude of the ellipse center point. In Equation 5, the semi minor axis in decimal degrees (SMIN_DD) is equal to the semi minor axis in arc degrees (SMIN_AD) divided by the cosine of the latitude of the ellipse center point in radians. The latitude of the ellipse center point is the LAT field value from the original PPR geometry data files, multiplied by a factor of $\left(\frac{\pi}{180}\right)$ for conversion to radians.

\subsubsection{Semi Major Axis}

The semi major axis is half of the major axis, which is the largest axis spanning between points on opposite sides of the ellipse (Figure 5-5). Equation 6 was provided by the client to calculate the semi major axis in kilometers. This equation takes into account the semi minor axis in kilometers (SMIN_KM), the ellipse center point latitude and longitude values (LAT and NEWLON), and the sub-spacecraft point latitude and longitude values (SSCLAT and NEWSSCLON). In order to perform the calculation, the LAT, NEWLON, SSCLAT, and NEWSSCLON field values were converted to radians by multiplying by a factor of $\left(\frac{\pi}{180}\right)$.

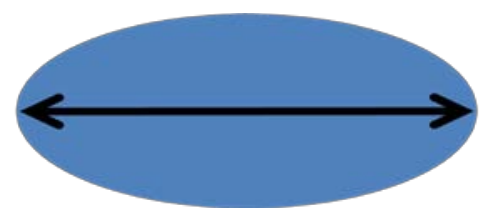

Figure 5-5: Major axis of IFOV ellipse. The semi major axis is half this length. 
Equation 6. - Semi major axis in kilometers (SMAJ_KM).

$$
S M A J_{-} K M=\frac{S M I N_{-} K M}{\left(\begin{array}{c}
\left(\operatorname{COS}\left(\operatorname{LAT} * \frac{\pi}{180}\right) * \operatorname{COS}\left(\operatorname{SSCLAT} * \frac{\pi}{180}\right) * \operatorname{COS}\left((\operatorname{NEWLON}-N E W S S C L O N) * \frac{\pi}{180}\right)\right) \\
+\left(\operatorname{SIN}\left(\operatorname{LAT} * \frac{\pi}{180}\right) * \operatorname{SIN}\left(\operatorname{SSCLAT} * \frac{\pi}{180}\right)\right)
\end{array}\right)}
$$

Similar to the semi minor axis calculation, the semi major axis must also be converted from kilometers to arc degrees (Equation 7) and then, arc degrees must be converted to decimal degrees (Equation 8).

Equation 7. - Semi major axis in arc degrees (SMAJ_AD).

$$
S M A J \_A D=\frac{S M A J_{-} K M * 180}{\pi * 1820}
$$

Equation 8. - Semi major axis in decimal degrees (SMAJ_DD).

$$
S M A J_{-} D D=\frac{S M A J_{-} A D}{\operatorname{COS}\left(L A T * \frac{\pi}{180}\right)}
$$

\subsubsection{Minor-major Ratio}

The minor-major ratio is the ratio of the minor axis to the major axis of the ellipse (Figure 5-6). Since the ellipse semi minor and semi major axes (SMIN_DD and SMAJ_DD) have already been calculated, these values are used in place of the minor and major axes to calculate the minor-major ratio (MINMAJ_RAT) in Equation 9.

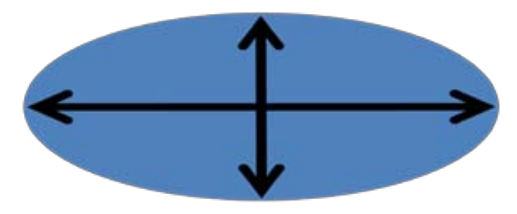

Figure 5-6: Minor-major ratio of IFOV ellipse.

Equation 9. - Semi minor axis to semi major axis ratio (MINMAJ_RAT).

$$
M I N M A J \_R A T=\frac{S M I N \_D D}{S M A J \_D D}
$$




\subsubsection{Rotation Angle}

The rotation angle represents the angle at which a ground surface area (IFOV ellipse) was scanned on Io in relation to the location of the spacecraft. The rotation angle in ArcGIS is the angle at which the ellipse is drawn, rotated from $0^{\circ}$ (Figure 5-7), representing the azimuth angle created from: 1) the line of latitude of the sub-spacecraft point and 2) the line connecting the sub-spacecraft point to the center point of a corresponding ellipse scanned on the surface of Io at that particular moment in time. Equation 10 calculates this azimuth angle, or ellipse rotation angle (ROT_ANGLE), in radians. The rotation angle must be in radian measurements as required by the IEllipticArc.PutCoordsByAngle method of the IEllipticArc interface in the ESRI ArcObjects Geometry library. Equation 10 was derived from an azimuth equation in Ghilani \& Wolf (2008). If the difference between the latitude of the ellipse center point (LAT) and the latitude of the subspacecraft point (SSCLAT) is 0 , the rotation angle is 0 . Otherwise, the rotation angle is calculated by subtracting the angle formed by arctangent $\left(\frac{\text { NEWLON }- \text { NEWSSCLON }}{\text { LAT - SSCLAT }}\right)$ from $\pi / 2$ $\left(90^{\circ}\right)$. The If...Then...Else statement in Equation 10 accounts for a denominator of 0 for LAT minus SSCLAT.

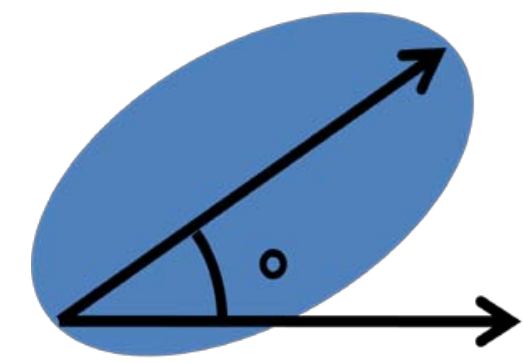

Figure 5-7: Rotation angle of IFOV ellipse.

$$
\begin{gathered}
\text { Equation 10. }- \text { Ellipse rotation angle in radians (ROT_ANGLE). } \\
R O T \_A N G L E=I F\left(L A T-S S C L A T=0,0,\left(\frac{\pi}{2}\right)-\operatorname{ATAN}\left(\frac{N E W L O N-N E W S S C L O N}{L A T-S S C L A T}\right)\right)
\end{gathered}
$$

\subsubsection{Summary of IFOV Ellipse Parameters}

As mentioned earlier, IFOV ellipses were created in ArcGIS using ArcObjects programming. In particular, the IEllipticArc.PutCoordsByAngle method of the ESRI ArcObjects Geometry library was employed to transform the coordinate points of the temperature scans, from the original PPR geometry data files, into IFOV ellipses (refer to 
Appendix B for detailed code). The parameters necessary to create an ellipse using the IEllipticArc.PutCoordsByAngle method are:

- center point

- semi major axis

- minor-major ratio

- rotation angle

In order to obtain these ellipse parameters, specific data was utilized from the original PPR geometry data files, along with new equation calculations and factors such as the PPR IFOV and Io's radius (Table 5). The equations and factors used to calculate the ellipse parameters have been described in the previous 5.1 subsections. 
Table 5. Data, calculations, and factors used to compute ellipse parameters.

\begin{tabular}{|c|c|c|c|}
\hline Ellipse Parameter & Definition & $\begin{array}{l}\text { Original } \\
\text { PPR Data } \\
\text { Values } \\
\text { Utilized }\end{array}$ & $\begin{array}{l}\text { New } \\
\text { Calculations \& } \\
\text { Factors Utilized }\end{array}$ \\
\hline $\begin{array}{l}\text { Center point } \\
\text { (LAT } \\
\text { and NEWLON) }\end{array}$ & $\begin{array}{l}\text { Coordinates of a particular } \\
\text { temperature scan from the } \\
\text { original data files (ellipse center } \\
\text { point) }\end{array}$ & $\begin{array}{l}\text { LAT, } \\
\text { LON }\end{array}$ & NEWLON \\
\hline $\begin{array}{l}\text { Semi major axis } \\
\text { (SMAJ_DD) }\end{array}$ & $\begin{array}{l}\text { Half of the largest axis spanning } \\
\text { between points on opposite } \\
\text { sides of the ellipse }\end{array}$ & $\begin{array}{l}\text { LAT, } \\
\text { LON, } \\
\text { SSCLAT, } \\
\text { SSCLON, } \\
\text { SIPDIS }\end{array}$ & $\begin{array}{l}\text { NEWLON, } \\
\text { NEWSSCLON, } \\
\text { SMIN_KM, } \\
\text { SMAJ_KM, } \\
\text { SMAJ_AD, } \\
\text { PPR IFOV } \\
\text { (2.5 milliradians), } \\
\text { Io’s radius } \\
\text { (1820 km) }\end{array}$ \\
\hline $\begin{array}{l}\text { Minor-major } \\
\text { ratio } \\
\text { (MINMAJ_RAT) }\end{array}$ & $\begin{array}{l}\text { Ratio of the semi minor axis to } \\
\text { the semi major axis }\end{array}$ & $\begin{array}{l}\text { LAT, } \\
\text { LON, } \\
\text { SSCLAT, } \\
\text { SSCLON, } \\
\text { SIPDIS }\end{array}$ & $\begin{array}{l}\text { NEWLON, } \\
\text { NEWSSCLON, } \\
\text { SMIN_KM, } \\
\text { SMIN_AD, } \\
\text { SMIN_DD, } \\
\text { SMAJ_KM, } \\
\text { SMAJ_AD, } \\
\text { SMAJ_DD, } \\
\text { PPR IFOV } \\
\text { (2.5 milliradians), } \\
\text { Io’s radius } \\
\text { (1820 km) }\end{array}$ \\
\hline $\begin{array}{l}\text { Rotation angle } \\
\text { (ROT_ANGLE) }\end{array}$ & $\begin{array}{l}\text { Azimuth angle between: } \\
\text { 1) the line of latitude of the sub- } \\
\text { spacecraft point and } \\
\text { 2) the line connecting the sub- } \\
\text { spacecraft point to the ellipse } \\
\text { center point }\end{array}$ & $\begin{array}{l}\text { LAT, } \\
\text { LON, } \\
\text { SSCLAT, } \\
\text { SSCLON }\end{array}$ & $\begin{array}{l}\text { NEWLON, } \\
\text { NEWSSCLON }\end{array}$ \\
\hline
\end{tabular}




\subsection{Custom ArcGIS Tools}

\subsubsection{Feature Creation}

The planetary science community needs a tool to efficiently create IFOV ellipses and their center point features in ArcGIS. Section 5.1 discusses why it is important to create IFOV ellipses and describes how to calculate the IFOV ellipse parameters using the raw PPR data. Creating ellipse center point features is also important, as center points aid in evaluating the proximity of IFOV ellipses to one another and they are one of the necessary parameters for creating IFOV ellipses. Manual processing of the over 200 Io PPR raw data files into IFOV ellipse and center point feature classes in ArcGIS is timeconsuming and has a great chance for human error. The USGS has also imposed a deadline of 2011 for completion of a GIS containing the full set of processed PPR data for Io. The solution to these issues is a custom tool in ArcGIS that automates the processing of Io PPR data into ellipse and center point features. Building upon the procedures elaborated in Section 5.1, this section describes the construction of this Create Features tool, focusing on its interface and coded functions.

The Create Features dialog box (Figure 5-8) and functionality were created by adding a new Windows form to a new Visual Studio project. Later in this section, the creation of custom tool buttons for ArcMap and ArcCatalog will be discussed, linking the Visual Studio Create Features dialog box to ArcGIS. The following procedures were performed on the new Windows form in Visual Studio:

1. Name dialog box: Under the [Design] tab properties, the default dialog box name was changed from "Form1" to "Create Features".

2. Add TextBoxes: Three TextBoxes were added to the Windows form, in order to store particular file paths. In the properties for the third TextBox, enabling was switched to "False", so that the user would not be able to type in a file path in this TextBox. Instead, the user would be forced to use a button for file browsing (step 4). Since the file path for the third TextBox could specify a multitude of output workspaces for the user (such as a folder, personal geodatabase, file geodatabase, or SDE workspace), a complex set of error messages would need to be generated to account for any possible error in TextBox typing. Therefore, typing was disabled in the third TextBox.

3. Provide labels: Labels were placed above the TextBoxes to provide instructions for the user. The default label text was changed accordingly.

4. Create buttons: A button was placed next to each of the three TextBoxes. These buttons allow the user to browse for files and as such, each button was named "Browse...". A fourth button was added to the bottom of the form and named 
"Create Features". This button was sized larger than the other buttons, as it is the button that creates the ellipse and center point features, once all of the TextBox information has been filled in. (Further details on feature creation are provided later in this section.)

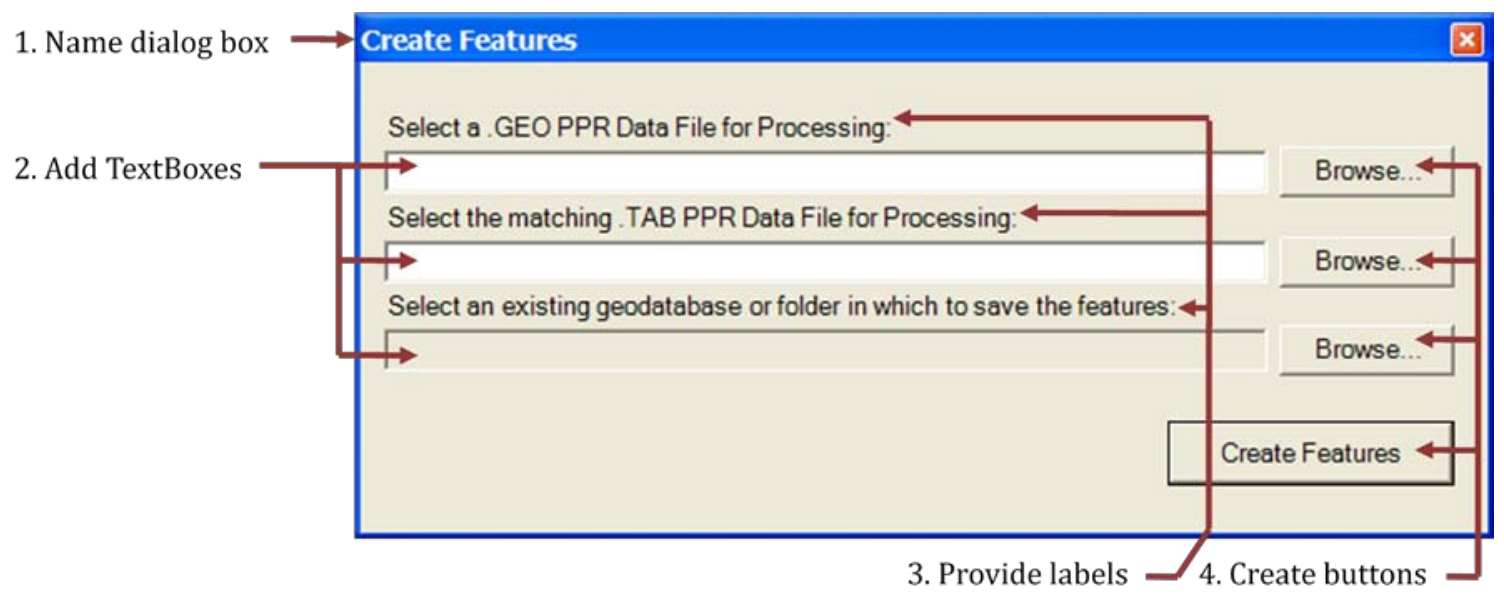

Figure 5-8: Create Features dialog box.

An overview of the coded functions for the Create Features dialog box will be discussed here. For more specifics, please refer to the commented out code in Appendix B. There are two primary sets of functions included in the code, the Browse... button functions and the Create Features button functions.

Upon clicking any of the Browse... buttons in Figure 5-8, a dialog box appears, from which the user can browse and select a file. The dialog box for the first Browse... button only shows files with the extension .geo. The PPR .geo data files contain all the pertinent field values used to calculate the parameters necessary for drawing the IFOV ellipses in ArcMap (Figure 5-1). The dialog box for the second Browse... button only shows files with the extension .tab. The PPR .tab data files contain the average temperature values associated with the IFOV ellipses (Figure 5-9; note that there are no field names listed in the .tab file. The metadata specified the particular column pertaining to the temperature field). The dialog box for the third Browse... button allows the user to select an output workspace for saving the processed feature classes. The output workspace can be a folder, personal geodatabase, file geodatabase, or SDE workspace. The file path, for each file, geodatabase, or folder selected in each Browse... button dialog box, is stored in the respective TextBox that corresponds to that particular Browse... button. The user can also directly type in the file paths of the .geo and .tab files into the first two TextBoxes shown in Figure 5-8. 


\begin{tabular}{|c|c|c|c|c|}
\hline \multicolumn{4}{|c|}{ [ I24PELE_N1.TAB - Notepad } & $\square \times$ \\
\hline \multicolumn{5}{|c|}{ File Edit Format View Help } \\
\hline 0.000 & 13.8 & 130.0 & 0000.0 & $0.00 \mathrm{E} \wedge$ \\
\hline 0.000 & 15.8 & 132.7 & 0000.0 & $0.00 \mathrm{E}$ \\
\hline 0.000 & 15.8 & 132.7 & 0000.0 & $0.00 \mathrm{E}$ \\
\hline 0.000 & 5.9 & 115.1 & 0000.0 & $0.00 \mathrm{E}$ \\
\hline 0.000 & 8.8 & 121.8 & 0000.0 & $0.00 \mathrm{E}$ \\
\hline 0.000 & 11.8 & 127.0 & 0000.0 & $0.00 \mathrm{E}$ \\
\hline 0.000 & 6.7 & 117.3 & 0000.0 & $0.00 \mathrm{E}$ \\
\hline 0.000 & 16.6 & 133.7 & 0000.0 & $0.00 \mathrm{E}$ \\
\hline 0.000 & 10.7 & 125.1 & 0000.0 & $0.00 \mathrm{E}$ \\
\hline 0.000 & 8.7 & 121.5 & 0000.0 & $0.00 \mathrm{E}$ \\
\hline 0.000 & -5.1 & -112.9 & 0000.0 & $0.00 \mathrm{E}$ \\
\hline 0.000 & 11.7 & 126.8 & 0000.0 & $0.00 \mathrm{E}$ \\
\hline 0.000 & 12.6 & 128.3 & 0000.0 & $0.00 \mathrm{E}$ \\
\hline 0.000 & 9.7 & 123.4 & & $0.00 \mathrm{E}$ \\
\hline 0.000 & 3.8 & 108.7 & b000.0 & $0.00 \mathrm{E}$ \\
\hline 0.000 & 6.7 & 117.3 & 0000.0 & $0.00 \mathrm{E}$ \\
\hline 0.000 & 171.1 & 208.3 & 0000.0 & $0.00 \mathrm{E}$ \\
\hline 0.000 & 1.8 & 99.3 & 0000.0 & $0.00 \mathrm{E}$ \\
\hline 0.000 & 4.7 & 111.8 & 0000.0 & $0.00 \mathrm{E}$ \\
\hline 0.000 & 4.7 & 111.8 & 0000.0 & $0.00 \mathrm{E}_{\checkmark}$ \\
\hline$<$ & & & & $>$ \\
\hline
\end{tabular}

Figure 5-9: Average temperature values in PPR raw data records (.tab files) associated with IFOV ellipses.

Upon clicking the Create Features button in Figure 5-8, many objects are created, leading up to and including the creation of the ellipse and center point features:

1. Streamreaders: Two Streamreader objects are created for reading lines of information from text files; one to read .geo files and one to read .tab files. The header info (i.e., the first three lines) is skipped in the reading of the .geo files. The Streamreaders iterate through the remaining lines of the .geo and tab files, parsing the fields based on white space and special characters, such as “/” and “+”. The fields are then stored in two temporary arrays. As mentioned in Section 4.4, there are some records that need to be removed; they contain invalid data values marked "666" or null/negative temperature values. In the code, these records are programmatically skipped over and therefore, are not stored in the temporary arrays. Finally, the two Streamreaders are closed.

2. Data table: An empty data table in memory is also created by the code, with columns named as follows:

- ADJ_SCLK (this is the unique identifier for each record, found in both the .geo and .tab files - see Section 4.3)

- LAT

- LON 
- NEWLON

- SIPDIS

- SSCLON

- NEWSSCLON

- SSCLAT

- SMIN_KM

- SMIN_AD

- SMIN_DD

- SMAJ_KM

- SMAJ_AD

- SMAJ_DD

- MINMAJ_RAT

- ROT_ANGLE

- TEMP

Each data table column is filled with the corresponding field values from the temporary arrays or new calculated values (as described in Section 5.1).

3. Ellipse center point features \& attribute table: The ellipse center point features are created by declaring: a) the geometry type as "point", b) the data table NEWLON field values as the X point coordinates and the data table LAT field values as the $\mathrm{Y}$ point coordinates, and c) the spatial reference as the Io_2000 geographic coordinate system. An attribute table for the point features is also created in the code, with OBJECTID and Shape field information filled in. Additional fields are named the same as the columns in the data table and are then filled with the corresponding data table field values. These fields consist of the TEMP field and the ellipse parameter fields. The ellipse center point feature classes are saved with the same name as the original geo raw data files, except “_pt” is added to the name. (The feature class names could have also been referenced to the original .tab raw data files, as the .geo and .tab files were saved with the same name at the time of download, as mentioned in Section 4.3.) Additionally, the files are named with the appropriate extension matching the output workspace location: .shp, .mdb, .gdb, or .sde.

4. Ellipse features \& attribute table: The ellipse features are created by means of the IEllipticArc.PutCoordsByAngle programming method as described in Section 5.1, which uses the LAT, NEWLON, SMAJ_DD, MINMAJ_RAT, and ROT_ANGLE field values of the point feature attribute table. The geometry type is specified as "polygon" and the spatial reference as the Io_2000 geographic coordinate system. An attribute table for the polygon features (i.e., the ellipse features) is also created in the code, with OBJECTID, Shape, 
Shape_Length, and Shape_Area field information filled in. All field names and values from the point feature attribute table are then added to the polygon feature attribute table. The ellipse feature classes are saved with the same name as the original .geo raw data files, except “_poly” is added to the name. Additionally, the files are named with the appropriate extension matching the output workspace location: .shp, .mdb, .gdb, or .sde.

5. Error and confirmation messages: Various error messages are also coded to appear if an error occurred. Figure 6-5 in Chapter 6 represents a sampling of such message boxes. A message box confirming the completion of processing was also coded to appear following the creation of the new ellipse and center point features (Figure 6-6).

Of particular note, the GC.Collect programming method is used to collect garbage after executing each of the following sets of code: 1) processing of the data table in memory, 2) processing of the point features (i.e., ellipse center points), and 3) processing of the polygon features (i.e., ellipses). Without the GC.Collect method, the processing of personal geodatabase point and polygon features times out at 169 features and a variety of error messages appear. A search on the ESRI Support Center webpage (http://support.esri.com/) for one of the error messages that read "Cannot open any more tables” led to discovery of the GC.Collect method.

A new ArcMap tool button and new ArcCatalog tool button are also created in the code (see Appendix C for detailed instructions and Appendix B for the resulting code), and linked with the Create Features dialog box and associated code. Clicking the new custom tool button in either ArcMap or ArcCatalog triggers the loading of the Create Features dialog box. Following the instructions in Appendix C, the Desktop ArcMap Command was chosen for the new ArcMap button, while the Desktop ArcCatalog Command was chosen for the new ArcCatalog button. In the resulting stubbed out code, the default values were changed for the tools' category, caption, message, tooltip, and name properties. The default .bmp tool button images were also changed to the buffer.bmp image (Figure 5-10) in order to represent ellipse and center point features. The purple background color of the .bmp image is automatically set to appear clear on the actual tool button.

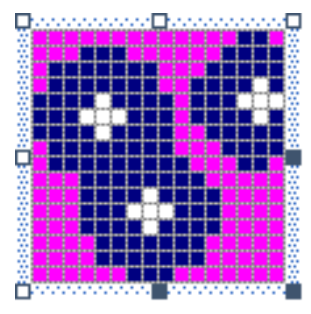

Figure 5-10: Buffer.bmp tool button image. 


\subsubsection{Temperature-specific Symbology}

In addition to automatically creating the IFOV ellipses and their center point features in ArcGIS, the client requested a tool that would aid planetary scientists in effectively distinguishing the corresponding temperature data in ArcMap. Aptly, once the IFOV ellipses are created, the visualization capabilities of ArcMap could be utilized to convey the approximate temperature on Io's surface at each of the IFOV ellipses. Repeated manual symbolization of the over 100 IFOV ellipse layers that will be created for Io, based on temperature, is time-consuming and has a great chance for human error. The solution to these issues is a custom tool in ArcGIS that automates the processing of new temperature-specific symbology for IFOV ellipse layers. This section describes the creation of this Set Temperature Symbology tool, focusing on its interface and coded functions.

The Set Temperature Symbology dialog box (Figure 5-11) and functionality were created by adding another Windows form to the existing Visual Studio project. Later in this section, the creation of a custom tool button for ArcMap will be discussed, linking the Visual Studio Set Temperature Symbology dialog box to ArcGIS. The following procedures were performed on the new Windows form in Visual Studio:

1. Name dialog box: Under the [Design] tab properties, the default dialog box name was changed from "Form2" to "Set Temperature Symbology".

2. Add ComboBox: A ComboBox was added to the Windows form, giving users the ability to select a layer in which to set the symbology.

3. Provide label: A label was placed above the ComboBox to provide instruction for the user. As such, the default label text was changed from "Label1" to "Set temperature symbology for the following layer:”.

4. Create button: A button was added to the bottom of the form and named "Set New Symbology". This button classifies the temperature data into ranges and assigns the appropriate symbology for each range. (Further details on feature classification are provided later in this section.) 


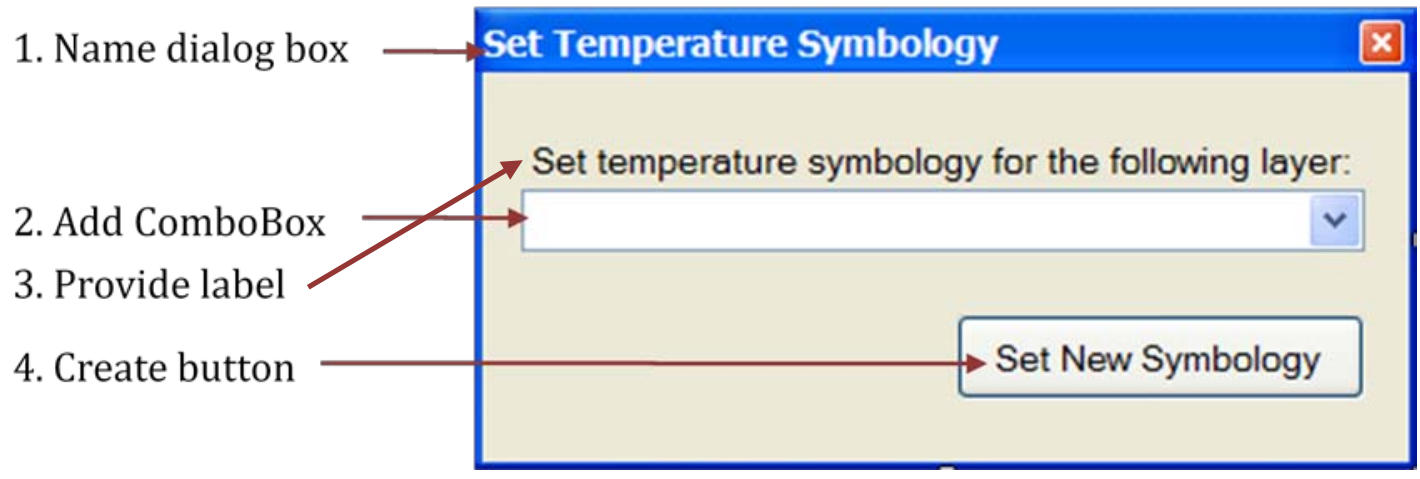

Figure 5-11: Set Temperature Symbology dialog box.

An overview of the coded functions for the Set Temperature Symbology dialog box will be discussed here. For more specifics, please refer to the commented out code in Appendix B. There are two primary sets of functions included in the code, the ComboBox functions and the Set New Symbology button functions.

Upon loading of the Set Temperature Symbology dialog box, the valid selectable layers for symbolizing are compiled and listed in the drop-down ComboBox in Figure 511. The selectable layers are polygon feature layers in the active map that contain a valid "TEMP" field for classification.

Upon clicking the Set New Symbology button in Figure 5-11, the selected layer in the previous ComboBox is processed. A ClassBreaksRenderer object is used in the code to:

1. Divide the layer's range of temperature values into classes: In consultation with the client, class ranges for the "TEMP” field values were specified as follows:

- $<100 \mathrm{~K}$

- $100-110 \mathrm{~K}$

- $110-120 \mathrm{~K}$

- $120-130 \mathrm{~K}$

- $130-140 \mathrm{~K}$

- $140-150 \mathrm{~K}$

- $150-160 \mathrm{~K}$

- $160-170 \mathrm{~K}$

- $170-180 \mathrm{~K}$

- $>180 \mathrm{~K}$

Class labels were set in the code to display these specific ranges in ArcMap's table of contents. Accordingly, the number of classes was set to 10 and class breaks were defined as $100 \mathrm{~K}, 110 \mathrm{~K}, 120 \mathrm{~K}, 130 \mathrm{~K}, 140 \mathrm{~K}, 150 \mathrm{~K}, 160 \mathrm{~K}$, 
$170 \mathrm{~K}, 180 \mathrm{~K}$ and $600 \mathrm{~K}$ in the code. (The value of $600 \mathrm{~K}$ is arbitrary; it was deemed there would be no temperature values greater than $600 \mathrm{~K}$ in the PPR datasets.)

2. Assign a different symbol to each class, by setting the following:

a) Symbol colors: The symbol color for each temperature class was defined in the code, using Red-Green-Blue (RGB) color values (Figure 5-12). These colors were taken from a 10-class diverging spectral color scheme chosen from ColorBrewer (Brewer, 2002), an online tool that offers color schemes for thematic mapping. A diverging color scheme was used to emphasize the high and low ends of the temperature range with dark colors, and the midtemperature range with light, bright colors. In conjunction, the spectral color scheme was chosen as it is widely-known for being representative of a hot-tocold range. Furthermore, the variety of different colors in the spectral color scheme helps to greater distinguish temperature classes for visualization.

b) Symbol outline properties: Next in the code, the polygon symbol outlines for each class were designated to receive the aforementioned symbol colors, with each polygon symbol's outline width set to 1 . (The value of 1 is arbitrary; this value, however, seemed ideal for visualization in ArcMap, as the width was not too thick and heavy, and also not so thin as to be undetectable against a color background image of Io's surface).

c) Symbol fill properties: Each polygon symbol's fill was then set to hollow. Since there are many overlapping polygon features, filling these polygon symbols completely with color would result in most of the previously drawn features being completely obscured by the features drawn later in ArcMap. By having a hollow fill, most of the elliptical shape of the polygons can still be seen (Figure 5-13).

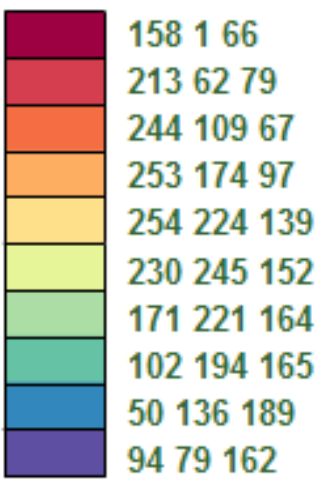

Figure 5-12: Temperature color ramp with associated RGB values. 


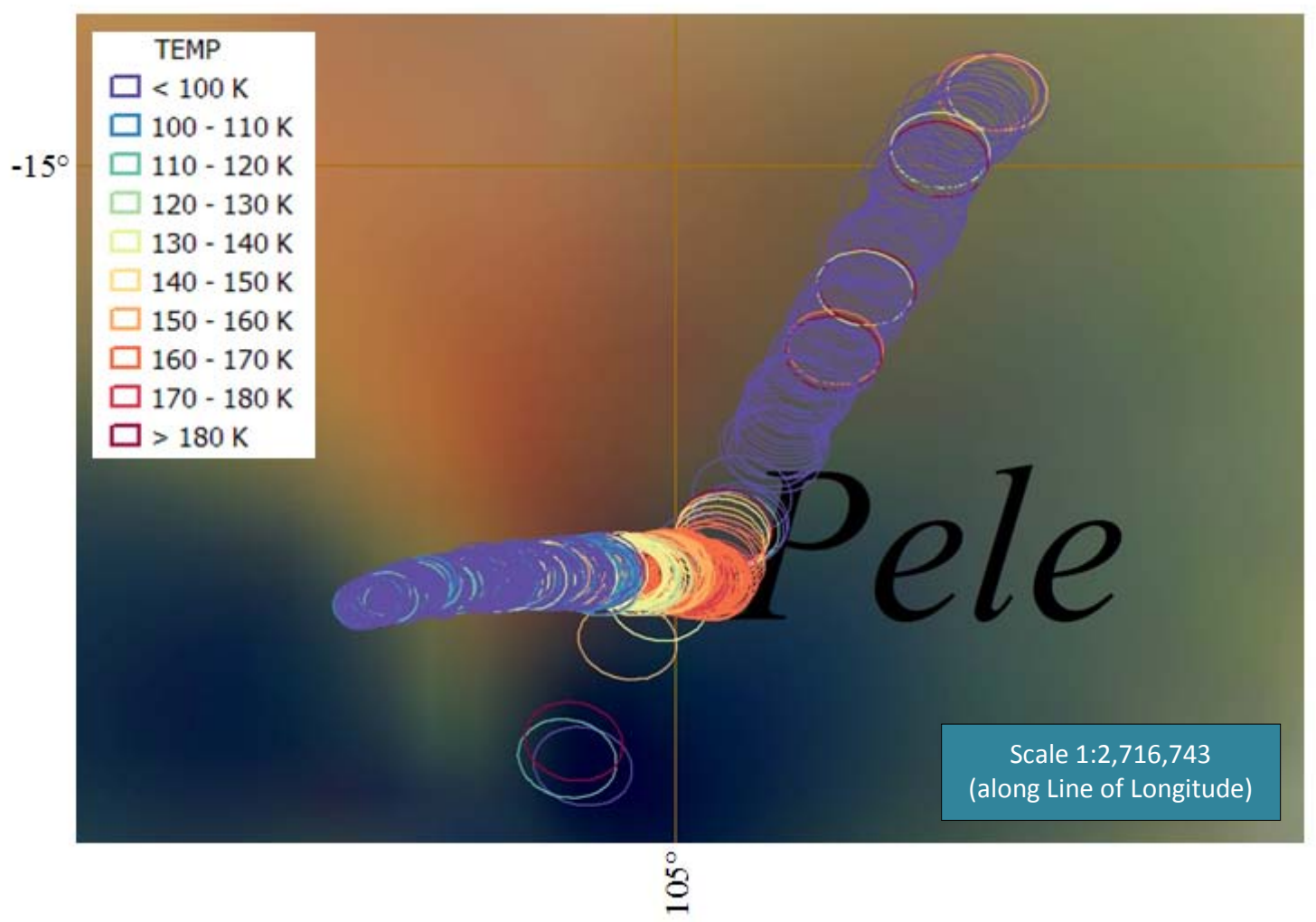

Figure 5-13: New temperature-specific symbology of a selected layer.

The map's active view area and the table of contents are then set to refresh in the code, resulting in the display of the new class ranges and associated symbology in ArcMap. A message box confirming the completion of processing is also coded to appear (Chapter 6, Figure 6-7). A close-up of the newly symbolized temperature scans (Figure 5-13) reveals a cluster of high temperatures at Pele at approximately $-18^{\circ}, 105.5^{\circ}$, indicative of eruptive activity. However, it is to be noted that non-clustered single or paired high temperature scans were most likely caused by noise impacting the detector and are not significant (see Section 4.3 for details).

A new ArcMap tool button is also created in the code (see Appendix $C$ for detailed instructions and Appendix B for the resulting code), and linked with the Set Temperature Symbology dialog box and associated code. Clicking the new custom tool button in ArcMap triggers the loading of the Set Temperature Symbology dialog box. Following the instructions in Appendix C, the Desktop ArcMap Command was chosen for the new ArcMap button. In the resulting stubbed out code, the default values were changed for the tool's category, caption, message, tooltip, and name properties. The default .bmp tool button image was also changed to the buffer.bmp tool image, with colors altered (Figure 5-14), in order to represent ellipses that were color-coded by temperature. The 
purple background color of the .bmp image is automatically set to appear clear on the actual tool button.

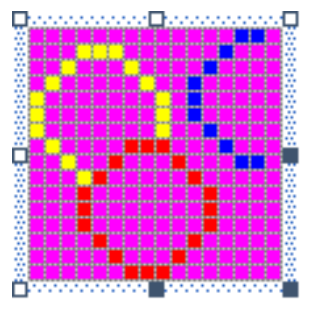

Figure 5-14: Altered Buffer.bmp tool button image.

For organization purposes in ArcMap, a toolbar is also created in the code to house the Set Temperature Symbology ArcMap tool button and the Create Features ArcMap tool button (see Appendix $\mathrm{C}$ for detailed instructions and Appendix B for the resulting code). Following the instructions in Appendix C, the Base Toolbar was chosen for the new ArcMap toolbar. In the resulting stubbed out code, the ArcMap Set Temperature Symbology and Create Features tool buttons were added and set to appear on the toolbar interface.

\subsubsection{Custom Install Program}

Lastly, a custom install program for the ArcGIS Io PPR Data Processing toolkit (containing the toolbar, Create Features tool, and Set Temperature Symbology tool) was created as an executable file (see Appendix D for detailed instructions). The user is able to install the custom Io PPR Data Processing toolkit directly from this executable file, eliminating the need for having Microsoft Visual Studio or Visual Basic installed on the user's computer and running the toolkit code from there. The only prerequisite is that the user must have ArcGIS 9.3 installed on their computer. The necessary Visual Basic .NET components of the toolkit are installed on the user's computer when the executable file is launched.

\subsection{Summary}

All the phases of the project implementation were discussed in this chapter. The data were transformed from their original coordinates to elliptical shapes that more accurately represent the area scanned on the ground surface of Io by the PPR instrument at that particular moment in time. The parameters necessary for creating these scanned elliptic areas (IFOV ellipses) were obtained through a series of data transformation calculations. The specific necessary parameters are the ellipse center point, semi major axis, minormajor ratio, and rotation angle. For data visualization purposes, it was also necessary to classify the ellipse layers by average temperature at each of the IFOV ellipses. Two custom tools were created in ArcGIS to: 1) create new ellipse and center point feature 
classes from the raw data, utilizing the aforementioned parameters, and 2) generate new temperature-specific symbology for the ellipse layers. Automating processes with custom tools saves time over manually processing a great number of files/layers and reduces the chance for human error. For each custom tool, a user interface was first created and then functions were coded for, to be performed with certain interface load or click events. Finally, a custom install program was created to wrap up the toolkit containing the toolbar, the Create Features tool, and the Set Temperature Symbology tool. 


\section{Chapter 6 - Results and Analysis}

The goals of the project were achieved through creation of new custom tools in ArcGIS. Instantaneous field of view (IFOV) ellipses and center point features are generated in ArcGIS through the automated processing functions of the Create Features tool, while new temperature-specific symbology is generated for the IFOV ellipses in ArcGIS through the automated processing functions of the Set Temperature Symbology tool. The tools provide a simple design and clear, concise, interfaces to users who are generally familiar with the two types of PPR data files to be processed, .geo and .tab files, and the two functions that need to be performed, creating feature classes and setting new symbology for the ellipse layers. There are over 200 ellipse and ellipse center point feature classes to be created for the areas scanned on Io by the PPR instrument, with new symbolizations for all ellipse layers. As mentioned in the previous chapter, automation saves time over manually processing a great number of files/layers and reduces the chance for human error. By using the Create Features and Set Temperature Symbology tools, the project client, Dr. Rathbun, and her team will definitely be able to process the full set of PPR data for Io before the USGS deadline of 2011. This chapter discusses installation of the toolkit, what the final tools look like in ArcGIS, how they function to the user, the tool processing times, and issues encountered in creating the tools.

\subsection{Toolkit Installation}

The final Io PPR Data Processing toolkit is wrapped in an executable file. When the user runs the executable toolkit file, the Io PPR Data Processing toolbar, the Create Features tool, and the Set Temperature Symbology tool are installed to the hard drive at a location specified by the user.

The toolbar can be accessed in ArcMap under Tools $\rightarrow$ Customize. Under the Toolbars tab in the Customize dialog box (Figure 6-1), scroll under "Toolbars:" and click the box next to "Io PPR Data Processing”, and then click the Close button. This procedure adds the toolbar to ArcMap. 


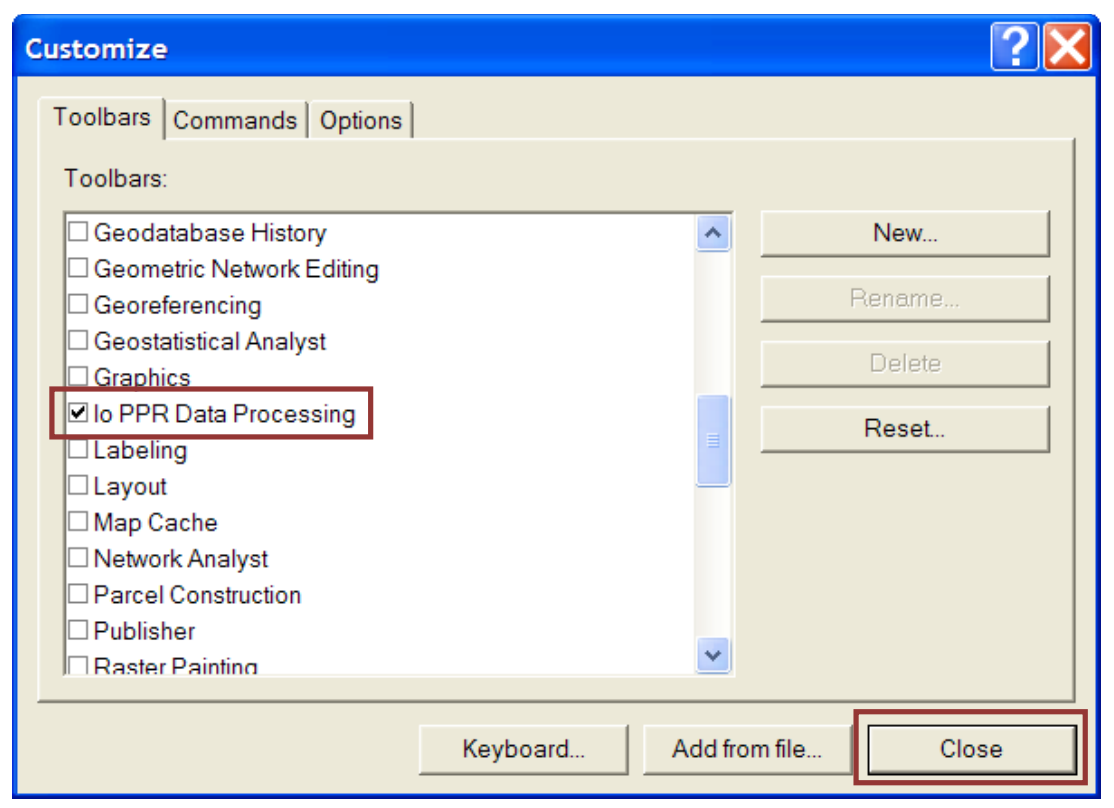

Figure 6-1: Dialog box screenshot showing how to add the Io PPR Data Processing toolbar to ArcMap.

The tools can be accessed in ArcMap under Tools $\rightarrow$ Customize. Under the Commands tab in the Customize dialog box (Figure 6-2), scroll under "Categories:” and click “Io PPR Data Processing”. Then drag each tool from the "Commands:” list to the newly added toolbar and click the Close button.

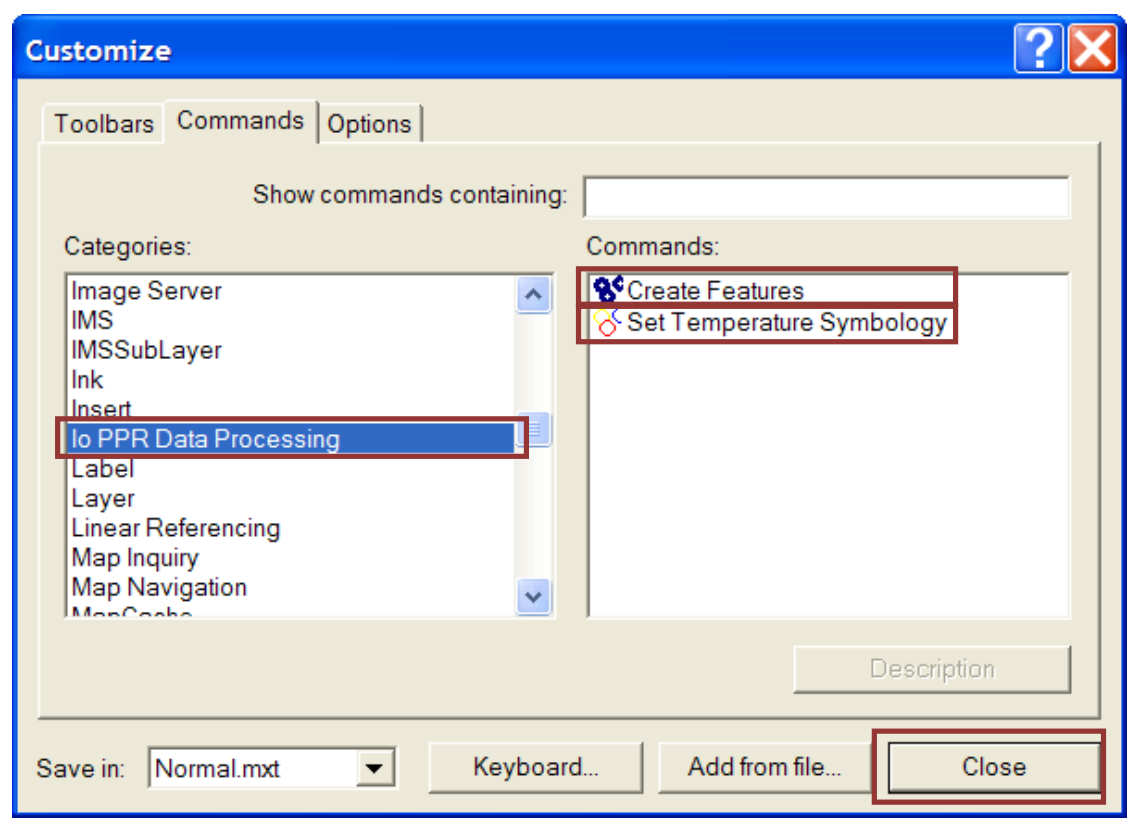

Figure 6-2: Dialog box screenshot showing how to add the Create Features and Set Temperature Symbology tools to an ArcMap toolbar. 
Similarly, the Create Features tool can also be accessed in ArcCatalog under Tools $\rightarrow$ Customize. Under the Commands tab in the Customize dialog box (Figure 6-3), scroll under "Categories:" and click "Io PPR Data Processing”. Then drag the Create Features tool from the "Commands:" list to any existing toolbar and click the Close button. The Set Temperature Symbology tool is not available in ArcCatalog as it only works in ArcMap; symbology can only be created and saved in an ArcMap layer and not within the feature class files.

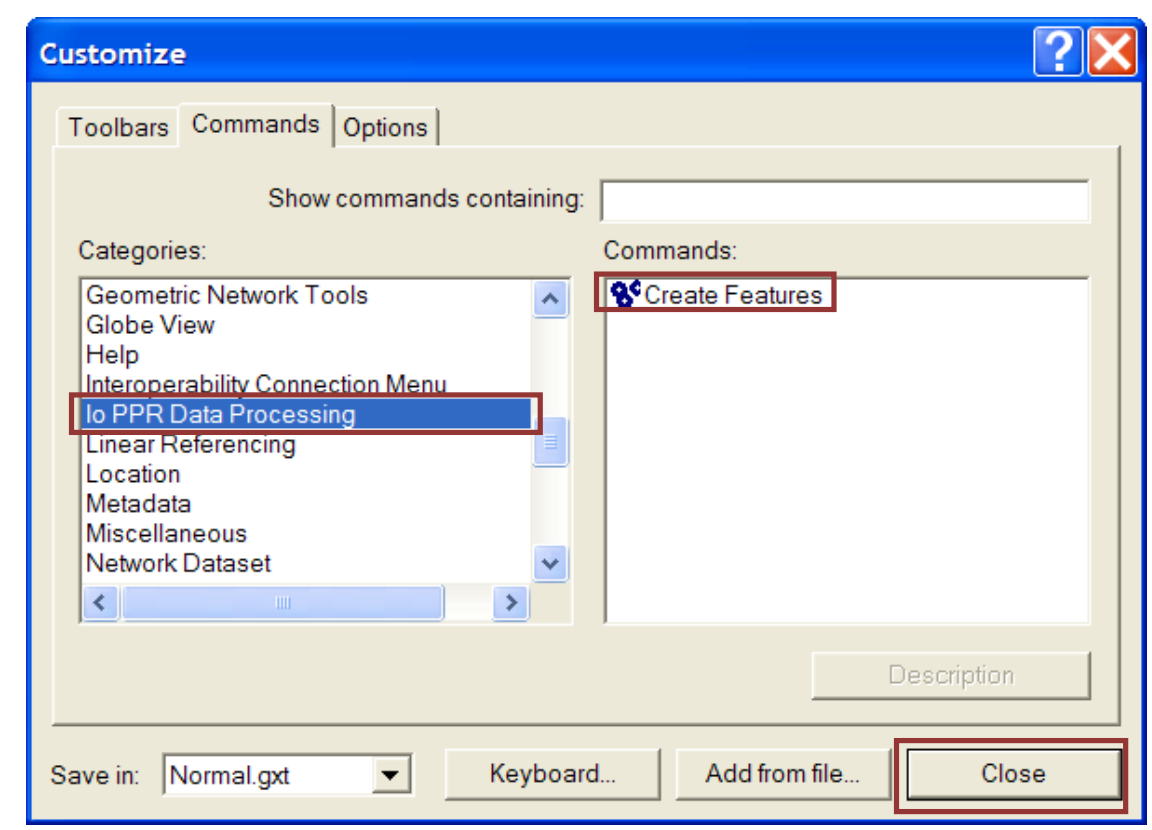

Figure 6-3: Dialog box screenshot showing how to add the Create Features tool to an ArcCatalog toolbar.

The text associated with the button images can be turned on by first clicking Tools $\rightarrow$ Customize and then right-clicking the button image and selecting "Image and Text”. The resulting toolbar in ArcMap is shown in Figure 6-4.

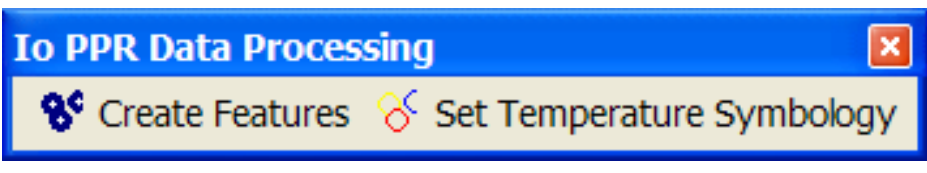

Figure 6-4: Io PPR Data Processing toolbar.

\subsection{Tool Usage}

Once the toolkit has been installed by the user, processing raw PPR data files with the tools is a very simple procedure. When the user clicks on the Create Features tool button, the Create Features dialog box (Figure 5-8) appears. The user then browses or types in 
file paths to the .geo and .tab data files for processing. Next, the user selects an existing geodatabase or folder in which to save the features and clicks the Create Features dialog box button. If there is an error, an error message box appears. An error message is a helpful way to inform the user of why the procedure cannot correctly proceed. The user must correct the error(s) and click the Create Features dialog box button again for data processing to start over. The possible types of error messages are shown in Figure 6-5. The user may have specified a file path that does not exist or that doesn't specify a particular file. The user might otherwise have specified a file with the wrong extension or not have filled in all information before clicking the Create Features button. The user must also be careful to select the correct matching .geo and .tab file pair; the matching files have the same filename, just different extensions (i.e., geo or .tab). When using ArcMap and there are no errors (or errors have been corrected), ArcMap updates the table of contents with the ellipse (polygon) and center point features and displays them on the map. Simultaneously, a message box saying, "Files successfully written to geodatabase", appears, informing the user of a successful data translation (Figure 6-6). Using the Create Features tool in ArcCatalog, a similar sequence of events occurs; ArcCatalog is updated with the new point and polygon features and the same message box appears saying, "Files successfully written to geodatabase".

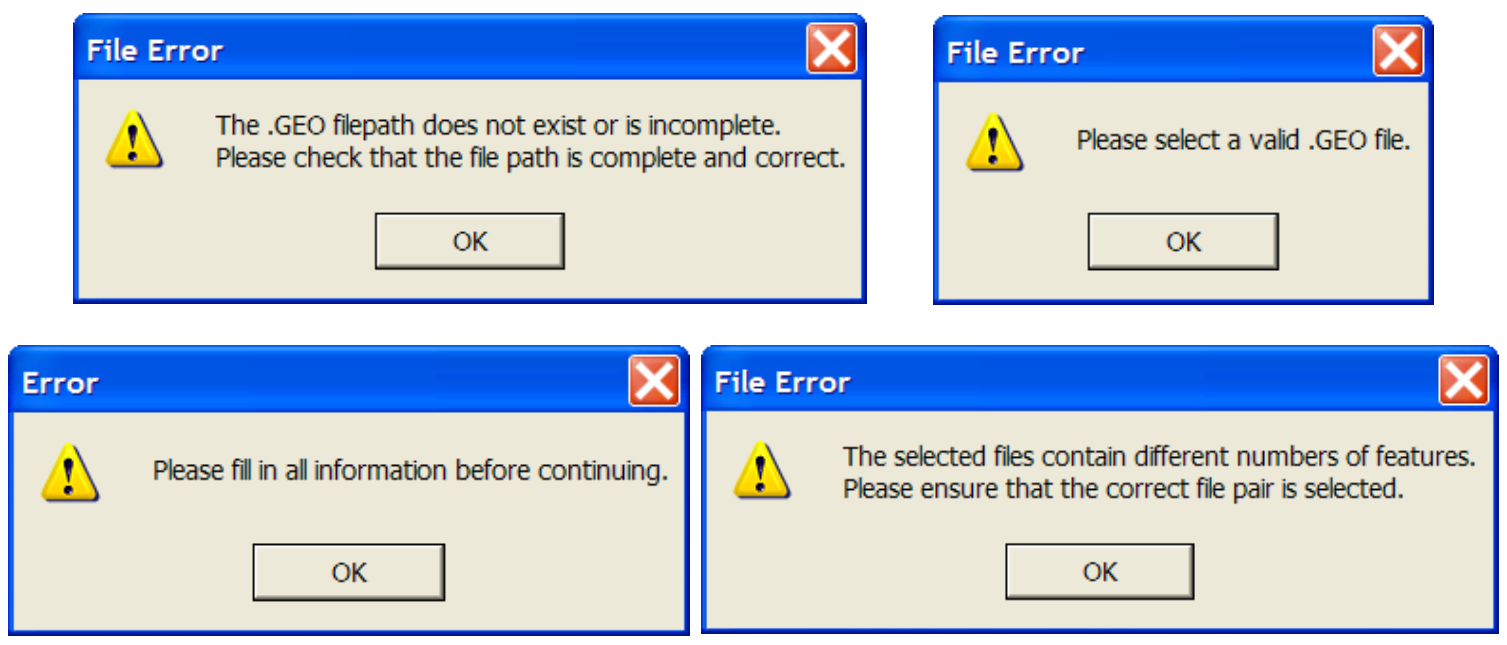

Figure 6-5: Possible types of error messages from the Create Features tool. 


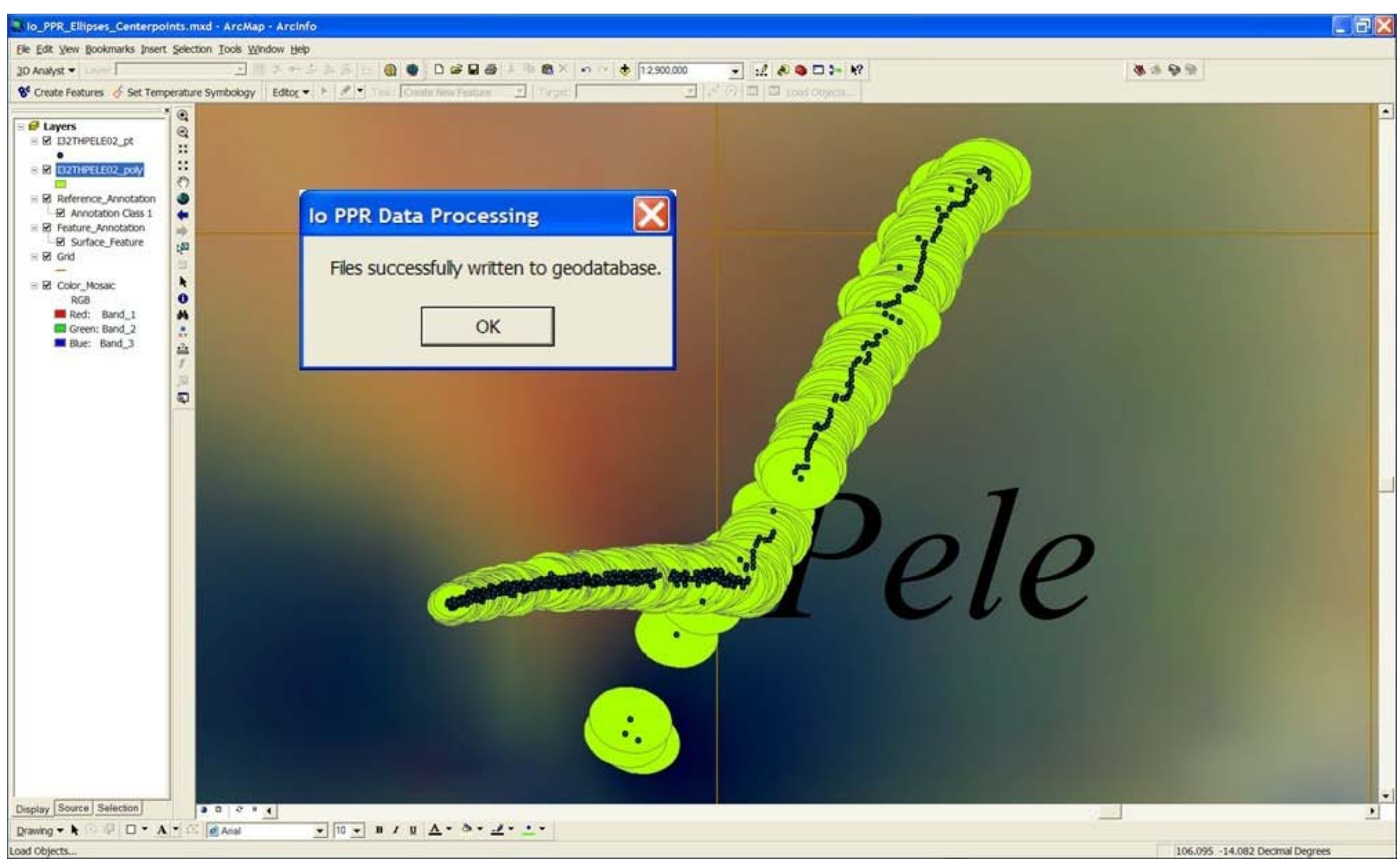

Figure 6-6: Ellipse and center point features in ArcMap. 
When the user clicks on the Set Temperature Symbology button, the Set Temperature Symbology dialog box (Figure 5-11) appears. From the ComboBox dropdown list, the user then selects the layer in which to set the temperature symbology. Next, the user clicks the Set New Symbology dialog box button. The ArcMap display and table of contents update with the new symbology and a message box appears saying, “The map has been updated with the new symbology” (Figure 6-7). 


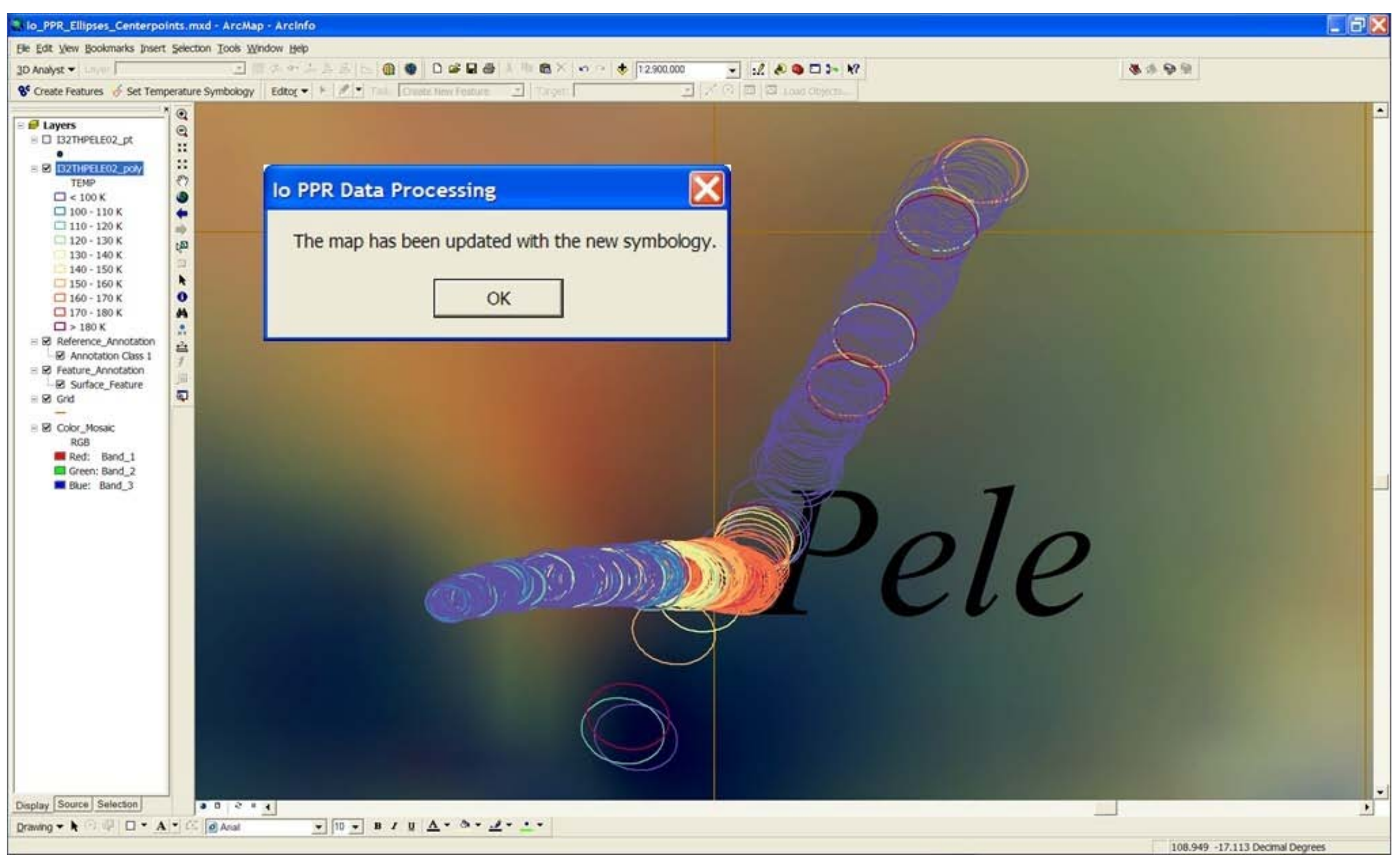

Figure 6-7: New temperature-specific symbology in ArcMap. 


\subsection{Tool Performance}

Using the Create Features tool, eleven IFOV ellipse feature classes and eleven corresponding ellipse center point feature classes, representing the temperature scans for the Pele-Pillan region of Io, were created and saved in a file geodatabase. An ArcGIS file geodatabase was chosen for the storage of the feature classes as it has virtually no size limitation, as described in Section 4.2. However, the client is awaiting a decision on the final file format from the principal investigation team at Arizona State University. The flexibility of the Create Features tool in saving features as shapefiles or different types of geodatabase files is useful in this situation.

For the Create Features tool, the average processing time to create a file geodatabase feature class of ellipses and corresponding center point features was 1 minute and 3 seconds. Processing of the smallest file pair (the I27PELE_01 .geo and .tab files), with just 21 data records, took 34 seconds; while processing of the largest file pair (the I31PELEDK01 .geo and .tab files), with 7434 data records, took 4 minutes and 11 seconds. Processing times for creating personal geodatabase files were similar, while processing times for creating shapefiles varied and took up to six times longer.

In comparison, manually processing the smallest .geo and .tab file pair, with the help of the IEllipticArc.PutCoordsByAngle programming method in ArcMap's Visual Basic Editor, took approximately 30 minutes to complete. The processes that were used included:

- saving the pertinent data from a .geo and .tab file pair in an Excel file with fixed width formatting

- adding new columns of equations in Excel for the ellipse parameters

- adding the Excel file to an ArcMap document, creating an XY Events layer set to the Io_2000 geographic coordinate system, and exporting the data as a point shapefile

- creating a polygon (ellipse) shapefile from the point shapefile, using the IEllipticArc.PutCoordsByAngle programming method in ArcMap’s Visual Basic Editor

- joining the attribute data from the point shapefile to the polygon shapefile

To the author's best knowledge, there are no manual steps that would substitute for the IEllipticArc.PutCoordsByAngle programming method; otherwise a complete manual method would have been tested. Exporting the point and polygon shapefiles as feature classes to a file or personal geodatabase added about 1 minute and 30 seconds to the total processing time. A .geo and .tab file pair with a greater number of data records to be processed will require a longer processing time than the aforementioned 30 minutes. The 
processing time can also vary greatly due to: 1) the familiarity level and speed of the person processing the data (the author is very familiar with the data, the ellipse parameter calculations, and operations in Excel and ArcGIS; therefore, the author was able to conduct the manual steps, mentioned above, fairly quickly), 2) time spent finding and deleting records containing invalid data values marked “666” or null/negative temperature values, and 3) time spent checking for any human errors introduced into the processed files.

Manual symbolization of an ellipse layer took approximately 9 minutes and 30 seconds compared to a few seconds with the Set Temperature Symbology tool. The manual process involved setting the 10 class ranges, the corresponding labels, and specifying the temperature-specific symbol outline color (with hollow fill) for each class range. The process can become laborious when symbolizing over 100 ellipse layers and time must also be spent to check for any errors introduced into the symbology.

Using the Create Features and Set Temperature Symbology tools saves much time over manually performing the same tasks achieved by these automated tools. Automated tasks clean up the data quickly and minimize human error, resulting in even more time saved. The saved time adds up very quickly when running the tools over 100 times to process all of Io's PPR data. Persons using the tools also only need to have a very basic level of familiarity with the data and tool functions, whereas the manual process would require a more in-depth understanding and an instructional script.

\subsection{Tool Development}

Several tool functions were difficult to code, as the author was fairly new to Visual Basic and ArcObjects programming. These functions included linking the Visual Basic code with ArcGIS, skipping the reading in of header information and invalid data values from the .geo and .tab files, transferring the data read by the Streamreader to new feature classes, saving the feature classes as different file types, and filling the ComboBox in the Set Temperature Symbology dialog box with valid selectable layers that contain temperature values. In order to figure out how to perform these functions, I consulted the ESRI ArcGIS Desktop Resource Center (http://resources.esri.com/arcgisdesktop/), the Microsoft Developer Network Library (http://msdn.microsoft.com/enus/library/default.aspx), and the ESRI Press (2003) Getting to Know ArcObjects book; all of which contained many helpful programming component definitions and sample code. Nate Strout, an experienced ArcGIS programmer at the Redlands Institute, provided expert advice and further assistance. 


\subsection{Summary}

This chapter described the typical user experience in employing the Io PPR Data Processing toolkit, as well as average data processing times and issues encountered in creating the tools. After installing the toolkit from an executable file, the user initializes the toolbar and tools in ArcGIS through the Tools $\rightarrow$ Customize menu in ArcMap and ArcCatalog. Next, the user clicks on the Create Features tool button, and a dialog box appears. The user fills in all of the text boxes and clicks on the Create Features button in this dialog box. New IFOV ellipses and center point features are then created. Similarly, in order to create new symbology for the IFOV ellipse layers, the user clicks on the Set Temperature Symbology tool button, and a dialog box appears. The user selects a layer for symbolizing and clicks on the Set New Symbology button in this dialog box. New temperature-specific symbology is then created for the selected ellipse layer. The average processing time to create a file geodatabase feature class of ellipses and corresponding center point features, using the Create Features tool, was 5 minutes. Symbolization of an ellipse layer using the Set Temperature Symbology tool took only a few seconds. The automated tools minimized any potential error and saved much time over manually processing the PPR data. During the implementation, ESRI Resource Center and Microsoft Developer Network Library websites were found to be very useful programming resources. In addition, input from an expert programmer helped in coding several complex functions. 


\section{Chapter 7 - Conclusions and Future Work}

Jupiter's moon Io is of great interest, as it is the most volcanically active body in the solar system. A GIS of Io's PPR surface temperature data will help scientists in visualizing and geospatially analyzing Io's volcanic activity. As such, the goal of this project was to develop a toolkit to efficiently process surface temperature data of Jupiter's moon Io for visualization in a GIS. There were no out-of-the-box ArcGIS tools available to process this data that was captured by the Galileo spacecraft's Photopolarimeter-Radiometer (PPR) instrument. The previous methods for processing PPR data are quite lengthy and ineffective for processing a large volume of datasets. Therefore, creation of a new custom toolkit was deemed appropriate, in order to meet the July 2011 USGS deadline for completion of a GIS containing the entire set of PPR data for Io.

A custom Create Features tool was created to transform the data from their original coordinates to elliptical shapes that more accurately represent the area scanned on the ground surface of Io by the PPR instrument at that particular moment in time. Specific ellipse parameters were obtained through a series of data transformation calculations. These parameters include the ellipse center point, semi major axis, minor-major ratio, and rotation angle. The IEllipticArc.PutCoordsByAngle programming method of the ESRI ArcObjects Geometry library uses these parameters to create the scanned elliptic areas (IFOV ellipses) in ArcGIS. A user interface for the Create Features tool was created in Visual Studio and functions were coded for, to be performed with certain interface load and click events. These functions used Visual Basic and ArcObjects programming, including the mentioned IEllipticArc.PutCoordsByAngle method, to create: 1) ellipses and center point features, 2) corresponding attribute tables containing the important temperature data, and 3) error notification/confirmation of completed processing messages.

A custom Set Temperature Symbology tool was also created to classify the ellipse layers by average temperature at each of the IFOV ellipses. A user interface for the Set Temperature Symbology tool was developed in Visual Studio and functions were programmed, to be performed with certain interface load or click events. These functions used Visual Basic and ArcObjects programming to create: 1) new color-coded temperature-specific symbology for the ArcMap ellipse layers, 2) corresponding table of contents labels for the temperature classes, and 3) a message box confirming the completion of processing.

Visual Basic and ArcObjects programming were also used to create a toolkit to house the Create Features and Set Temperature Symbology tools and to wrap up the entire toolkit as an executable file. 
Some helpful programming component definitions and sample code were found by consulting the ESRI Resource Center and Microsoft Developer Network Library websites, the ESRI Press (2003) Getting to Know ArcObjects book, and an experienced programmer.

The completed custom Io PPR Data Processing toolkit for ArcGIS met all of the client's functional requirements. The toolkit can be installed on multiple machines for use within the client's desktop computing environment. The Create Features tool satisfies the following essential functions:

- enables user to select a PPR text file pair to process

- generates instantaneous field of view (IFOV) ellipse features, representing temperature scans of Io's surface by the Photopolarimeter-Radiometer instrument

- enables user to select a preexisting output workspace in which to save the features

The Set Temperature Symbology tool satisfies these important functions:

- enables user to select an ellipse layer to symbolize

- generates symbolized ellipse layers, color-coded and labeled by temperature range

The Io PPR Data Processing toolkit also met all of the non-functional technical, operational, and transitional requirements imposed on the software system. In response to the technical requirements, the toolkit can be installed from an executable file and was designed with a simple-to-use interface for ArcInfo 9.3. The toolkit satisfies a gamut of operational requirements. If an incorrect file path is specified by a user in an interface text box, an error message appears. When all information is entered correctly, the automated toolkit creates the new features and symbology quickly, automatically setting Io_2000 as the geographic coordinate system for the features and performing certain data clean-up functions. When processing is complete, a confirmation message appears. In terms of transitional requirements, a meeting was arranged with the client to demonstrate toolkit usage and to give the client the completed toolkit and a copy of this detailed report, which will serve as documentation for the toolkit. For data organization and storage, a geodatabase was created and delivered to the client, containing the processed PPR ellipses and center point feature classes for Io's Pele-Pillan region and the ancillary reference files. Lastly, the client was provided with an ArcMap document (.mxd file) that is linked to these feature classes and contains the new temperature-specific symbology of the ellipse features. 
However, as with any project, there are always improvements that can be made. Due to the one-year time constraint on this project, the scope was limited to expressly meet the immediate processing needs, or functional requirements, of the client and other potential users. Potential useful extensions to this work would include options on the tool interface for batch processing and naming of output files, as well as a separate tool for spatial averaging of the temperature data between overlapping and proximate ellipses.

The interfaces for the Create Features and Set Temperature Symbology tools could be upgraded to provide more functionality. Enabling batch processing for the Create Features and Set Temperature Symbology tools would enable more than one PPR file pair and ellipse layer to be processed at a time, requiring less supervision and input from the user, and thus saving time from the user perspective. It also may be beneficial to the user to have the option of declaring a filename for the output shapefiles/feature classes through the Create Features tool interface. Currently, the processed shapefiles/feature classes automatically inherit the base filename of the input PPR text file pair, with “_poly” or “_pt” added to the filename to indicate the respective ellipse or center point features.

The third potential, and most important, improvement to the toolkit would be a new tool to average Io’s surface temperature values for overlapping and proximate ellipses. Many regions consist of a multitude of overlapping IFOV ellipses, making it nearly impossible to visually discern approximate temperature values at these locations. Therefore, it would be helpful to scientists if only one temperature value is assigned to any single location. One possible approach might be to incorporate the functionality of the ArcGIS Polygon to Raster tool into the new custom tool, in order to convert the ellipses to a rasterized surface of temperature values. However, the fact that smaller IFOV ellipses yield more accurate temperature scans needs to be taken into account. The challenge would be to assign a higher weight to smaller ellipses, as opposed to larger overlapping IFOV ellipses, in determining temperature values. Next, a variety of spatial statistics could be run on the resulting surface temperature raster.

The custom toolkit could also be adapted to process PPR data for Jupiter and its Europa, Ganymede, and Callisto moons, shedding more light on temperature processes occurring on these planetary bodies.

Ultimately, client and user goals have been met. The Io PPR Data Processing Toolkit efficiently processes surface temperature data of Jupiter's moon Io for visualization in a GIS, thereby helping the client and her staff to meet the July 2011 USGS deadline for completion of a GIS containing the entire set of PPR data for Io. Such a GIS will help scientists and researchers to discern geospatial patterns in the surface temperatures of Io. With future integration of Galileo’s Near-Infrared Mapping Spectrometer and Solid State Imager data, the GIS could eventually be used to determine 
geographic areas of new and old eruptions on Io, and aid in analyzing corresponding surface composition. Future studies, combining data from the Galileo mission with data from the recent New Horizons flyby and previous Voyager and Cassini missions, could create a more complete timeline of volcanic activity on Io. 


\section{Works Cited}

Aronoff, S. (2005). Remote Sensing for GIS Managers. Redlands, CA: ESRI Press.

Baalke, R. (n.d.). The Discovery of the Galilean Satellites. Retrieved September 18, 2009, from Galileo: Journey to Jupiter: http://www2.jpl.nasa.gov/galileo/ganymede/discovery.html

Barrett, S. E. (2006a). Mapping the Amirani Region of Jupiter's Moon Io Using Multispectral Imagery and GIS. Redlands, CA: University of Redlands.

Barrett, S. E. (2006b). Documentation for Mapping the Amirani Region of Jupiter's Moon Io Using Multispectral Imagery and GIS [Data files]. Redlands, CA: University of Redlands.

Becker, T., \& Geissler, P. E. (2005, March). Galileo Global Color Mosaics of Io. Lunar and Planetary Science Conference, XXXVI, Abstract 1862.

Brewer, C. A. (2002). ColorBrewer 10-class diverging spectral. Retrieved July 30, 2009, from ColorBrewer - Selecting Good Color Schemes for Maps: http://www.personal.psu.edu/cab38/ColorBrewer/ColorBrewer.html

Carr, M. H., Masursky, H., Strom, R. G., \& Terrile, R. J. (1979). Volcanic features of Io. Nature, 280, 729-733.

Carr, M. H., Saunders, R. S., Strom, R. G., \& Wilhelms, D. E. (1984). The Geology of the Terrestrial Planets (NASA SP-469). Menlo Park, CA: United States Geological Survey.

Carr, M. H., Wilhelms, D. E., Greeley, R., \& Guest, J. E. (1976). Stratigraphy and structural geology. In R. Greeley, \& M. H. Carr (Eds.), A Geological Basis for the Exploration of the Planets (NASA SP-417) (pp. 13-32). Menlo Park, CA: United States Geological Survey.

Colwell, R. N. (1997). History and Place of Photographic Interpretation. In W. R. Philipson (Ed.), Manual of Photographic Interpretation (pp. 33 - 48). Bethesda, MD: American Society for Photogrammetry and Remote Sensing.

Davies, A. G. (2007). Volcanism on Io: A Comparison with Earth. New York: Cambridge University Press.

Davies, A. G., Keszthelyi, L. P., Williams, D. A., Phillips, C. B., McEwen, A. S., Lopes, R. M., et al. (2001). Thermal signature, eruption style, and eruption evolution at Pele and Pillan on Io. Journal of Geophysical Research , 106, 33079-33103.

Environmental Systems Research Institute. (n.d.). ArcInfo System Requirements. Retrieved September 10, 2009, from http://www.esri.com/software/arcgis/arcinfo/system-requirements.html 
Environmental Systems Research Institute. (2008). Types of geodatabases [Help sheet]. Retrieved June 16, 2009, from http://webhelp.esri.com/arcgisdesktop/9.3/index.cfm?TopicName=Types_of_geod atabases

Fischer, D. (2001). Mission Jupiter: The Spectacular Journey of the Galileo Spacecraft. New York: Springer.

Ghilani, C. D., \& Wolf, P. R. (2008). Elementary Surveying: An Introduction to Geomatics (12th ed.). Upper Saddle River, NJ: Pearson Higher Education.

Hanel, R., Conrath, B., Flasar, M., Kunde, V., Lowman, P., Maguire, W., et al. (1979). Infrared Observations of the Jovian System from Voyager 1. Science, 204 (4396), 972-976.

Hansen, J. E. (n.d.). PPR - Photopolarimeter-Radiometer. Retrieved June 22, 2009, from Galileo's Science Instruments: http://www2.jpl.nasa.gov/galileo/instruments/ppr.html

Hansen, V. L. (2000). Geologic mapping of tectonic planets. Earth and Planetary Science Letters, 176, 527-542.

Hare, T. M., Skinner, J. A., Tanaka, K. L., Fortezzo, C. M., Bleamaster, L. F., \& Sucharski, R. M. (2009, March). GIS-Based Planetary Geologic Maps: Recommendations for Improved Preparation, Review, and Publication. Retrieved September 29, 2009, from Planetary Data Systems, Techniques, and Interpretation; symposium conducted at the 40th annual meeting of the Lunar and Planetary Institute: http://www.lpi.usra.edu/meetings/lpsc2009/pdf/2538.pdf

Hare, T. M., Tanaka, K. L., \& Skinner, J. A. (2003, April). GIS 101 for Planetary Research. Retrieved September 29, 2009, from Advances in Planetary Mapping, Extraterrestrial Mapping Workshop; symposium conducted at the 34th annual meeting of the International Society for Photogrammetry and Remote Sensing: http://astrogeology.usgs.gov/Projects/ISPRS/MEETINGS/Houston2003/abstracts/ Hare_isprs_mar03.pdf

Keszthelyi, L., McEwen, A. S., Phillips, C. B., Milazzo, M., Geissler, P., Turtle, E. P., et al. (2001). Imaging of volcanic activity on Jupiter's moon Io by Galileo during the Galileo Europa Mission and the Galileo Millennium Mission. Journal of Geophysical Research, 106, 33025-33052.

Masursky, H., Schaber, G. G., Soderblom, L. A., \& Strom, R. G. (1979). Preliminary geological mapping of Io. Nature, 280, 725-729.

Matson, D. L., Blaney, D. L., Johnson, T. V., Veeder, G., \& Davis, A. (1998, March). Io and the Early Earth. Lunar and Planetary Science Conference XXIX, Abstract 1650. 
Morabito, L. A., Synnott, S. P., Kupferman, P. N., \& Collins, S. A. (1979). Discovery of Currently Active Extraterrestrial Volcanism. Science, 204 (4396), 972.

National Aeronautics and Space Administration. (n.d.). Basics of Space Flight, Section II: Chapter 12, Typical Science Instruments. Retrieved June 20, 2009, from http://www2.jpl.nasa.gov/basics/bsf12-1.php

National Aeronautics and Space Administration. (2001, October 1). The Journey to Jupiter: Introduction. Retrieved September 19, 2009, from Galileo Mission to Jupiter: http://www2.jpl.nasa.gov/galileo/mission/mission.html

National Aeronautics and Space Administration. (2007, August 9). Solar System Exploration: Galileo Legacy Site: Overview. Retrieved September 20, 2009, from http://solarsystem.nasa.gov/galileo/?CFID=22252556\&CFTOKEN=30615036

National Aeronautics and Space Administration. (2008). Solar System Exploration: Planets: Jupiter: Moons: Io [Fact sheet]. Retrieved July 27, 2009, from http://solarsystem.nasa.gov/planets/profile.cfm?Object=Jup_Io

Peale, S. J., Cassen, P., \& Reynolds, R. T. (1979). Melting of Io by Tidal Dissipation. Science, 203 (4383), 892-894.

Radebaugh, J., McEwen, A. S., Milazzo, M. P., Keszthelyi, L. P., Davies, A. G., Turtle, E. P., et al. (2004). Observations and temperatures of Io's Pele Patera from Cassini and Galileo spacecraft images. Icarus, 169, 65-79.

Rathbun, J. A., \& Barrett, S. E. (2007, March). Combining Galileo SSI, NIMS, and PPR data into GIS to study volcanic centers on Io. Lunar and Planetary Science Conference, XXXVIII, Abstract 2123.

Rathbun, J. A., Block, M. G., \& Spencer, J. R. (2005). Io from High Resolution Galileo PPR Data taken simultaneously with SSI or NIMS Observations. Lunar and Planetary Science Conference, XXXVI, Abstract 1990.

Rathbun, J. A., Spencer, J. R., Tamppari, L. K., Martin, T. Z., Barnard, L., \& Travis, L. D. (2004). Mapping of Io's thermal radiation by the Galileo photopolarimeterradiometer (PPR) instrument. Icarus, 169 (1), 127-139.

Schaber, G. G. (1980). The surface of Io: Geologic Units, morphology, and tectonics. Icarus, 43 (3), 302-333.

Schaber, G. G. (1982). The geology of Io. In D. Morrison (Ed.), Satellites of Jupiter (pp. 556-597). Tuscon, Arizona: University of Arizona Press.

Schaber, G. G. (2005). The U.S. Geological Survey, Branch of Astrogeology - A Chronology of Activities from Conception through the End of Project Apollo (1960-1973). U.S. Geological Survey Open-File Report 2005-1190. Retrieved 
September 29, 2009, from United States Geological Survey:

http://pubs.usgs.gov/of/2005/1190/

Smith, B. A., Soderblom, L. A., Beebe, R., Boyce, J., Briggs, G., Carr, M., et al. (1979a). The Galilean Satellites and Jupiter: Voyager 2 Imaging Science Results. Science, 206 (4421), 927-950.

Smith, B. A., Soderblom, L. A., Johnson, T. V., Ingersoll, A. P., Collins, S. A., Shoemaker, E. M., et al. (1979b). The Jupiter System through the Eyes of Voyager 1. Science, 204 (4396), 951-972.

Smythe, W. D., Lopes-Gautier, R., Ocampo, A., Hui, J., Segura, M., Soderblom, L. A., et al. (1995). Galilean satellite observation plans for the Near-Infrared Mapping Spectrometer experiment on the Galileo spacecraft. Journal of Geophysical Research, 100, 18957-18972.

Spencer, J. R., Rathbun, J. A., Travis, L. D., Tamppari, L. K., Barnard, L., Martin, T. Z., et al. (2000). Io's thermal emission from the Galileo PhotopolarimeterRadiometer. Science, 288 (5469), 1198-1201.

Stone, E. C., \& Lane, A. L. (1979a). Voyager 1 Encounter with the Jovian System. Science, 204 (4396), 945-948.

Stone, E. C., \& Lane, A. L. (1979b). Voyager 2 Encounter with the Jovian System. Science, 206 (4421), 925-927.

Tanaka, K. L. (1994). The Venus Geologic Mappers Handbook. U.S. Geological Survey Open-File Report 94-438. Retrieved September 29, 2009, from United States Geological Survey: http://astrogeology.usgs.gov/Projects/PlanetaryMapping/VenusMappers/VGM_H andbook_94.pdf

The Columbia Electronic Encyclopedia. (2005). Planetary Science. Retrieved May 17, 2009, from http://encyclopedia2.thefreedictionary.com/planetary+science

Travis, L. D., Martin, T. Z., Orton, G. S., Spencer, J. R., Tamppari, L. K., Beebe, R. F., et al. (2002). GO-J-PPR-3-RDR-V1.0 [Data set and metadata]. Retrieved September 29, 2009, from NASA Planetary Data System Web site: http://starbrite.jpl.nasa.gov/pds/viewProfile.jsp?dsid=GO-J-PPR-3-RDR-V1.0

United States Geological Survey. (2008). Jupiter Satellites: Io [Fact sheet and data files]. Retrieved June 17, 2009, from http://astrogeology.usgs.gov/Projects/JupiterSatellites/io.html

United States Geological Survey. (2009). Gazetteer of Planetary Nomenclature: Jovian System Images with Names: Io [Fact sheet]. Retrieved June 17, 2009, from http://planetarynames.wr.usgs.gov/images/io_comp_color.pdf 
Wilhelms, D. E. (1990). Geologic mapping. In R. Greeley (Ed.), Planetary Mapping (pp. 208-260). New York: Cambridge University Press.

Williams, D. A., Greeley, R., McEwen, A., Keszthelyi, L., Geissler, P., Radebaugh, J., et al. (2002, October). High-temperature Ultrabasic Eruptions on Jupiter's Moon Io: Latest Results from the Galileo Mission and Assessment of Eruption Styles. Retrieved September 29, 2009, from There and Back Again: Terrestrial Approaches to Extraterrestrial Problems; symposium conducted at the annual meeting of the Geological Survey of America:

http://gsa.confex.com/gsa/2002AM/finalprogram/abstract_37696.htm 



\section{Appendix A. Project Plan}

\begin{tabular}{|c|c|c|c|c|c|c|c|c|}
\hline \multirow{2}{*}{$\begin{array}{c}\text { Phase/ } \\
\text { Task }\end{array}$} & \multirow[b]{2}{*}{ Description } & \multicolumn{7}{|c|}{$2008-2009$} \\
\hline & & Oct & Nov & Dec & Jan & Feb & Mar & Ap \\
\hline Phase 1 & Requirements Analysis \& Development & & & & & & & \\
\hline 1.1 & Needs Assessment & & & & & & & \\
\hline 1.2 & Project Plan & & & & & & & \\
\hline 1.3 & Draft Needs Assessment \& Project Plan Documents & & & & & & & \\
\hline \multirow[t]{2}{*}{1.4} & Final Preliminary Needs Assessment \& Project Plan Documents & & & 8 & & & & \\
\hline & Needs Assessment \& Project Plan Complete & & & 0 & & & & \\
\hline Phase 2 & Data Download \& Exploration & & & & & & & \\
\hline 2.1 & Download all data & & & & & & & \\
\hline 2.2 & Plot and project coordin ate point in ArcMap & & & & & & & \\
\hline \multirow[t]{2}{*}{2.3} & Explore data in ArcMap & & & & & & & \\
\hline & Data Download \& Exploration Complete & & & & & & & \\
\hline Phase 3 & Geodata base Design \& Implementation & & & & & & & \\
\hline 4.1 & Conceptual Design & & & & & & & \\
\hline 4.2 & Physical Design & & & & & & & \\
\hline 4.3 & Implementation & & & & & & & \\
\hline \multirow[t]{2}{*}{4.4} & Prototype File Geodatabase Complete & & & & & & & 0 \\
\hline & Geodatabase Design \& Construction Complete & & & & & & & 0 \\
\hline
\end{tabular}

\begin{tabular}{|c|c|c|c|c|c|c|c|}
\hline \multirow{2}{*}{$\begin{array}{c}\text { Phase/ } \\
\text { Task }\end{array}$} & \multirow[b]{2}{*}{ Description } & \multicolumn{6}{|c|}{$2008-2009$} \\
\hline & & May & Jun & Jul & Aug & Sep & Oct \\
\hline Phase 4 & Prototype Development \& Testing & & & & & & \\
\hline 5.1 & Conceptual Design & & & & $\cdot 1$ & & \\
\hline 5.2 & Physical Design & & & & $\bullet$ & & \\
\hline 5.3 & Programming (Implementation \& Testing): Tool Interface/Functions & & & & $\cdot 8$ & & \\
\hline 5.4 & Prototype ArcGIS Toolkit Complete & & & 0 & $\cdot$ & & \\
\hline \multirow[t]{2}{*}{5.5} & Preliminary ArcGIS Toolkit Complete & & & & 0.1 & & \\
\hline & Prototype Development \& Testing Complete & & & & 0. & & \\
\hline Phase 5 & ESRI User Conference, MIP Defense, MIP Report, \& Documentation & & & & & & \\
\hline 6.1 & Powerpoint \& Poster for ESRI User Conference & & & & 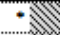 & & \\
\hline 6.2 & ESRI User Confer ence Presentat ion & & & 0 & $\cdot 1$ & & \\
\hline 6.3 & $\begin{array}{l}\text { Draft chapters of written report \& edits } \\
\text { - }\end{array}$ & & & & 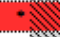 & & \\
\hline 6.4 & Draft Prototype ArcGIS toolkit to Dr. Rathbun (Client) & & & & $\cdot$ & & \\
\hline 6.5 & Defense Prep aration & & & & 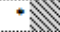 & & \\
\hline 6.6 & Defense & & & & $\cdot$. & 0 & \\
\hline 6.7 & CD/DVD Preparation of all data and ArcG IS files & & & & -... & & \\
\hline 6.8 & Final written report, poster; CD/DVD of all data and ArcGIS files to MS GIS Program & & & & $\cdot-1$ & & 0 \\
\hline \multirow[t]{3}{*}{6.9} & Final Prototype ArcG IS toolkit \& documentation to Dr. Rathbun (Client) & & & & -... & & 0 \\
\hline & Valediction! & & & & 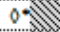 & & \\
\hline & Graduation! & & & & $\cdot$ & & 0 \\
\hline
\end{tabular}





\section{Appendix B. Visual Basic Code for Io PPR Data Processing Toolkit}

\section{B.1 Code for Create Features Dialog Box}

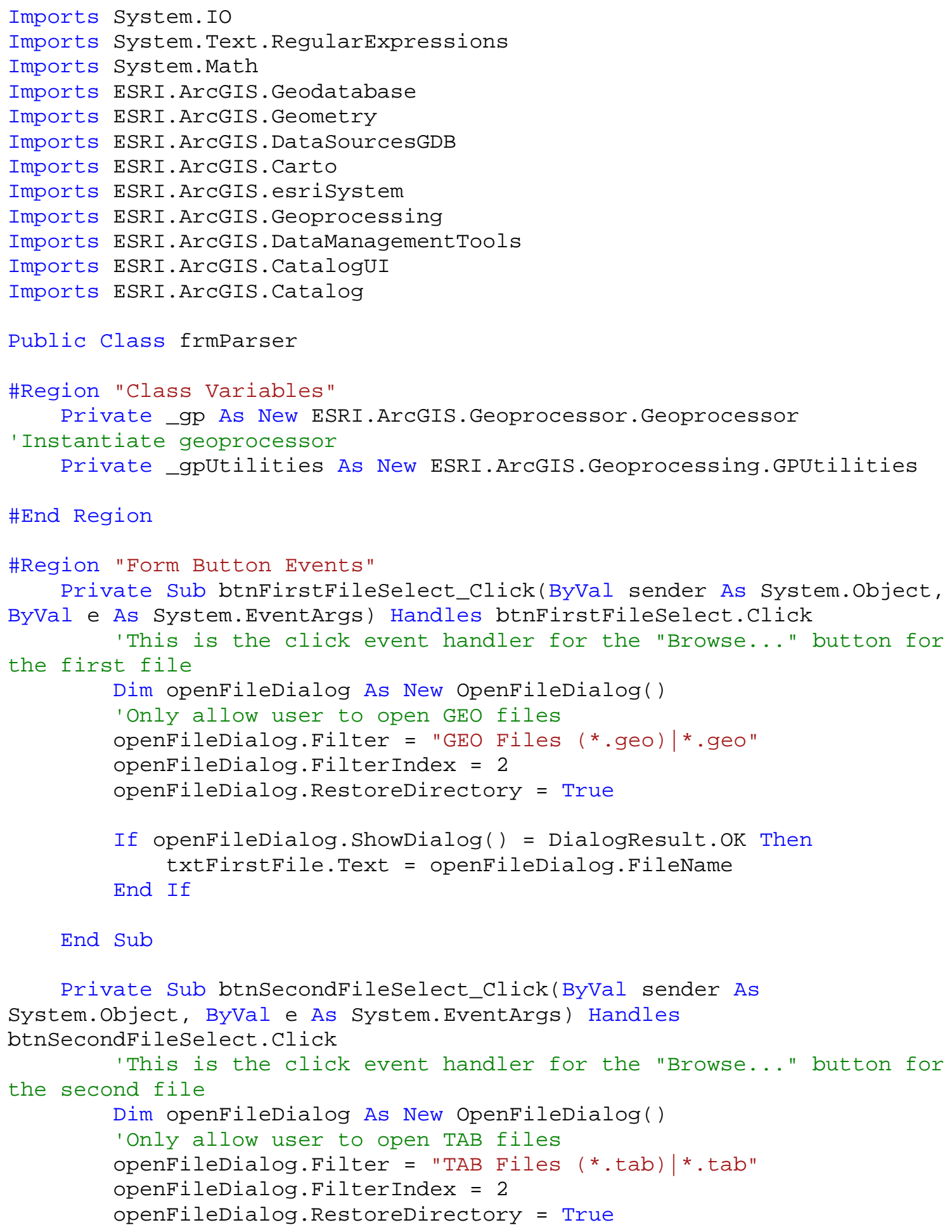

End Sub

Private Sub btnSecondFileSelect_Click(ByVal sender As System.object, ByVal e As System.EventArgs) Handles btnSecondFileSelect. Click

'This is the click event handler for the "Browse..." button for the second file

Dim openFiledialog As New OpenFileDialog()

'Only allow user to open TAB files

openFileDialog.Filter = "TAB Files (*.tab)|*.tab"

openFileDialog. FilterIndex $=2$

openFileDialog. RestoreDirectory = True 
If openFileDialog.ShowDialog ()$=$ DialogResult.OK Then

End If

txtSecondFile.Text $=$ openFileDialog.FileName

End Sub

Private Sub btnSelectWorkspace_Click(ByVal sender As System.object,

Byval e As System.EventArgs) Handles btnSelectWorkspace.Click

Dim workspaceFilter As IGxobjectFilter

workspaceFilter = New GxFilterContainers

Dim pEnumgx As IEnumGXobject

Dim arcGISDialog As IGXDialog = New GXDialog

arcGISDialog. AllowMultiselect $=$ False

arcGISDialog.ButtonCaption = "Select"

arcGISDialog.Title = "Select output workspace"

arcGISDialog.objectFilter = workspaceFilter

If Not arcGISDialog.DoModalopen( $\odot$, pEnumGX) Then

End If

Exit Sub 'Exit if user presses Cancel

txtOutputWorkspace. Text $=$ pEnumgx. Next. FullName

End Sub

Private Sub btnParse_Click(ByVal sender As System.object, ByVal e As System.EventArgs) Handles btnParse.Click

buttton

'This is the click event handler for the "Create Features"

'All the necessary parsing and array creation are coded here textboxes

'Verify that user has entered something into all required

If txtFirstFile.Text. Length $<1$ or txtSecondFile.Text. Length $<$

1 or txtoutputWorkspace. Text. Length $<1$ Then MsgBox("Please fill in all information before continuing.", MsgBoxStyle.Exclamation, "Error")

End If Exit Sub

Windows. Forms. Cursor. Current $=$ Cursors. WaitCursor

If Not (System.IO.File.Exists(txtFirstFile.Text)) Then

\& vbCrLf \&

MsgBox("The .GEO filepath does not exist or is incomplete."

correct.", MsgBoxStyle.Exclamation, -

End If

Exit Sub

"File Error")

If Not

(txtFirstFile.Text.Substring(txtFirstFile.Text . LastIndexof(" . ")). ToLowe $r=$ ".geo") Then

MsgBox("Please select a valid.GEO file.",

MsgBoxStyle.Exclamation, -

"File Error")

End If

Exit Sub

If Not (System.IO.File.Exists(txtSecondFile.Text)) Then

\& vbCrLf \&

MsgBox("The .TAB filepath does not exist or is incomplete." 


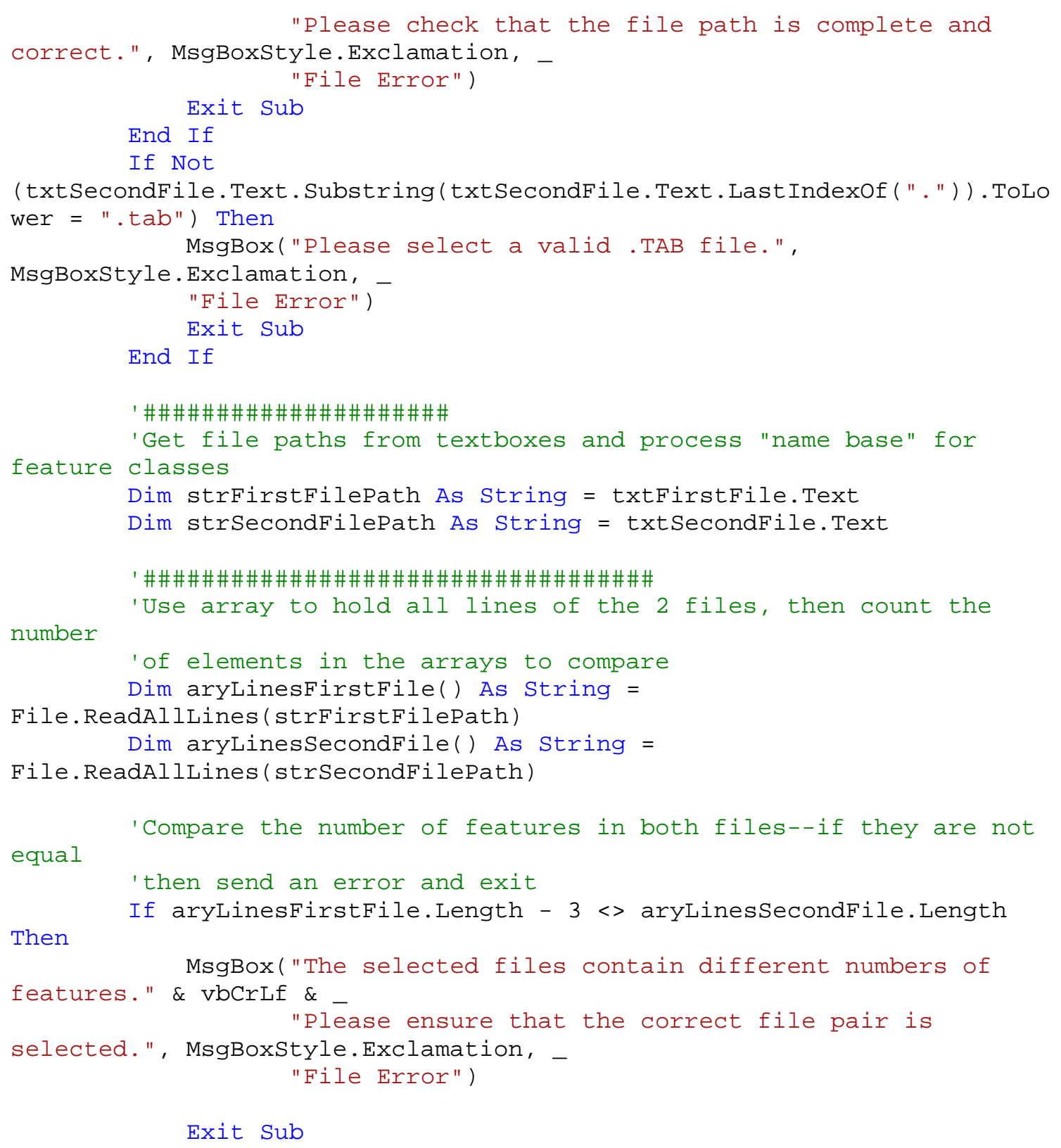


If Not workspace.Contains(".sde") And Not

workspace.Contains(".gdb") And Not workspace.Contains(".mdb") Then

End If pointPath = pointPath + ".shp" polyPath = polyPath + ".shp"

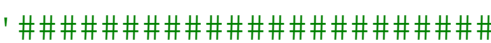

'Make the empty point and poly feature classes using MakeFeaturePointandPolyFCs( ) False Then

If MakeFeaturePointandPolyFCs(workspace, nameBaseForClasses) = cancelled.", MsgBoxStyle.Exclamation, -

End If Exit Sub "Error")

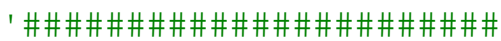

'Get a reference to the Feature Classes from the geoprocessor Dim pointFC As IFeatureClass = _gputilities.OpenFeatureClassFromString(pointPath) Dim polyFC As IFeatureclass = _gpUtilities.OpenFeatureclassFromstring(polyPath)

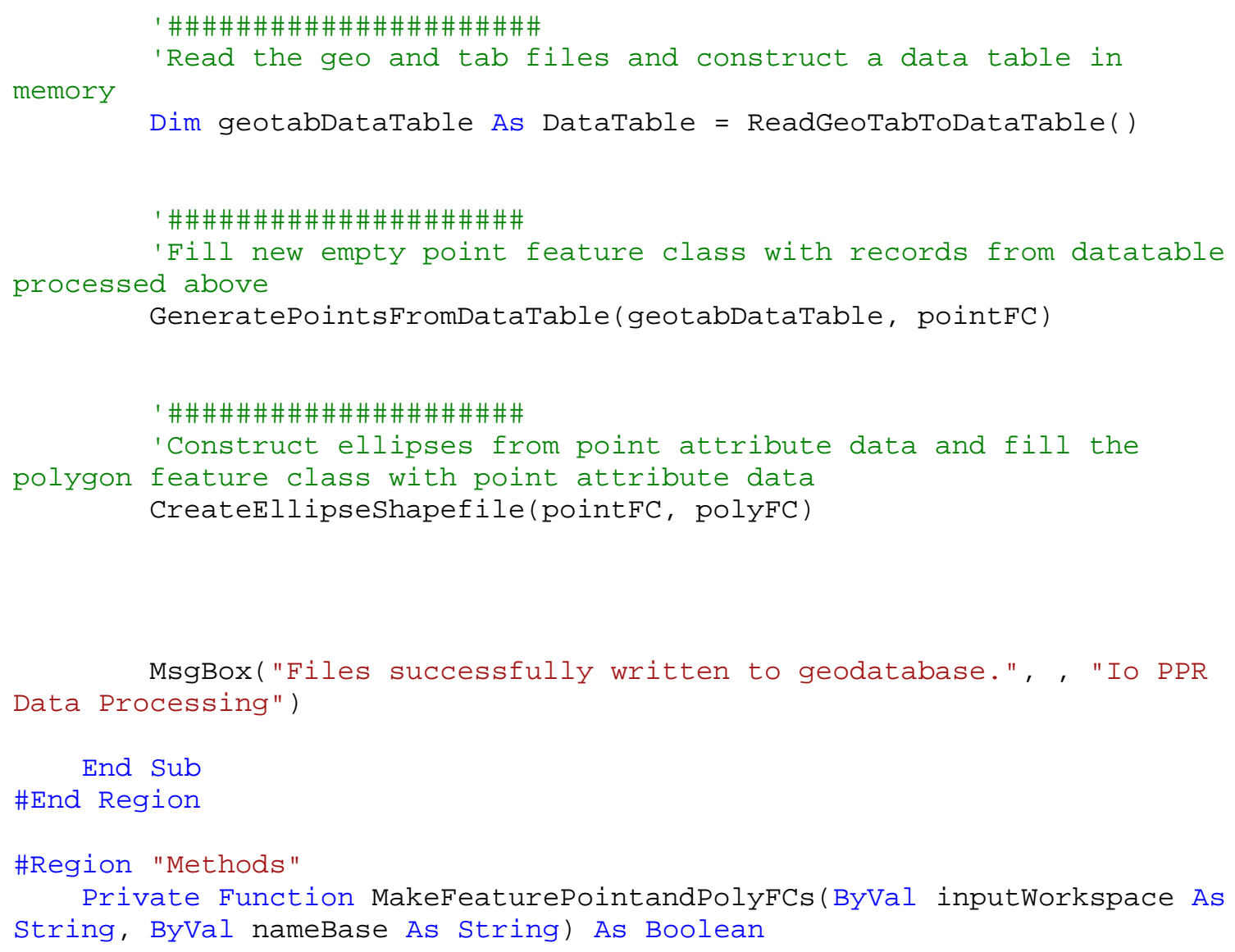


Dim pointName As String = nameBase \& "_pt"

Dim polyName As String = nameBase \& "_poly"

If Not inputWorkspace.Contains(".sde") And Not inputWorkspace.Contains(".gdb") And Not inputWorkspace.Contains(".mdb") Then

End If

pointName $=$ pointName + ".shp" polyName $=$ polyName + ".shp"

Dim pointPath As String = inputWorkspace \& "\" \& pointName

Dim polyPath As String = inputWorkspace \& "\" \& polyName

Dim createPointFeatureclass As New

CreateFeatureclass (inputWorkspace, pointName)

createPointFeatureClass.geometry_type = "POINT"

createPointFeatureclass. spatial_reference $=$

"GEOGCS['GCS_Io_2000' ', DATUM[' D_Io_2000', SPHEROID [ ' Io_2000_IAU_IAG' , 1821

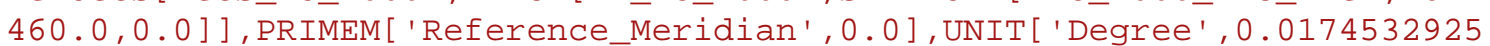
$199433]$ ]"

_gp.Execute(createPointFeatureclass, Nothing)

'Add all the fields for the point feature class

Dim addFieldTool As New AddField

addFieldTool = New AddField(pointPath, "ADJ_SCLK", "TEXT")

-gp.Execute(addFieldTool, Nothing)

addFieldTool = New AddField (pointPath, "LAT", "DOUBLE")

_gp.Execute(addFieldTool, Nothing)

addFieldTool = New AddField (pointPath, "LON", "DOUBLE")

_gp.Execute(addFieldTool, Nothing)

addFieldTool = New AddField (pointPath, "NEWLON", "DOUBLE")

_gp.Execute(addFieldTool, Nothing)

addFieldTool = New AddField (pointPath, "SIPDIS", "DOUBLE")

_gp. Execute (addFieldTool, Nothing)

addFieldTool = New AddField(pointPath, "SSCLON", "DOUBLE")

_gp.Execute(addFieldTool, Nothing)

addFieldTool = New AddField (pointPath, "NEWSSCLON", "DOUBLE")

_gp.Execute(addFieldTool, Nothing)

addFieldTool = New AddField(pointPath, "SSCLAT", "DOUBLE")

_gp.Execute(addFieldTool, Nothing)

addFieldTool = New AddField (pointPath, "SMIN_KM", "DOUBLE")

-gp.Execute(addFieldTool, Nothing)

addFieldTool = New AddField (pointPath, "SMIN_AD", "DOUBLE")

_gp. Execute (addFieldTool, Nothing)

addFieldTool = New AddField (pointPath, "SMIN_DD", "DOUBLE")

-gp.Execute(addFieldTool, Nothing)

addFieldTool = New AddField (pointPath, "SMAJ_KM", "DOUBLE")

_gp.Execute(addFieldTool, Nothing)

addFieldTool $=$ New AddField (pointPath, "SMAJ_AD", "DOUBLE")

-gp.Execute(addFieldTool, Nothing)

addFieldTool = New AddField(pointPath, "SMAJ_DD", "DOUBLE")

_gp.Execute(addFieldTool, Nothing)

addFieldTool = New AddField (pointPath, "MINMAJ_RAT", "DOUBLE")

_gp.Execute(addFieldTool, Nothing)

addFieldTool = New AddField(pointPath, "ROT_ANGLE", "DOUBLE")

_gp.Execute(addFieldTool, Nothing)

addFieldTool = New AddField(pointPath, "TEMP", "DOUBLE")

_gp.Execute(addFieldTool, Nothing) 
Dim createPolygonFeatureclass As New

CreateFeatureclass(inputWorkspace, polyName)

createPolygonFeatureclass.geometry_type = "POLYGON"

createPolygonFeatureclass.spatial_reference $=$

"GEOGCS['GCS_Io_2000 ' , DATUM [ 'D_Io_2000 ' , SPHEROID [ ' Io_2000_IAU_IAG ' , 1821

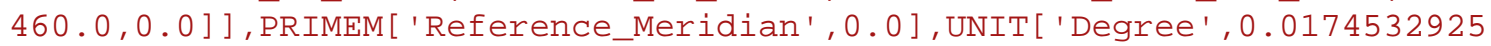
199433] ]"

_gp.Execute(createPolygonFeatureclass, Nothing)

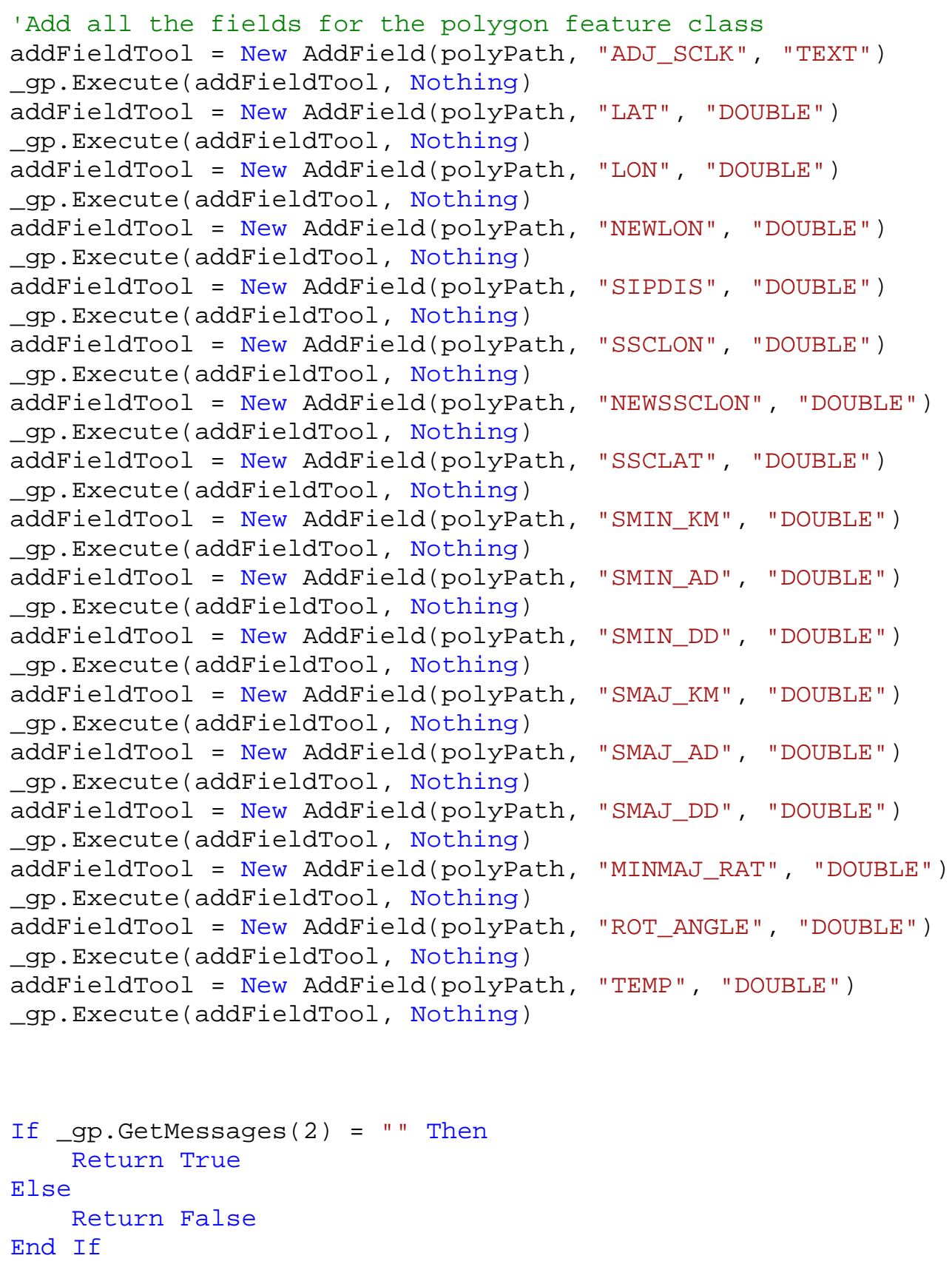


'\#\#\#\#Create Empty DataTable and add all columns

Dim geotabTable As DataTable = New DataTable

Dim myCol As DataColumn

myCol = New DataColumn("ADJ_SCLK",

System.Type.GetType("System.String"))

geotabTable.Columns. Add (myCol)

myCol = New DataColumn ("LAT",

System. Type.GetType("System. Decimal"))

geotabTable.Columns. Add (myCol)

myCol = New DataColumn("LON",

System.Type.GetType("System.Decimal"))

geotabTable.Columns. Add (myCol)

myCol = New DataColumn("NEWLON",

System. Type.GetType("System. Decimal"))

geotabTable.Columns. Add (myCol)

myCol = New DataColumn("SIPDIS",

System.Type.GetType("System. Decimal"))

geotabTable. Columns. Add (mycol)

myCol = New DataColumn("SSCLON",

System. Type.GetType("System. Decimal"))

geotabTable. Columns. Add (myCol)

myCol = New DataColumn("NEWSSCLON",

System. Type.GetType("System. Decimal")) geotabTable.Columns. Add (myCol) myCol = New DataColumn("SSCLAT",

System. Type.GetType("System. Decimal")) geotabTable.Columns. Add (myCol) myCol = New DataColumn("SMIN_KM",

System. Type.GetType("System. Decimal")) geotabTable.Columns. Add (myCol) myCol = New DataColumn ("SMIN_AD",

System.Type.GetType("System. Decimal")) geotabTable.Columns. Add (myCol) myCol = New DataColumn("SMIN_DD",

System. Type.GetType("System. Decimal")) geotabTable.Columns. Add (myCol) myCol = New DataColumn ("SMAJ_KM",

System. Type.GetType("System. Decimal")) geotabTable.Columns. Add (myCol) myCol = New DataColumn("SMAJ_AD",

System. Type.GetType("System. Decimal")) geotabTable.Columns. Add (myCol) myCol = New DataColumn ("SMAJ_DD",

System. Type.GetType("System. Decimal")) geotabTable.Columns. Add (myCol) myCol = New DataColumn("MINMAJ_RAT",

System. Type.GetType("System. Decimal")) geotabTable. Columns. Add (myCol) myCol = New DataColumn("ROT_ANGLE",

System. Type.GetType("System. Decimal")) geotabTable.Columns. Add (myCol) myCol = New DataColumn("TEMP",

System. Type.GetType("System. Decimal")) geotabTable. Columns. Add (myCol)

Dim strFirstFilePath As String = txtFirstFile. Text

Dim strSecondFilePath As String $=$ txtSecondFile.Text 


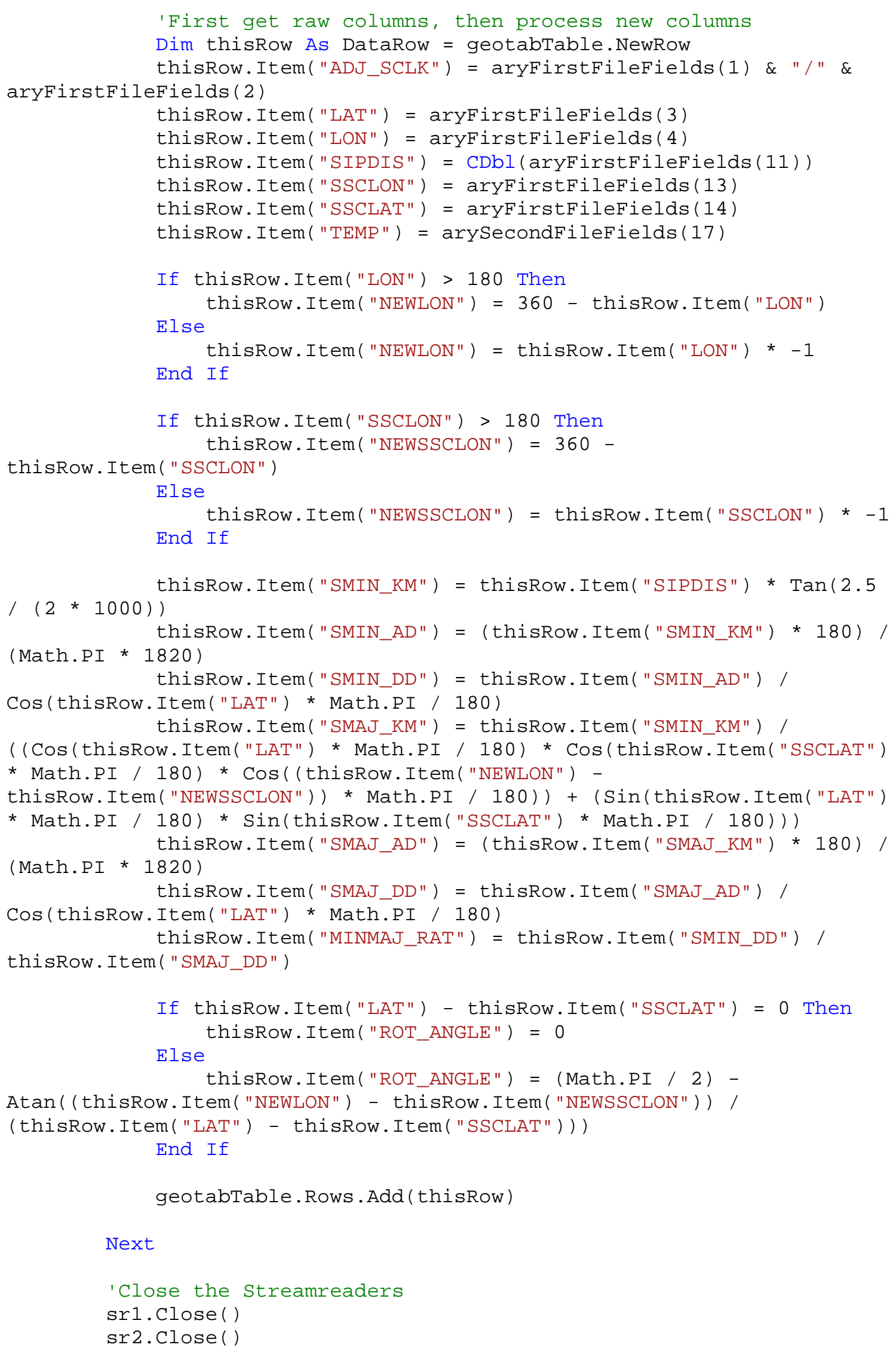


Return geotabTable

GC. Collect ( )

End Function

Private Sub GeneratePointsFromDataTable(ByVal geotabDataTable As DataTable, ByVal pointFC As IFeatureClass) Try

For Each currentRow As DataRow In geotabDataTable. Rows

'Create the feature and its geometry

Dim newFeature As IFeature = pointFC.CreateFeature ()

Dim point As Point $=$ New Point

point $. X=$ currentRow ("NEWLON")

point.Y = currentRow( "LAT")

newFeature. Shape $=$ point

'Add all the attribute values

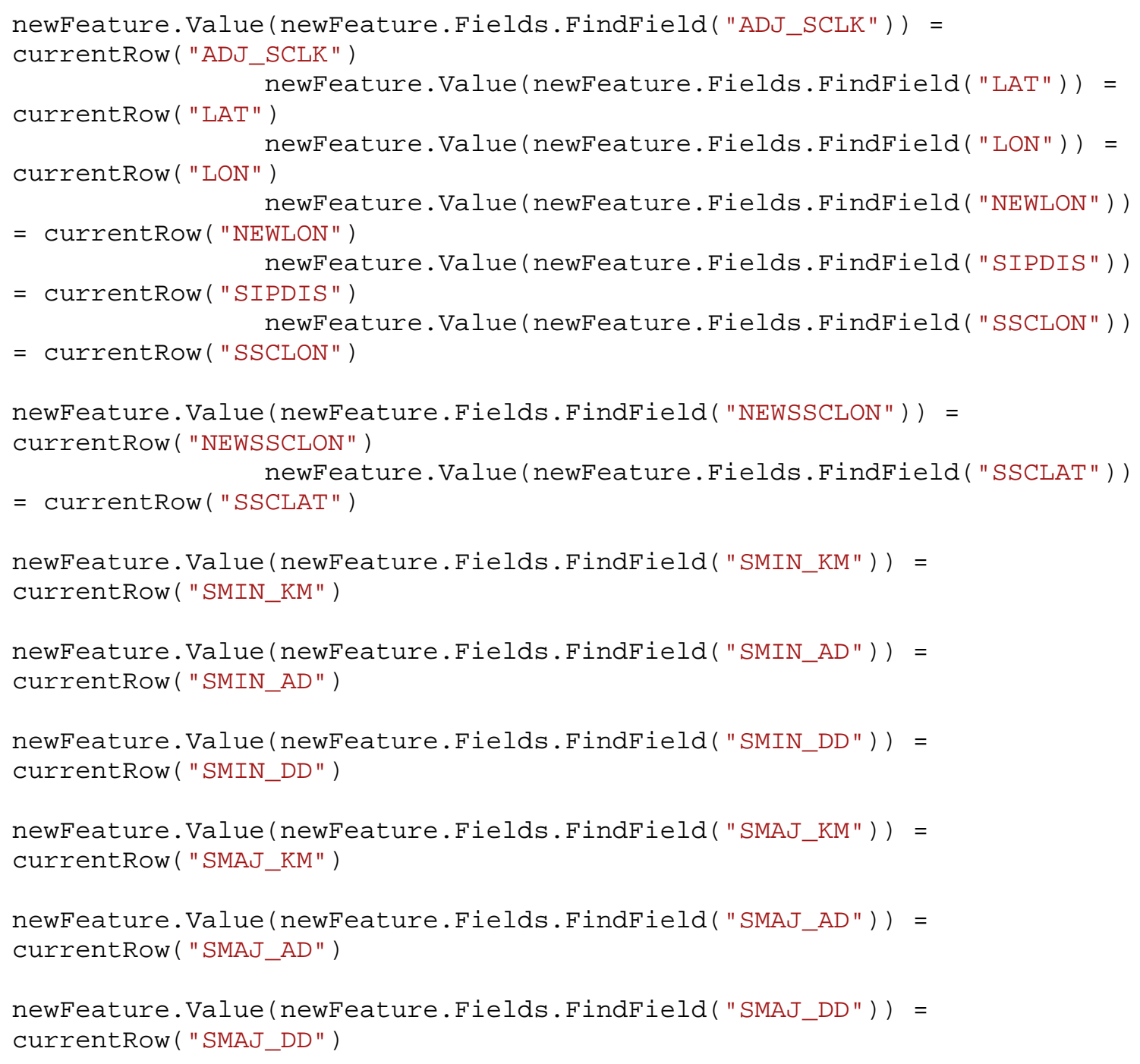




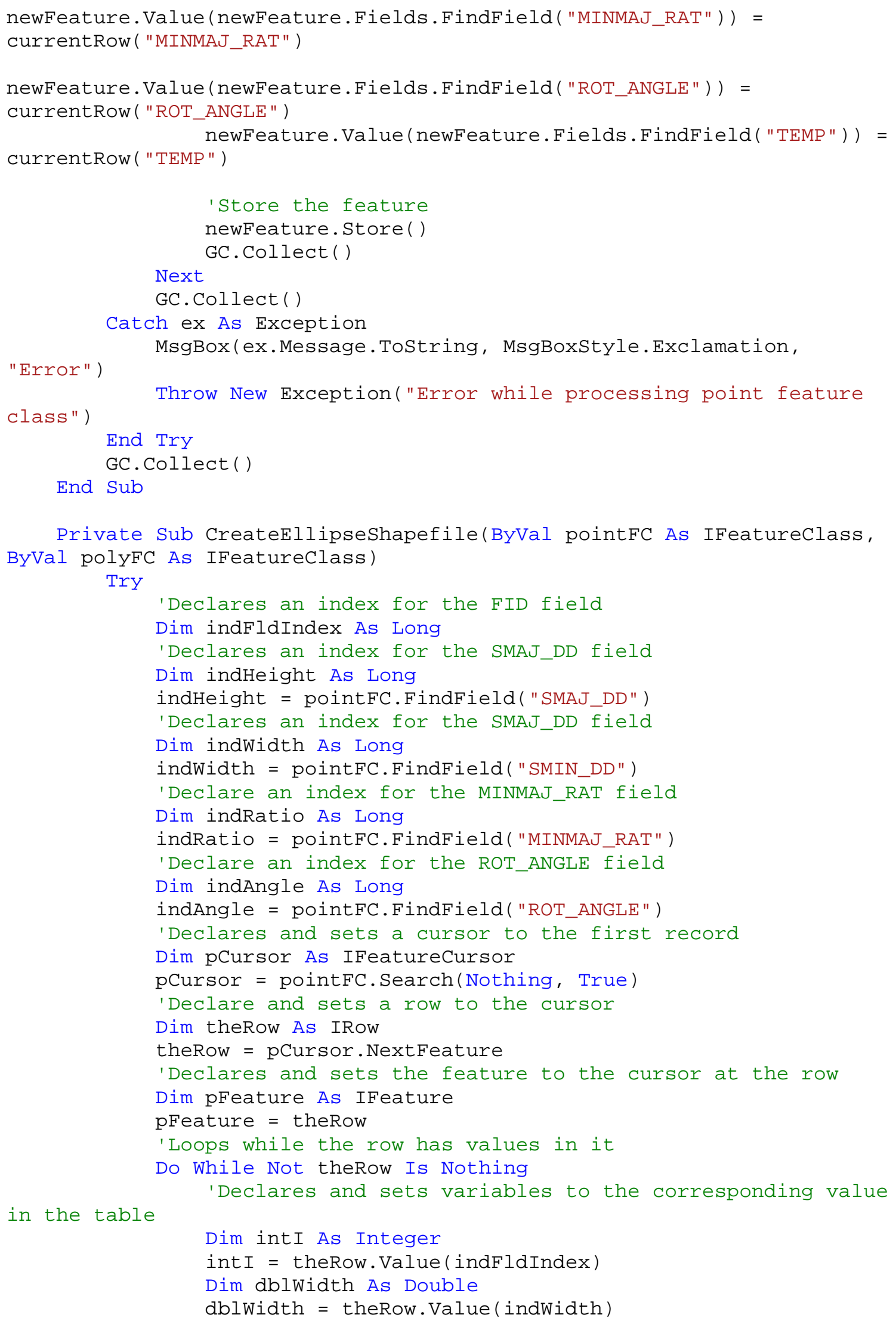


Dim dblHeight As Double

dblHeight $=$ theRow.Value(indHeight)

Dim dblRatio As Double

dblRatio = theRow.Value (indRatio)

Dim dblAngle As Double

$\mathrm{dblAngle}=$ theRow . Value (indAngle)

'Declare Ellipse Variables

Dim pPolygon As IPolygon

Dim pConstructEllipticArc As IConstructEllipticArc

Dim pEllipticArc As IEllipticArc

Dim pSeg As ISegment

Dim pSegcoll As ISegmentCollection

Dim pRing As IRing

Dim pGeomColl As IGeometryCollection

Dim pEnv As IEnvelope

Dim pSR As ISpatialReference

pSR $=$ Nothing

'Declare and set point feature to current feature

Dim pPoint As IPoint

pPoint = pFeature. Shape

'Determine extent of ellipse

'Set Envelope spatial reference to the current map's

'Create Ellipse Elements

pEllipticArc = New EllipticArc

pEllipticArc. SpatialReference $=$ pSR

pConstructEllipticArc $=$ pEllipticArc

'Draw ellipse from center point, from angle, central

angle, rotation angle, semi major axis, minor Major Ratio

pEllipticArc.PutCoordsByAngle(False, pPoint, 0,2 *

3.14159265358979, dblAngle, dblHeight, dblRatio)

'Draw Ellipse

pSeg = pEllipticArc

pSegcoll = New Ring

pSegcoll. AddSegment ( $p$ Seg)

pRing $=$ pSegcoll

pPolygon = New Polygon

pPolygon. SpatialReference $=$ pSR

pGeomColl = pPolygon

pGeomColl. AddGeometry (pRing)

'Create insert cursor

Dim pIFCursor As IFeatureCursor

pIFCursor $=$ polyFC. Insert (True)

'Create feature buffer

Dim pIFBuffer As IFeatureBuffer

pIFBuffer = polyFC.CreateFeatureBuffer

pIFBuffer. Shape = pPolygon

'pIFBuffer.Value(2) = intI

pIFBuffer.Value (pIFBuffer.Fields.FindField("ADJ_SCLK"))

$=$ theRow.Value (theRow.Fields.FindField("ADJ_SCLK"))

pIFBuffer.Value (pIFBuffer.Fields.FindField ("LAT")) =

theRow.Value (theRow. Fields. FindField("LAT"))

pIFBuffer.Value (pIFBuffer.Fields.FindField("LON")) =

theRow.Value (theRow. Fields. FindField("LON")) 
pIFBuffer.Value (pIFBuffer.Fields.FindField("NEWLON")) = theRow.Value (theRow. Fields.FindField("NEWLON"))

pIFBuffer.Value (pIFBuffer.Fields.FindField("SIPDIS")) = theRow.Value (theRow. Fields.FindField("SIPDIS")) pIFBuffer.Value (pIFBuffer.Fields.FindField ("SSCLON")) = theRow.Value (theRow. Fields. FindField("SSCLON"))

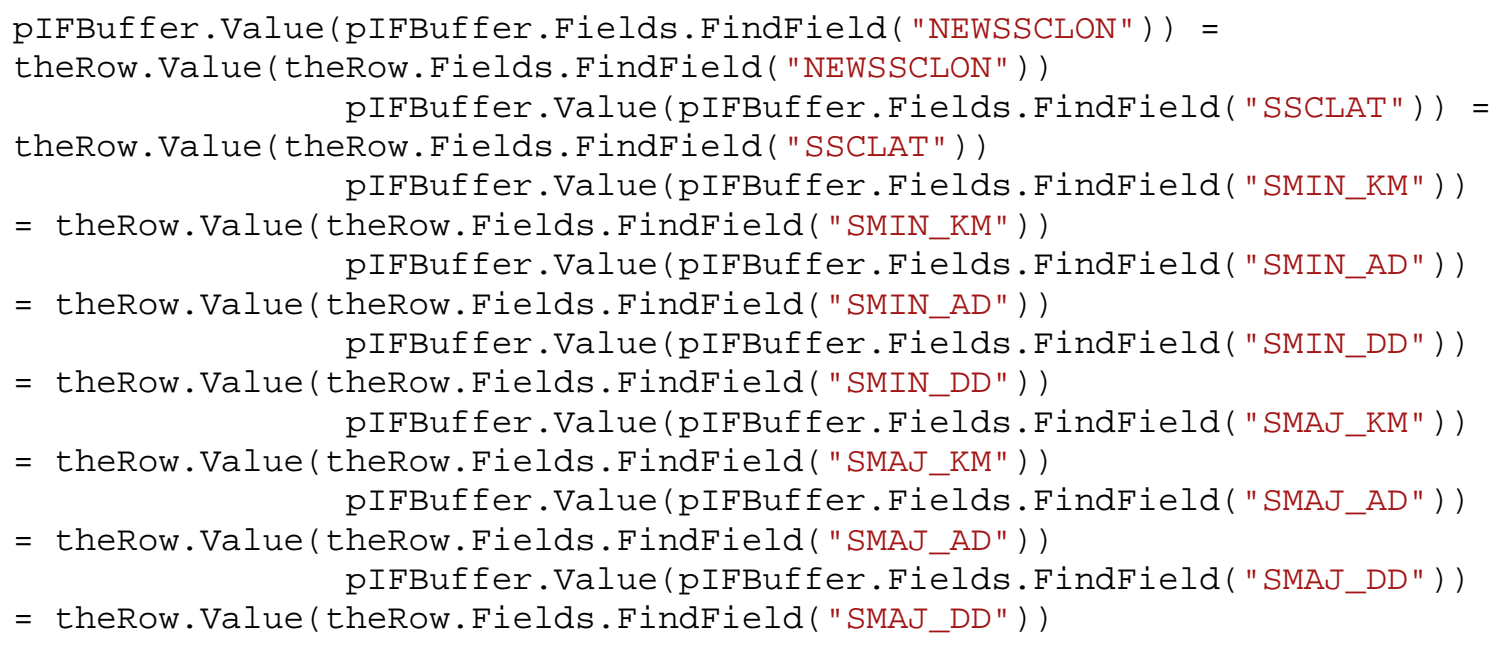


Private Sub txtFirstFile_Validating(ByVal sender As System.object, Byval e As System.ComponentModel.CancelEventArgs) Handles

txtFirstFile.Validating

If txtFirstFile.Text. Length $=0$ Then

End If

Exit Sub

If Not (System.IO.File.Exists(txtFirstFile.Text)) Then

MsgBox("The .GEO filepath does not exist or is incomplete."

\& vbCrLf \&

"Please check that the file path is complete and

correct.", MsgBoxstyle.Exclamation, -

End If

Exit Sub

"File Error")

If Not

(txtFirstFile.Text.Substring(txtFirstFile.Text . Last Indexof(" . ")) . ToLowe $r=" . g e o ")$ Then

MsgBox("Please select a valid. GEO file.",

MsgBoxStyle.Exclamation,

"File Error")

End If

Exit Sub

End Sub

Private Sub txtSecondFile_Validating(ByVal sender As System.object, ByVal e As System.ComponentModel.CancelEventArgs) Handles

txtSecondFile.Validating

If txtSecondFile.Text. Length $=\odot$ Then

End If Exit Sub

If Not (System.IO.File.Exists(txtSecondFile.Text)) Then

\& vbCrLf \&

MsgBox("The .TAB filepath does not exist or is incomplete."

correct.", MsgBoxstyle.Exclamation, -

End If

Exit Sub

"File Error")

If Not

(txtSecondFile.Text. Substring(txtSecondFile.Text . LastIndexof(" . ") ) . ToLo wer $=" \cdot$ tab") Then MsgBox("Please select a valid. TAB file.",

MsgBoxStyle.Exclamation, -

"File Error")

End If

Exit Sub

End Sub

End Class 


\section{B.2 Code for Create Features ArcMap Tool Button}

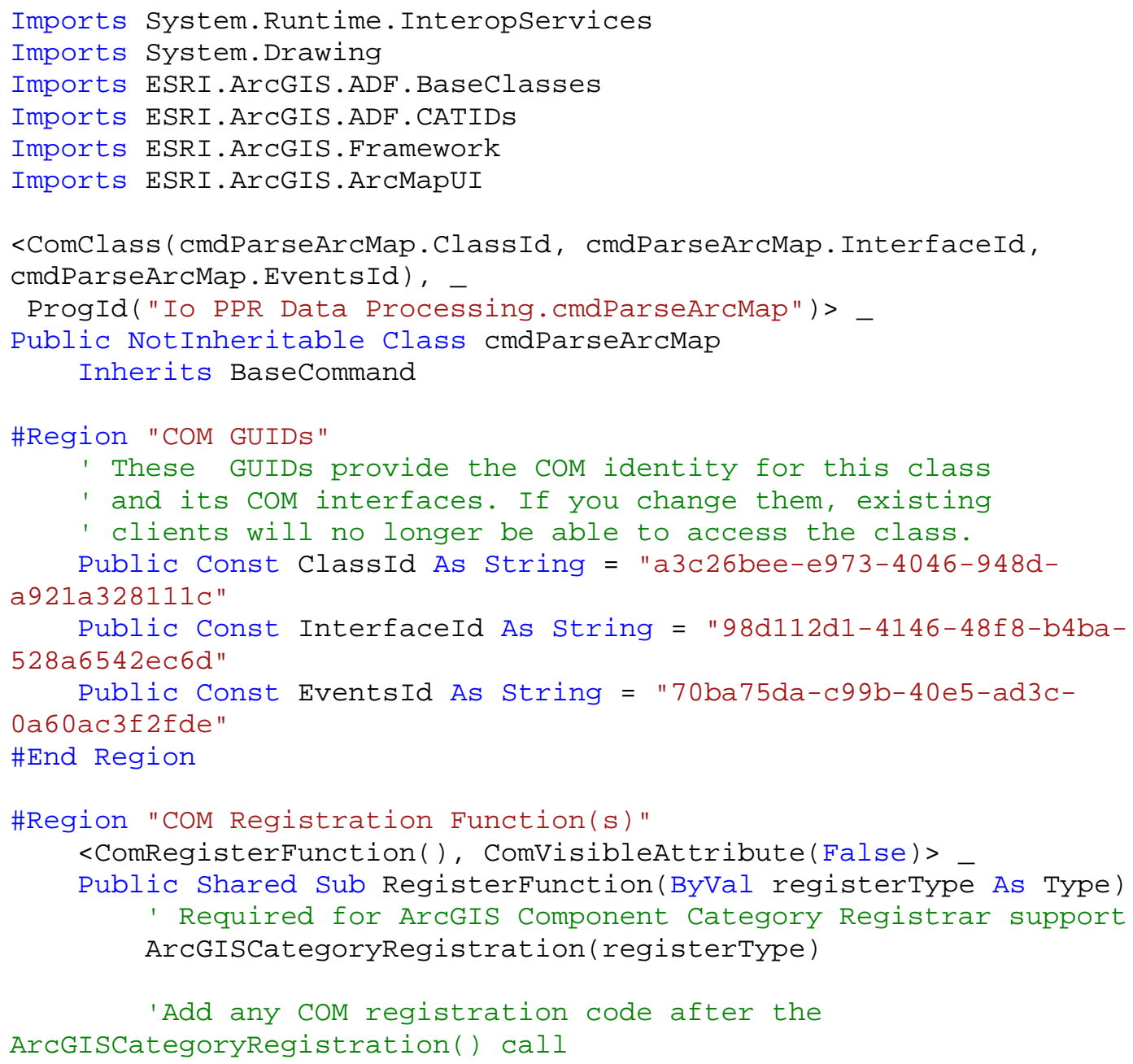

End Sub

<ComUnregisterFunction(), ComVisibleAttribute(False)>

Public Shared Sub UnregisterFunction(ByVal registerType As Type)

' Required for ArcGIS Component Category Registrar support ArcGISCategoryUnregistration(registerType)

'Add any COM unregistration code after the ArcGISCategoryUnregistration() call

End Sub

\#Region "ArcGIS Component Category Registrar generated code"

Private Shared Sub ArcGISCategoryRegistration(ByVal registerType As Type )

Dim regkey As String =

String.Format("HKEY_CLASSES_ROOT\CLSID $\{\{\{0\}\}\} "$, registerType.GUID)

MxCommands.Register (regKey)

End Sub 
Private Shared Sub ArCGISCategoryUnregistration(ByVal registerType As Type) Dim regKey As String =

String.Format("HKEY_CLASSES_ROOT\CLSID $\{\{\{0\}\}\}$ ", registerType.GUID) MxCommands. Unregister(regKey)

End Sub

\#End Region

\#End Region

Private m_application As IApplication

' A creatable com class must have a Public Sub New()

' with no parameters, otherwise, the class will not be

' registered in the com registry and cannot be created

' via Createobject.

Public Sub New()

MyBase. New( )

' TODO: Define values for the public properties

MyBase.m_category = "Io PPR Data Processing" 'localizable text

MyBase.m_caption = "Create Features" 'localizable text

MyBase.m_message = "Create Ellipse Polygons and Center Points

from PPR Data Files" 'localizable text

MyBase.m_toolTip = "Create Ellipse Polygons and Center Points

from PPR Data Files" 'localizable text

MyBase.m_name = "PPR_CreateFeatures" 'unique id, non-

localizable (e.g. "MyCategory_ArcMapcommand")

Try

'TODO: change bitmap name if necessary

Dim bitmapResourceName As String = Me.GetType. Name + ".bmp" MyBase.m_bitmap = New Bitmap (Me.GetType(),

bitmapResourceName)

Catch ex As Exception

System.Diagnostics.Trace.WriteLine(ex.Message, "Invalid

Bitmap")

End Try

End Sub

Public Overrides Sub Oncreate(ByVal hook As object)

If Not hook Is Nothing Then

m_application = CType (hook, IApplication $)$

'Disable if it is not ArcMap

If Typeof hook Is IMxApplication Then

MyBase.m_enabled = True

Else

End If

MyBase.m_enabled $=$ False

End If 


\author{
' TODO: Add other initialization code \\ End Sub \\ Public Overrides Sub OnClick() \\ Dim formParser As New frmParser \\ formParser. ShowDialog( ) \\ End Sub \\ End Class
}




\section{B.3 Code for Create Features ArcCatalog Tool Button}

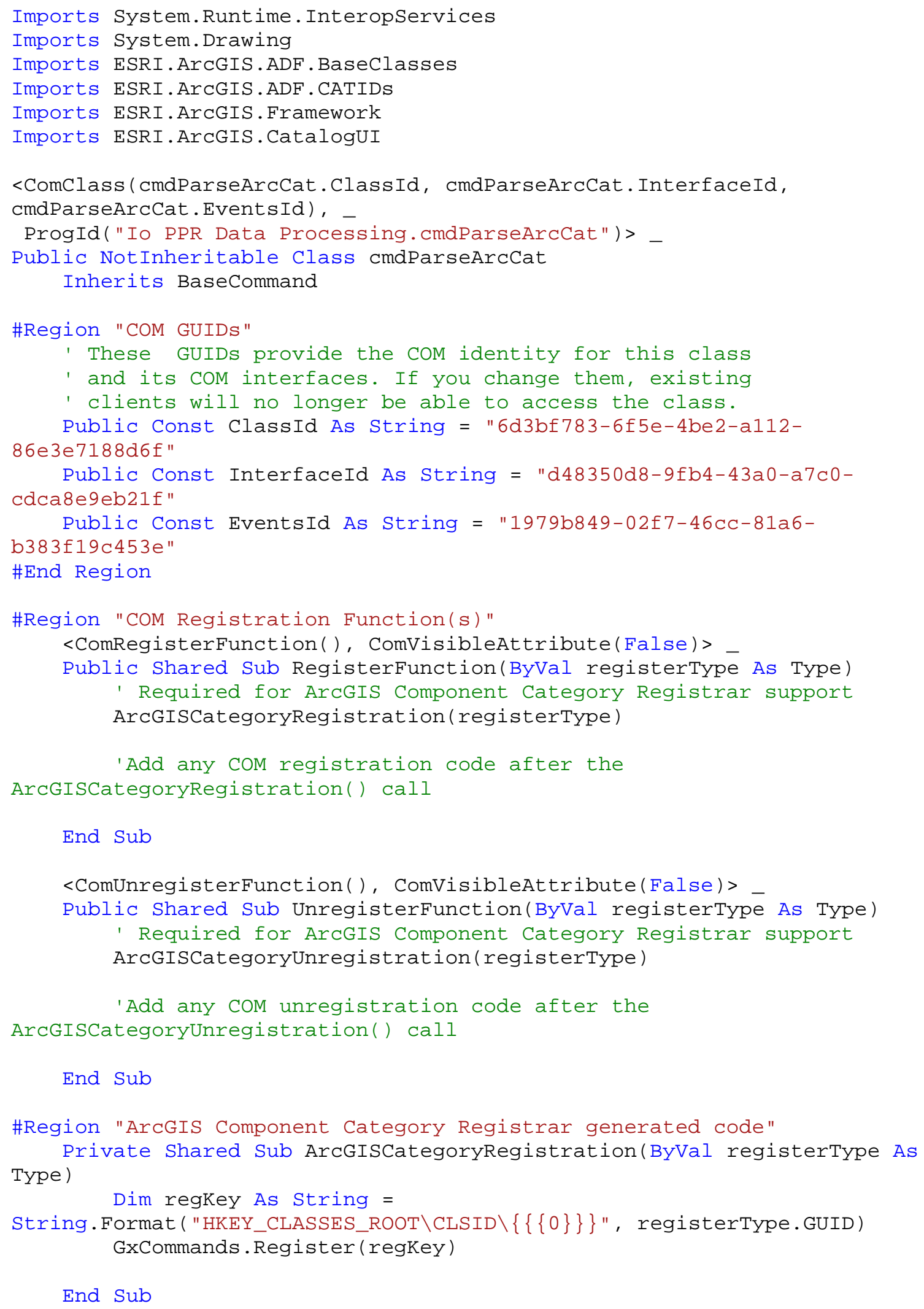


Private Shared Sub ArcGISCategoryUnregistration(ByVal registerType As Type)

Dim regKey As String =

String.Format ("HKEY_CLASSES_ROOT\CLSID $\{\{\{0\}\}\}$ ", registerType.GUID)

GxCommands. Unregister(regKey)

End Sub

\#End Region

\#End Region

Private m_application As IApplication

' A creatable com class must have a Public Sub New()

' with no parameters, otherwise, the class will not be

' registered in the com registry and cannot be created

' via Createobject.

Public Sub New()

MyBase. New( )

' TODO: Define values for the public properties

MyBase.m_category = "Io PPR Data Processing" 'localizable text

MyBase.m_caption = "Create Features" 'localizable text

MyBase.m_message = "Create Ellipse Polygons and Center Points

from PPR Data Files" 'localizable text

MyBase.m_toolTip = "Create Ellipse Polygons and Center Points

from PPR Data Files" 'localizable text

MyBase.m_name = "PPR_CreateFeatures" 'unique id, non-

localizable (e.g. "MyCategory_ArcMapCommand")

Try

'TODO: change bitmap name if necessary

". bmp"

Dim bitmapResourceName As String = Me.GetType (). Name +

bitmapResourceName)

Catch ex As Exception

Bitmap")

System.Diagnostics.Trace.WriteLine(ex.Message, "Invalid

End Try

End Sub

Public Overrides Sub Oncreate(ByVal hook As object)

If Not hook Is Nothing Then

m_application $=$ CType (hook, IApplication $)$

'Disable if it is not ArcCatalog

If Typeof hook Is IGXApplication Then

Else MyBase.m_enabled = True

End If

MyBase.m_enabled $=$ False

End If 


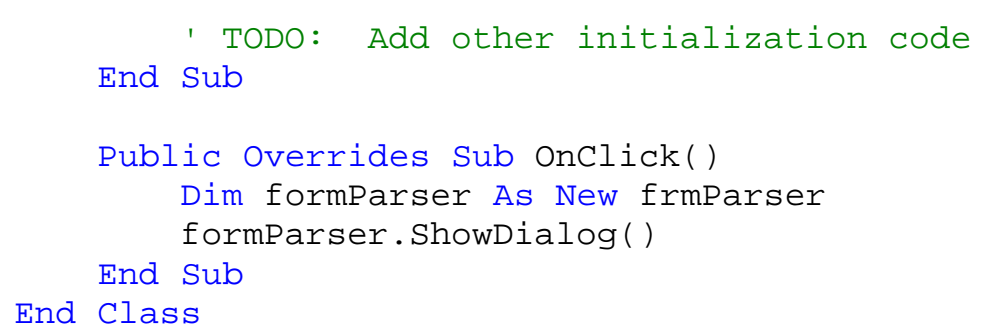




\section{B.4 Code for Set Temperature Symbology Dialog Box}

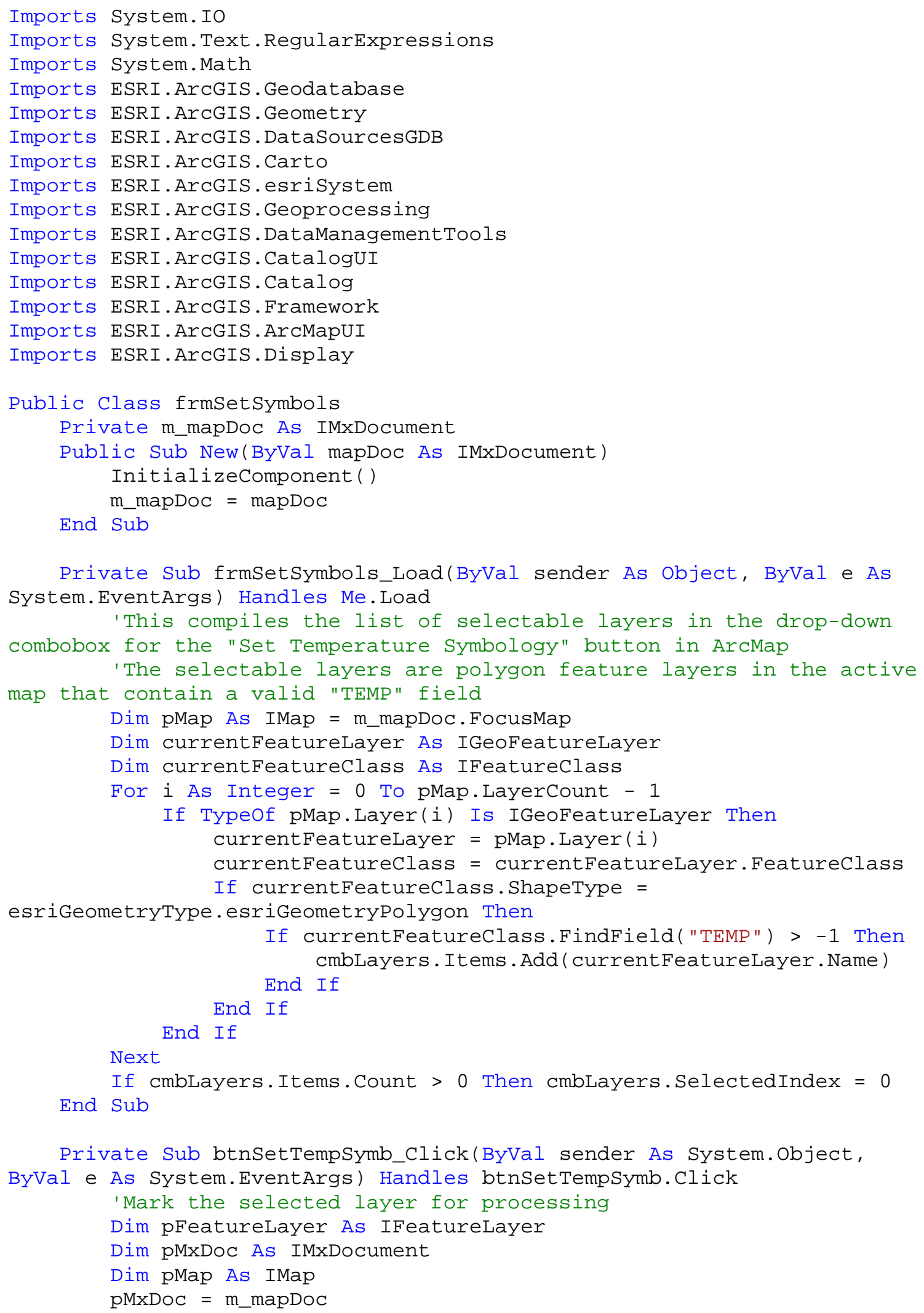

Private Sub btnSetTempsymb_Click(ByVal sender As System.object, Byval e As System.EventArgs) Handles btnSetTempSymb.Click 


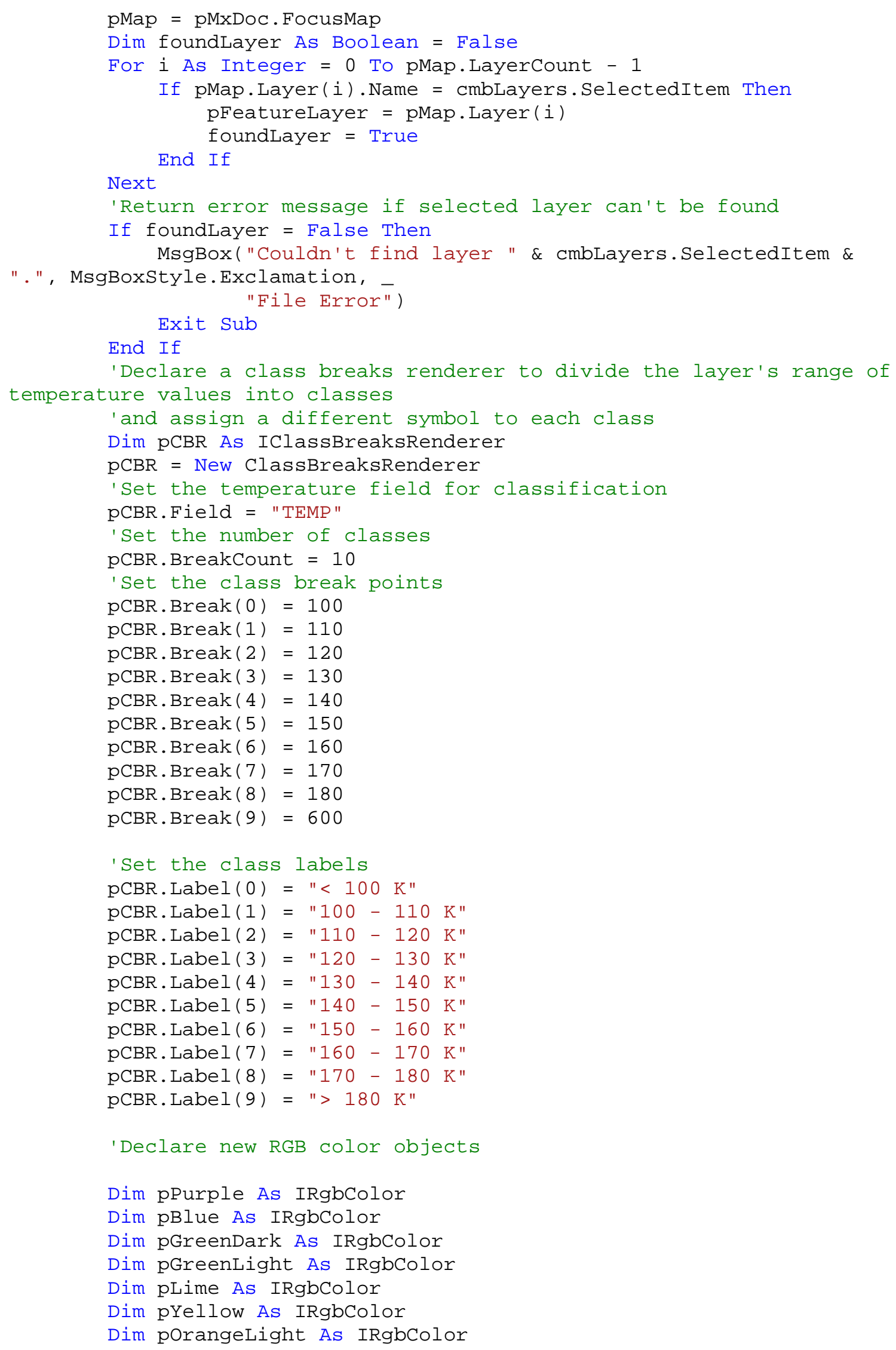




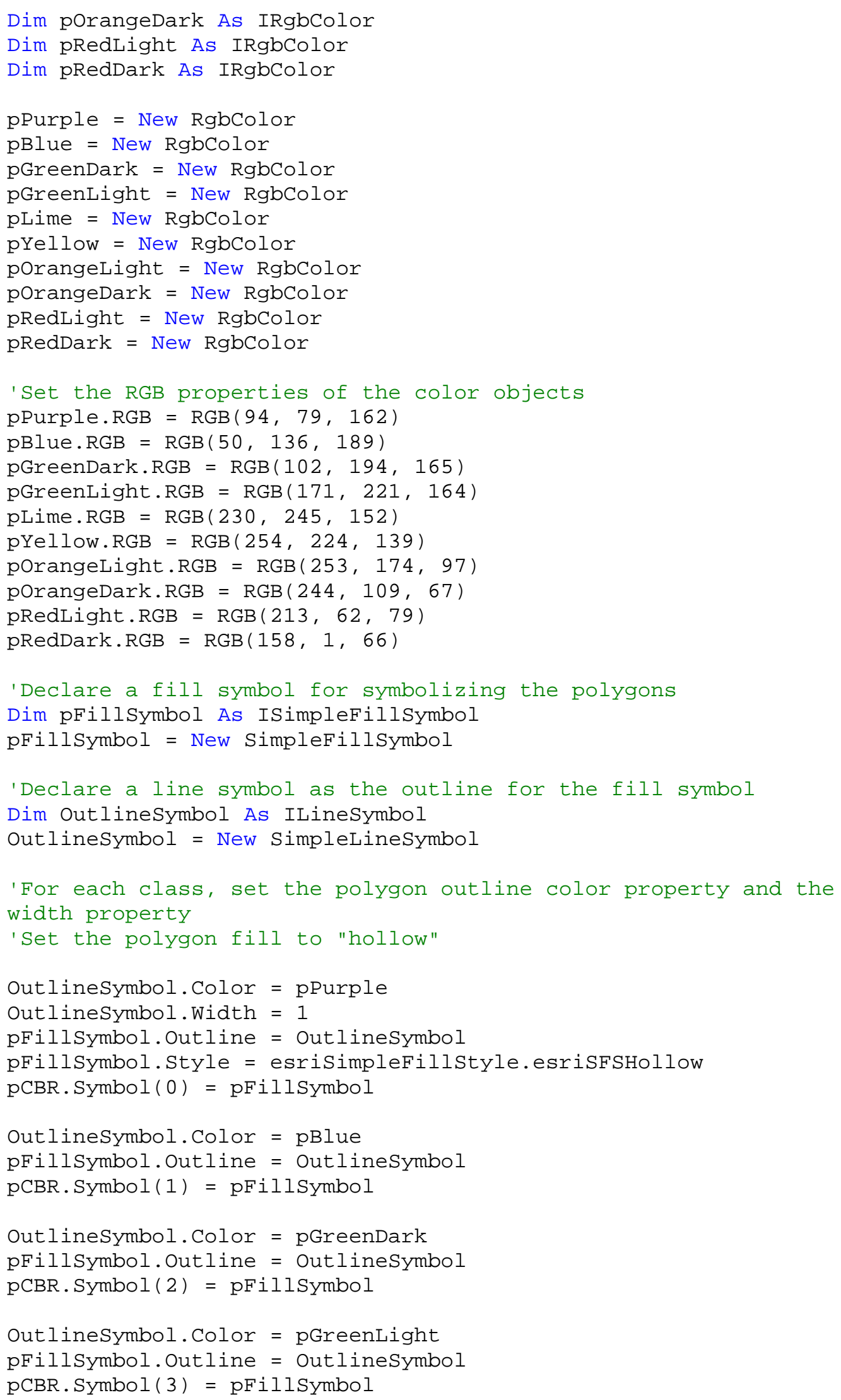




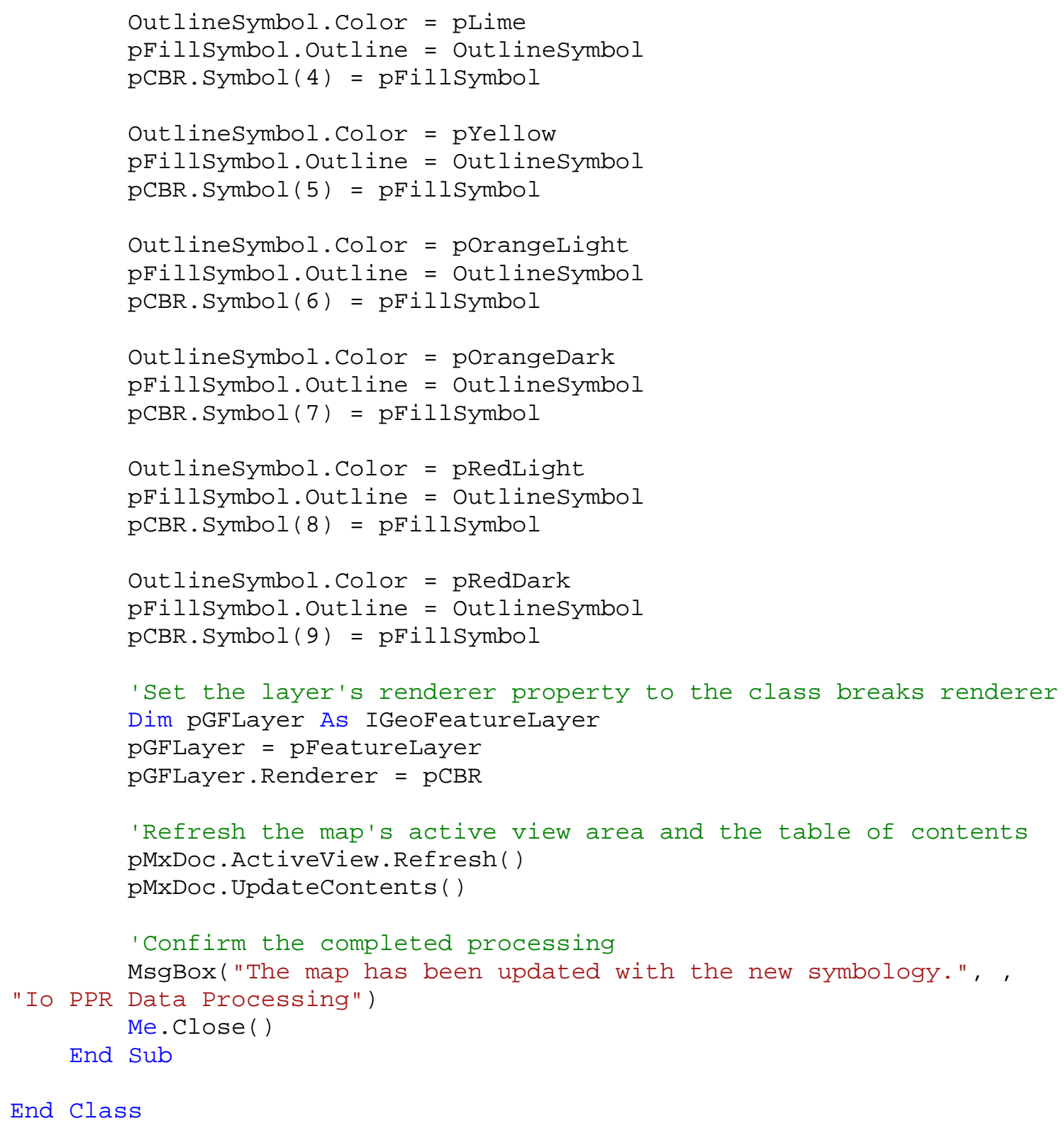




\section{B.5 Code for Set Temperature Symbology ArcMap Tool Button}

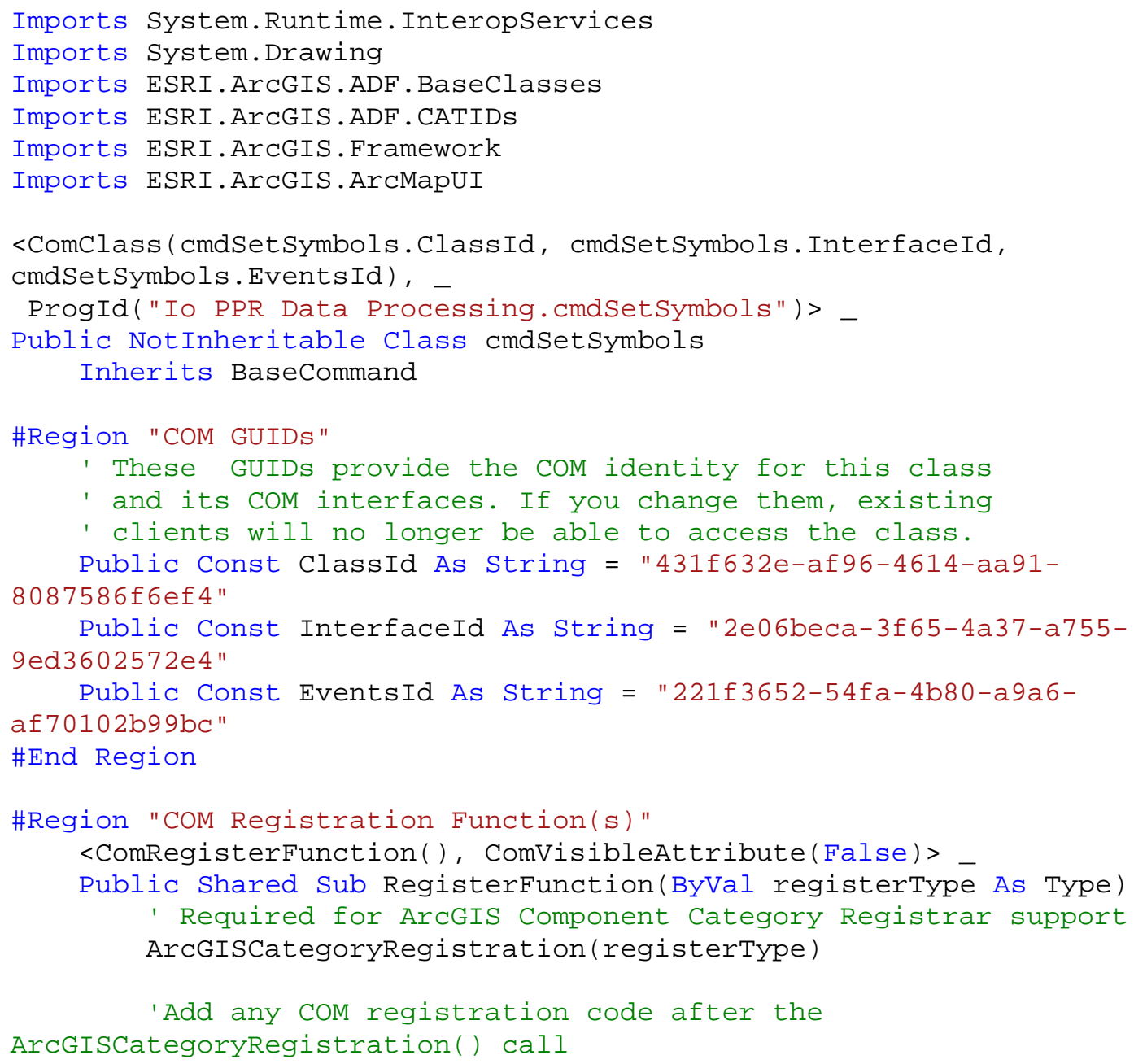

End Sub

$<$ ComUnregisterFunction( ), ComVisibleAttribute(False) >

Public Shared Sub UnregisterFunction(ByVal registerType As Type)

' Required for ArcGIS Component Category Registrar support ArcGISCategoryUnregistration(registerType)

'Add any COM unregistration code after the ArcGISCategoryUnregistration() call

End Sub

\#Region "ArcGIS Component Category Registrar generated code"

Private Shared Sub ArcGISCategoryRegistration(ByVal registerType As Type )

Dim regkey As String =

String.Format("HKEY_CLASSES_ROOT\CLSID $\{\{\{0\}\}\} "$, registerType.GUID)

MxCommands.Register (regKey)

End Sub 
Private Shared Sub ArcGISCategoryUnregistration(ByVal registerType As Type) Dim regKey As String = String.Format("HKEY_CLASSES_ROOT\CLSID $\{\{\{0\}\}\}$ ", registerType.GUID) MxCommands. Unregister(regKey)

End Sub

\#End Region

\#End Region

Private m_application As IApplication

Private m_mxDoc As IMxDocument

' A creatable com class must have a Public Sub New()

' with no parameters, otherwise, the class will not be

' registered in the COM registry and cannot be created

' via Createobject.

Public Sub New()

MyBase. New( )

' TODO: Define values for the public properties

MyBase.m_category = "Io PPR Data Processing" 'localizable text

text

MyBase.m_caption = "Set Temperature Symbology" 'localizable

MyBase.m_message = "Set Ellipse Polygon Outline Color to

correspond with Regional Temperature" 'localizable text

MyBase.m_toolTip = "Set Ellipse Polygon Outline Color to

correspond with Regional Temperature" 'localizable text

MyBase.m_name = "PPR_SetSymbols" 'unique id, non-localizable

(e.g. "MyCategory_ArcMapCommand")

Try

'TODO: change bitmap name if necessary

". bmp"

Dim bitmapResourceName As String = Me.GetType(). Name +

bitmapResourceName)

Catch ex As Exception

Bitmap")

System.Diagnostics.Trace.WriteLine(ex.Message, "Invalid

End Try

End Sub

Public Overrides Sub oncreate(ByVal hook As object)

If Not hook Is Nothing Then

m_application = CType (hook, IApplication)

m_mxDoc $=$ m_application. Document

'Disable if it is not ArcMap

If Typeof hook Is IMxApplication Then

Else

MyBase.m_enabled = True

MyBase.m_enabled $=$ False 


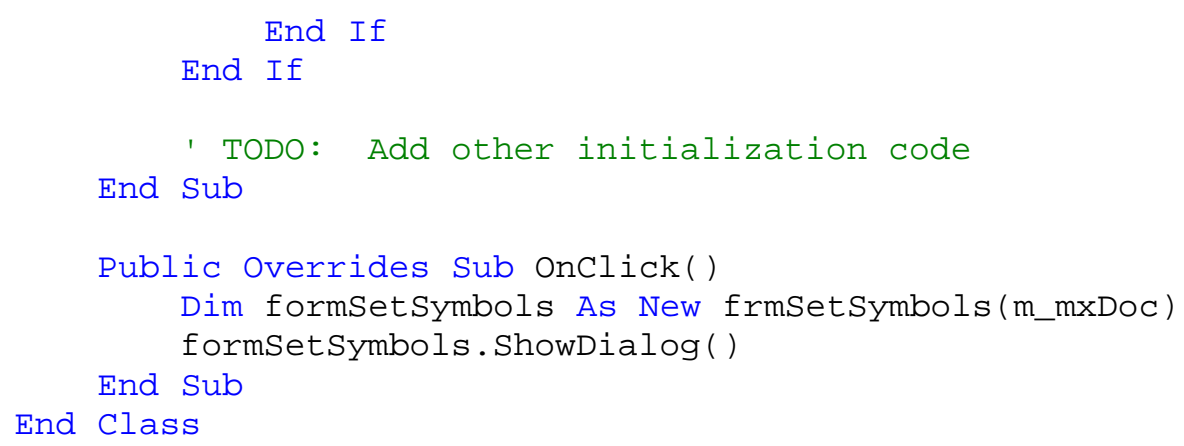




\section{B.6 Code for Io PPR Data Processing Toolbar}

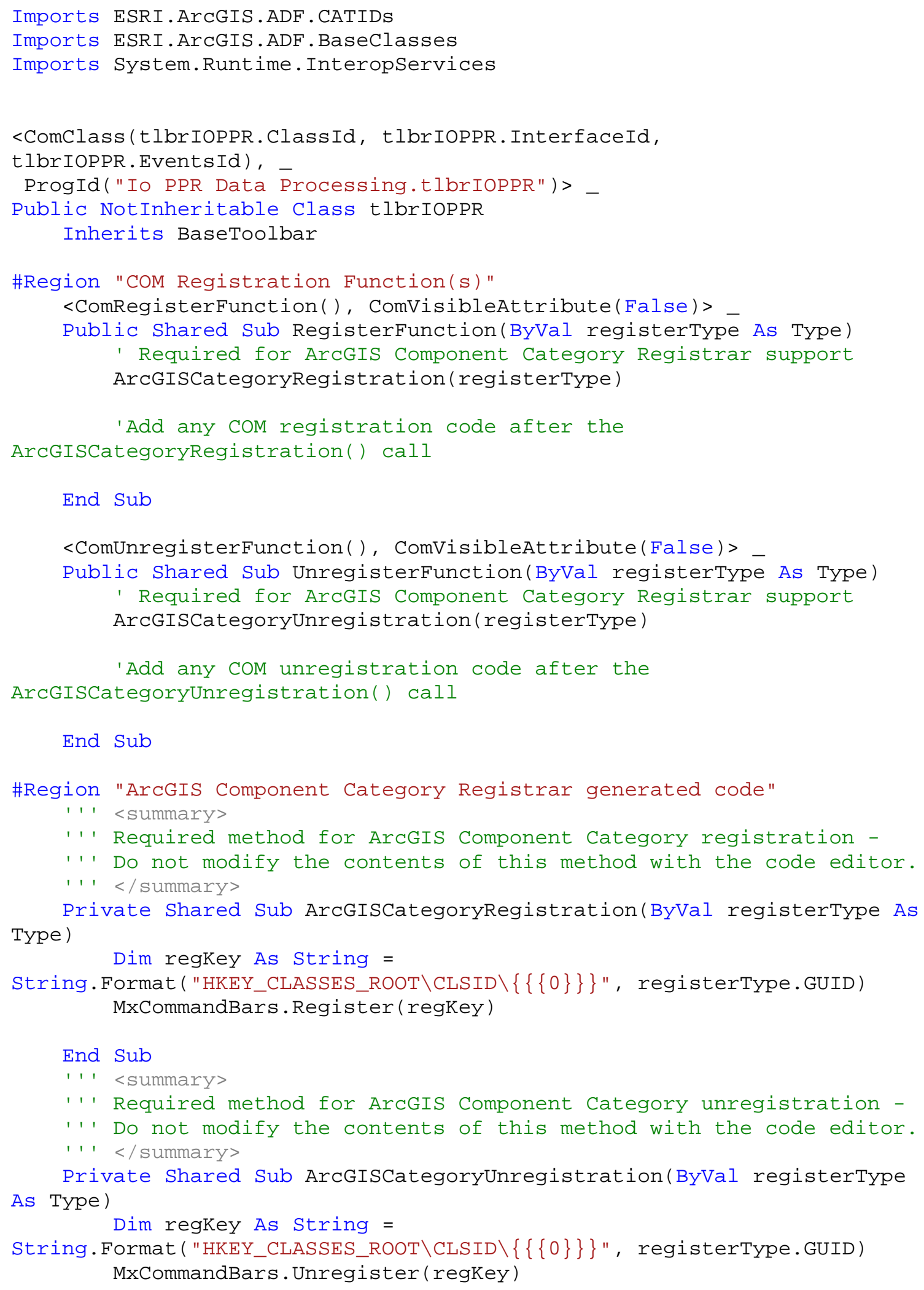


End Sub

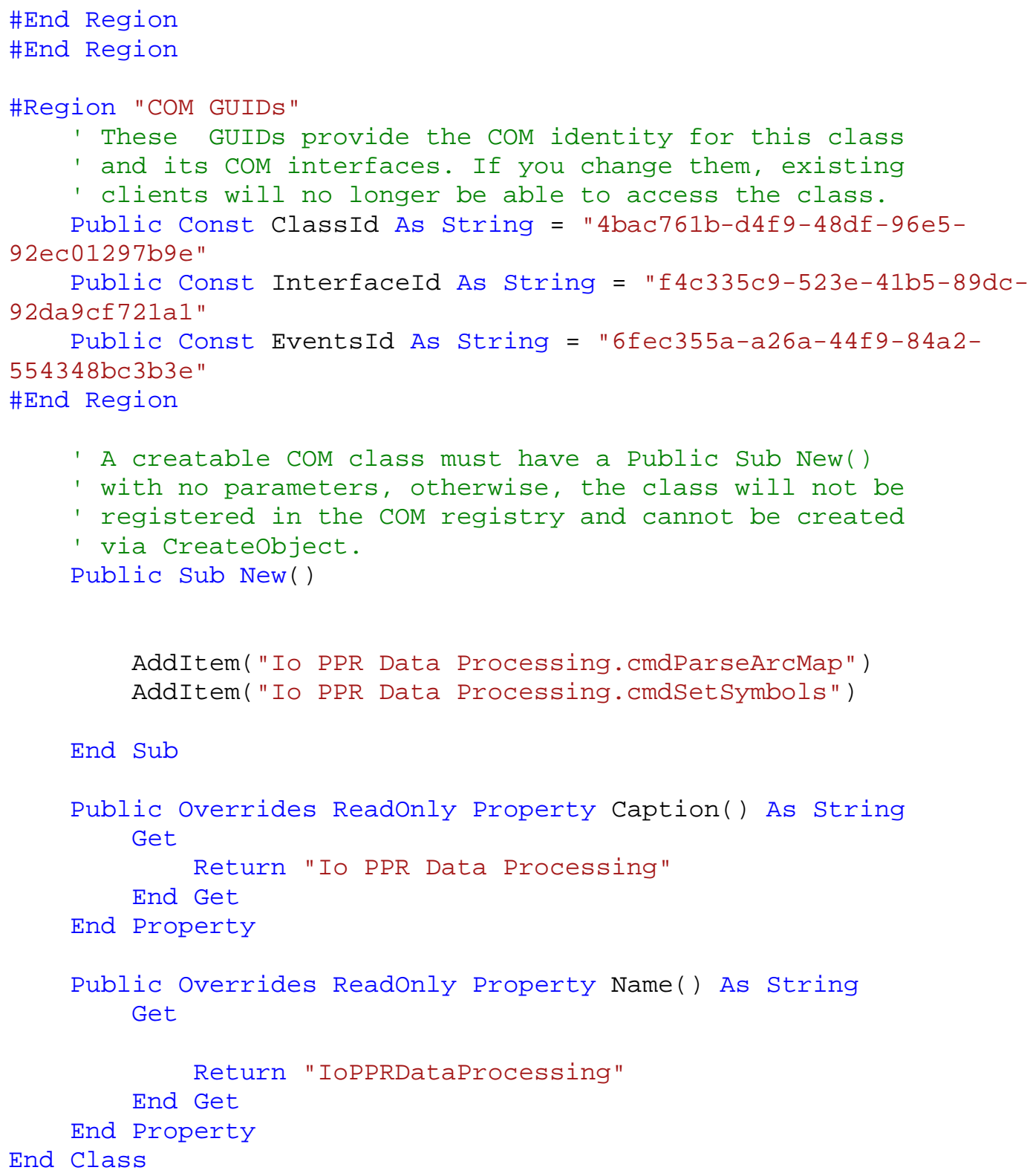

Public Const InterfaceId As String = "f4c335c9-523e-41b5-89dc92da9cf721a1"

Public Const EventsId As String = "6fec355a-a26a-44f9-84a2$554348 b c 3 b 3 e "$

End Sub

Public Overrides Readonly Property Caption() As String Get End Get 



\title{
Appendix C. "Create a command by inheriting from BaseCommand" Documentation from the ESRI Resource Center
}

Create a command by inheriting from BaseCommand

\author{
Create a command by inheriting from BaseCommand
}

This document was published with ArcGIS 9.3. A 2.2 version also exists.

Summary

This walkthrough shows how to create a custom command by inheriting from the ESRI BaseCommand class. Inheriting the BaseCommand allows you to create commands easier than directly implementing the ICommand interface in ESRI.ArcGIS.SystemUI. This command zooms the extent of the selected layer.

Click here to get the sample associated with this walkthrough.

\begin{tabular}{|l|l|}
\hline Development licensing & Deployment licensing \\
\hline ArcView & ArcView \\
\hline ArcEditor & ArcEditor \\
\hline ArcInfo & ArcInfo \\
\hline
\end{tabular}

In this topic

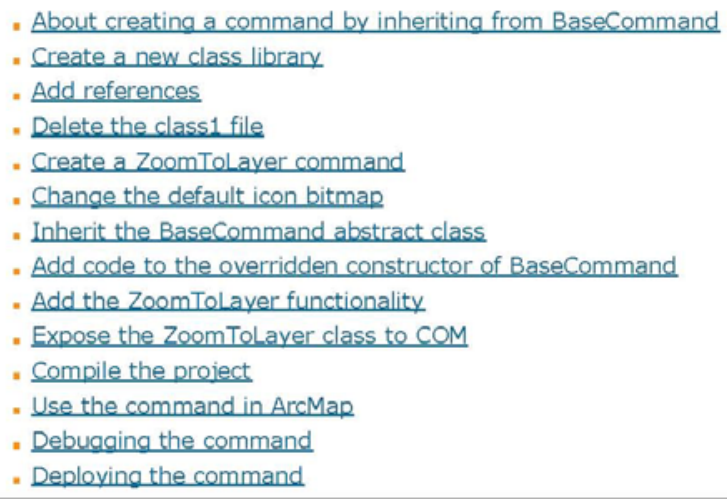

\section{About creating a command by inheriting from BaseCommand}

This walkthrough shows how to create a simple command that zooms the extent of the selected layer. This command is written by inheriting from the ESRI BaseCommand class available in the Application Developer Framework (ADF) assembly and includes the following steps.

\section{Create a new class library}

1. Open Visual Studio 2005.

While this walkthrough refers to and shows screen shots from Visual Studio 2005 running on Windows XP, you can also follow it in Visual Studio 2008 or on Windows Vista. The sample associated with this walkthrough contains the solution and project files for Visual Studio 2005 and Visual Studio 2008.

2. Click File, click New, and click Project.

To complete this scenario, use C\# or VB .NET. The code for both is shown and the full solutions for both languages are included in the associated sample. However, this topic only shows the C\# dialog boxes in the screen shots, since the dialog boxes and views you interact with in VB. NET are very similar.

3. In the New Project dialog box click the Visual C\# or Visual Basic Projects node under the Project types pane, click the ArcGIS node, then click the Desktop node.

Depending on whether you selected C\# or VB .NET as your main language when you installed Visual Studio, the other language will be in the Other Languages node under the Project types pane.

4. Click the Class Library (ArcMap) template under the Templates pane. 
5. Name the project CommandInheritingBaseCommand and browse to the location where you want to save the project. See the following screen shot:

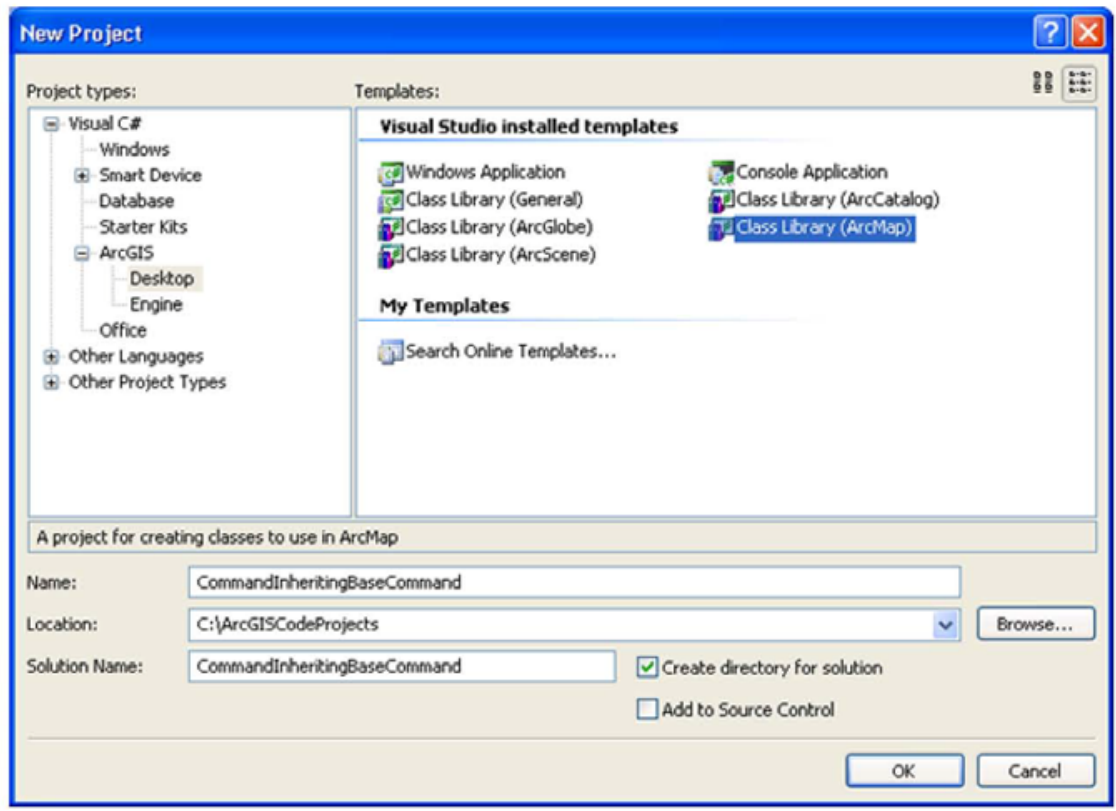

In the associated sample, the solution and project files have 2005 or 2008 appended on the end of the name to indicate which version of Visual Studio (either Visual Studio 2005 or Visual Studio 2008) uses them.

6. Click OK on the New Project dialog box to create the new project.

If the Create directory for solution check box is selected, Visual Studio creates a subdirectory of the selected directory to store your project files. The name of the new directory will be the project name you specified.

\section{Add references}

You will need to add references to the ESRI object libraries to your C\# project and reference System.Drawing.

In VB 6 or VC++ ArcObjects projects, add a reference to the ESRI object libraries, which contains information about Component Object Model (COM) objects. Although .NET cannot use COM objects directly, the COM interoperability services provided by the .NET framework allows you to use COM objects in your. NET project. Using the tools available in Visual Studio 2005, you can create a runtime callable wrapper, which allows a .NET component to create and call methods on a COM object. However, ESRI provides. NET runtime callable wrappers for you and you will use them to instantiate and make calls on the objects in the ESRI object libraries from your. NET project.

ESRI also provides the ESRI.ArcGIS.ADF assembly that will be used in this walkthrough. It contains

the ESRI.ArcGIS.ADF.BaseClasses namespace, which provides abstract classes you inherit from to help create a command or tool. It also contains the ESRI.ArcGIS.ADF.CATIDs namespace that contains classes to help you register your components to ESRI component categories.

1. As part of the new ArcGIS Visual Studio Integration Framework features, the ArcGIS Project Wizard walks you through adding in references to. NET assemblies. The ArcGIS Project Wizard appears after you click OK from the New Project dialog box shown previously. Although there are other formats for viewing the available ESRI references, use the default Filter by: Product Features view. See the following screen shot: 
Create a command by irheriting from BaseCommand

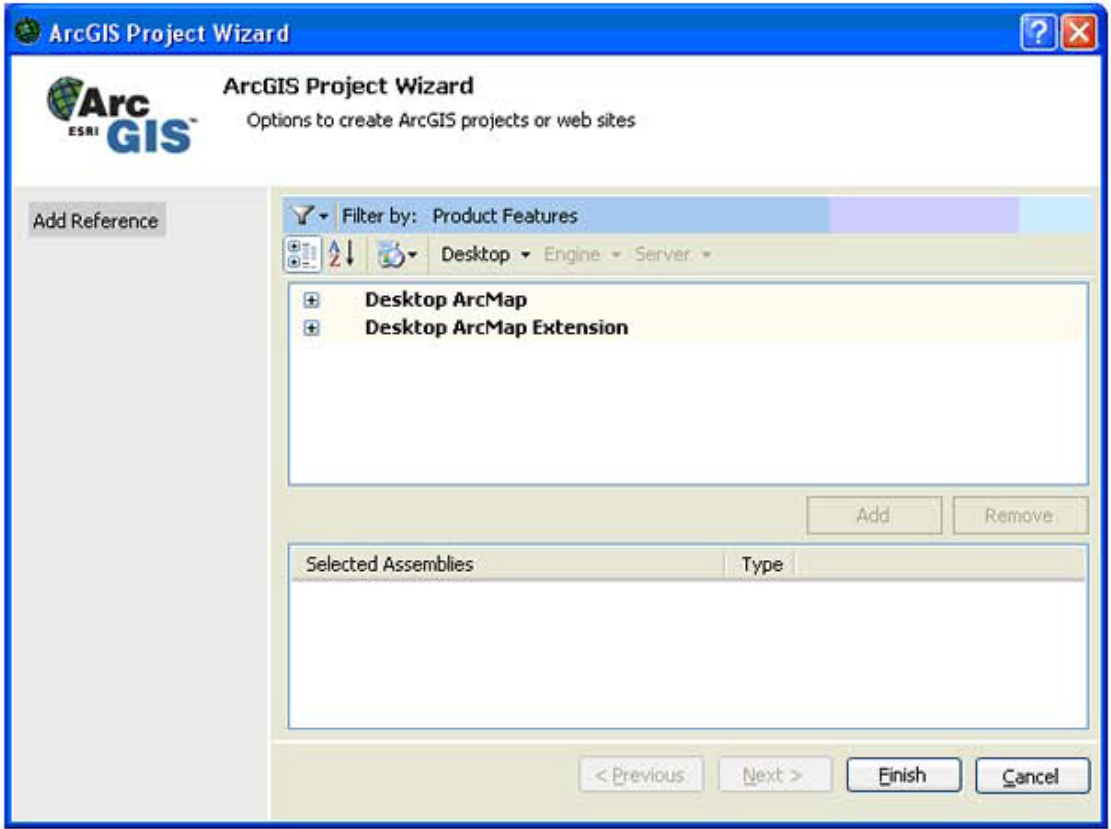

2. Since you are creating an ArcGIS Desktop application, only assemblies relevant for ArcGIS Desktop display. Click the Desktop ArcMap node and select the following assemblies: ESRI .ArcG IS.ADF, ESRI.ArcG IS.ArcMapUI, ESRI.ArcGIS.Carto, ESRI .ArcGIS.Framework, ESRI.ArcGIS.Geometry, ESRI.ArcG IS.System, and ESRI .ArcGIS.SystemUI. To select multiple items together, press and hold the Ctrl key and click each item you want to select. Click Add to have your references appear in the Selected Assemblies pane. See the following screen shot: 
Create a command by irheriting from BaseCommand

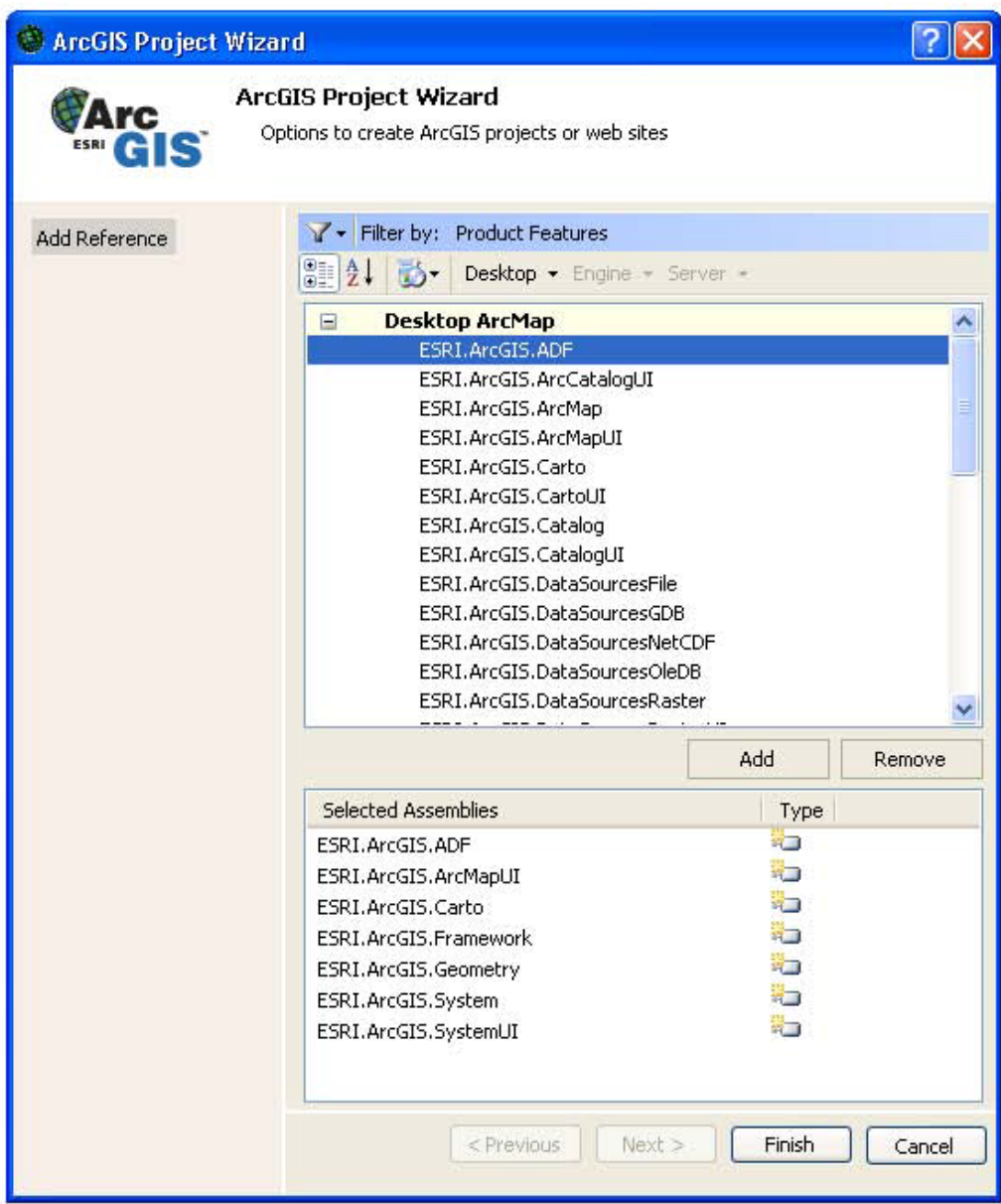

3. Click Finish to create your project.

4. You still need a reference to System.Drawing; therefore, click the Project menu, click Add Reference, click the .NET tab, then click the System. Drawing assembly under the Component Name area. See the following screen shot: 
Create a command by inheriting from BaseCommand

\begin{tabular}{|c|c|c|c|c|c|c|c|}
\hline \multicolumn{5}{|c|}{ Add Reference } & \multicolumn{3}{|c|}{$?$} \\
\hline .NET & $\mathrm{COM}$ & Projects & Browse & Recent & & & \\
\hline \multicolumn{5}{|c|}{ Component Name - } & Version & Runtime & $\hat{\imath}$ \\
\hline \multicolumn{5}{|c|}{ System.Configuration } & 2.0 .0 .0 & v2.0.50727 & \\
\hline \multicolumn{5}{|c|}{ System.Configuration. Install } & 2.0 .0 .0 & $\vee 2.0 .50727$ & \\
\hline \multicolumn{5}{|c|}{ System.Data } & 2.0 .0 .0 & $\mathrm{v} 2.0 .50727$ & \\
\hline \multicolumn{5}{|c|}{ System.Data.OracleClient } & 2.0 .0 .0 & v2.0.50727 & \\
\hline \multicolumn{5}{|c|}{ System.Data.SqlClient } & 3.0 .3600 .0 & $\mathrm{v} 2.0 .50727$ & \\
\hline \multicolumn{5}{|c|}{ System.Data.SqlXml } & 2.0 .0 .0 & $\mathrm{v} 2.0 .50727$ & \\
\hline \multicolumn{5}{|c|}{ System.Deployment } & 2.0 .0 .0 & $\mathrm{v} 2.0 .50727$ & \\
\hline \multicolumn{5}{|c|}{ System.Design } & 2.0 .0 .0 & $\mathrm{v} 2.0 .50727$ & \\
\hline \multicolumn{5}{|c|}{ System.DirectoryServices } & 2.0 .0 .0 & $\mathrm{v} 2.0 .50727$ & \\
\hline \multicolumn{5}{|c|}{ System.DirectoryServices.Protocols } & 2.0 .0 .0 & $\mathrm{v} 2.0 .50727$ & \\
\hline \multicolumn{5}{|c|}{ System.Drawing } & 2.0 .0 .0 & $\mathrm{v} 2.0 .50727$ & \\
\hline \multicolumn{5}{|c|}{ System.Drawing.Design } & 2.0 .0 .0 & $\mathrm{v} 2.0 .50727$ & \\
\hline \multicolumn{5}{|c|}{ System.EnterpriseServices } & 2.0 .0 .0 & $\mathrm{v} 2.0 .50727$ & \\
\hline \multirow{2}{*}{\multicolumn{5}{|c|}{$\begin{array}{l}\text { System.Management } \\
\text { Svstem.Messaninn }\end{array}$}} & 2.0 .0 .0 & $\mathrm{v} 2.0 .50727$ & \\
\hline & & & & & 2.ก.ก.ก & ท.ก. $5 ก 727$ & \\
\hline \multicolumn{5}{|c|}{$<$ < } & & $>$ & \\
\hline & & & & & OK & Cance & \\
\hline
\end{tabular}

5. Click OK to close the Add Reference dialog box and add the assembly reference.

Recently added references can be seen by clicking the Recent tab in the Add Reference dialog box.

\section{Delete the class1 file}

When your project is created, a class1 file (.cs or .vb depending on the language you chose) is added to your project by default. In this walkthrough, you will not use it. Right-click that file in the Solution Explorer and click Delete. Click OK to permanently delete it.

\section{Create a ZoomToLayer command}

You will use one of the ArcGIS item templates to add a new class for the ZoomToLayerCommand in your project.

1. In the Solution Explorer, right-click the project, click Add, and click New Item.

2. In the Add New Item dialog box, click to expand the project items, then click ArcGIS under the Categories pane. Click Base Command under the Templates pane. Name the class ZoomToLayer.cs (C\#) or ZoomToLayer.vb (VB .NET) and click Add. See the following screen shot: 
Create a command by irheriting from BaseCommand

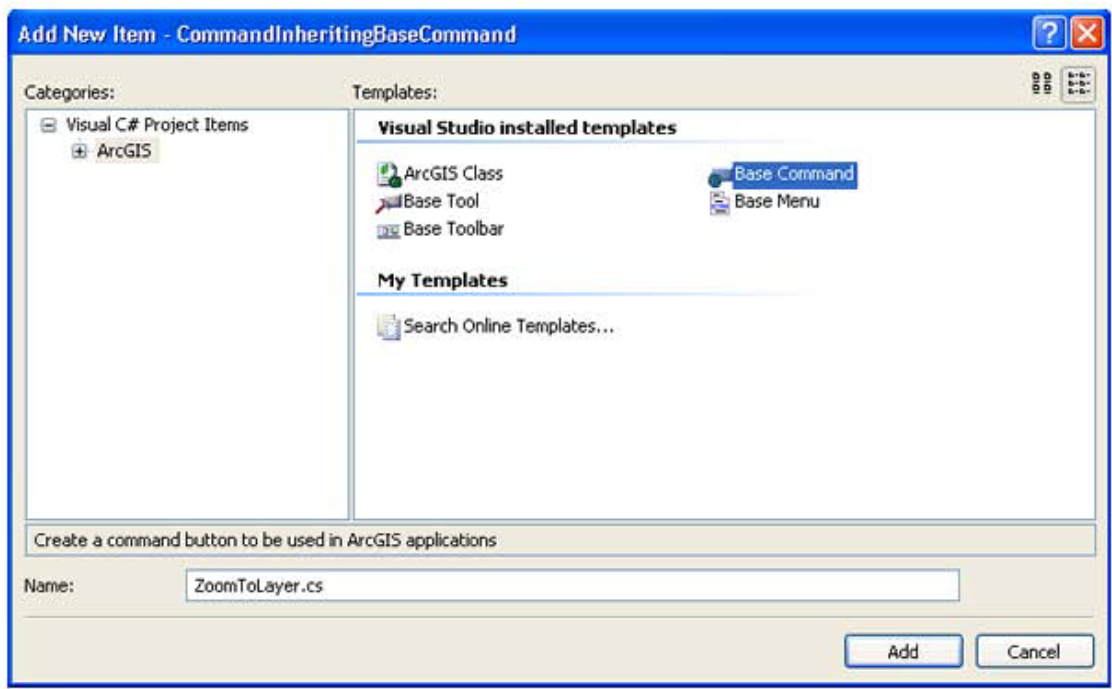

3. When the ArcGIS New Item Wizard Options dialog box appears, click Desktop ArcMap Command and click OK. See the following screen shot:

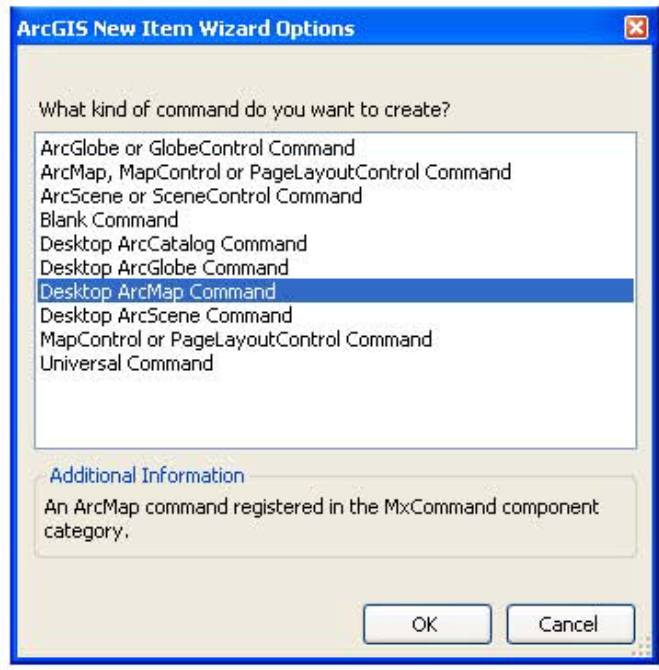

\section{Change the default icon bitmap}

By default, a bitmap is added to your project that will serve as the icon you click in ArcMap to use your custom command You can customize the bitmap used with the image editor. In this example, you will add an existing bitmap file to your project and use this file later to set the icon used for the ZoomToLayer command's button face.

1. In the Solution Explorer, right-click ZoomToLayer.bmp, click Delete, then click OK to permanently delete the Zoom ToLayer.bmp file.

2. In the Solution Explorer, right-click the CommandInheritingB aseCommand project, click Add, and click Existing Item.

3. In the Add Existing Item dialog box, click the Files of type drop-down arrow and select Image Files.

http://resources.esri.com/help/9.3/ArcGISDesktop/dotnet/01001659-cdf8-4579-9c87-2b965e872d84.htm[8/4/2009 2:22:32 PM] 
4. Browse to the Bin Icons directory of your ArcGIS install location, click layer 6.bmp, and click Add to copy it to your project directory. See the following screen shot:

Add Existing Item - CommandlnheritingBaseCommand

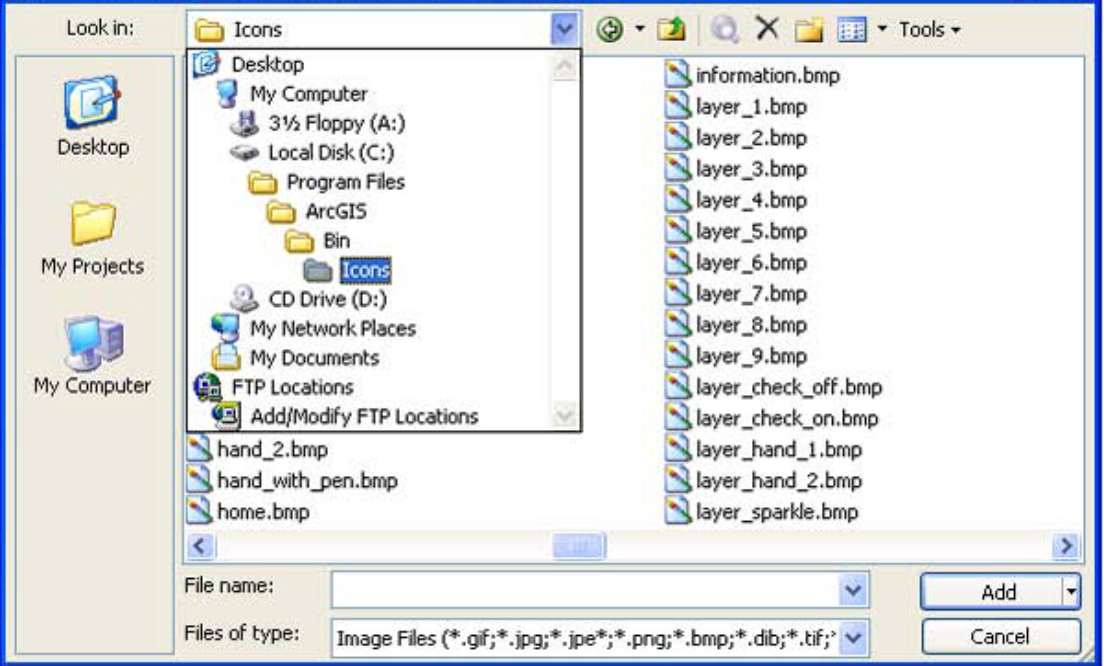

5. Right-click layer 6.bmp in the Solution Explorer and click Properties. In the Properties window, click the Build Action drop-down arrow and select Embedded Resource. Name the bitmap file ZoomToLayer.bmp. See the following screen shot:

\begin{tabular}{|c|c|}
\hline Properties & $-4 x$ \\
\hline ZoomToLayer.bmp & File Properties \\
\hline 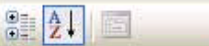 & \\
\hline Build Action & Embedded Resource $\vee$ \\
\hline Copy to Output Dire & Do not copy \\
\hline Custom Tool & \\
\hline Custom Tool Namesf & \\
\hline File Name & ZoomToLayer.bmp \\
\hline Full Path & C:IArcGISCodeProjectsic \\
\hline
\end{tabular}

The bitmap icon file is ready to use later in this walkthrough.

\section{Inherit the Basecommand abstract class}

The next step in writing the custom command is to set your Zoom ToLayer class to inherit from the Basecommand abstract class.

Abstract classes are classes that cannot be instantiated and are frequenty partially implemented or not implemented at all, They are closely related to interfaces; however, they differ significantly from interfaces in that a class can implement multiple interfaces, but it can inherit from only one abstract class.

As part of the new ArcGIS Visual Studio Integration Framework features, when you use the Add Item templates for a Desktop ArcMap Command, most of the plumbing code is done for you. You autom atically get a member variable declared for the IApplication interface, the constructor is set up with stub code, the overridden OnCreate method with the application hook is created, and the overridden Onclick method is stubbed out. See the following code example that shows how the class is set to inherit from $\mathrm{B}$ asecommand: 
$[\mathrm{C} \#]$

public sealed class zoomToLayer: Basecommand

[VB.NET]

Public Notinheritable class zocmTolayer

By convention, member variables are declared at the beginning of the class definition, although the declarations can be placed anywhere within a class. Member variables of a class are often referred to as class fields in .NET.

To use the VB .NET and C\# commands in ArcMap at the same time, the ProgID of the commands has been updated to include a language specifier. See the following code example:

$[\mathrm{C \#}]$

[Progid ( "Command Inhe ritingzasecomenand. zoomTo Layer")

[VB.NET]

<Comclass (ZoomToLayer. Class Id, ZoomtoLayer. InterfaceId, ZoomToLayer. Events Id), -

\section{Add code to the overridden constructor of BaseCommand}

Your class now inherits from BaseCommand. You will start adding code to the plumbing code that has been added for you.

1. Scroll in the code window to find the constructor for the ZoomToLayer class in the ZoomToLayer file. All the properties are empty strings and update their values for the command as shown in the following code example:

[C\#]

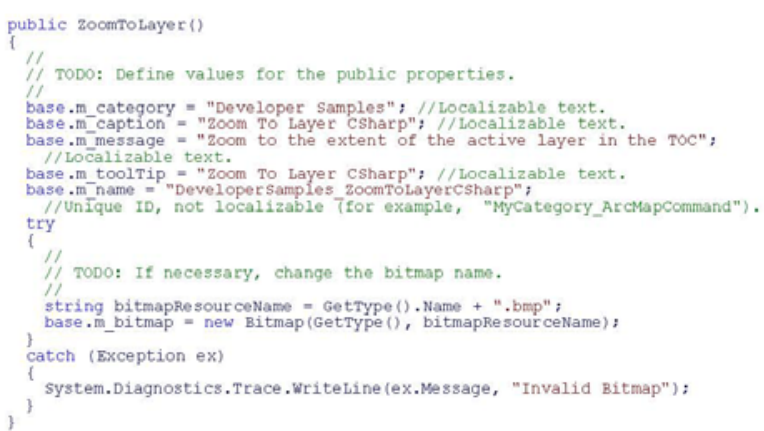

[VB.NET]

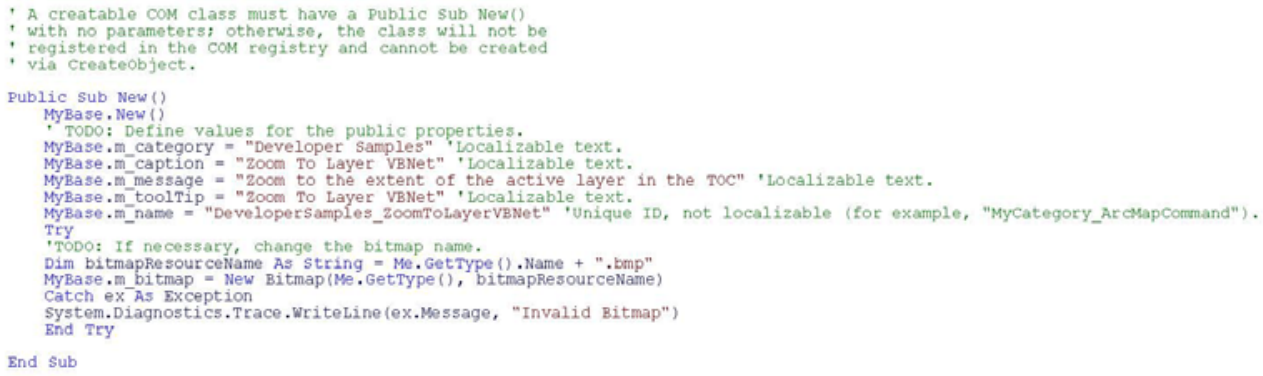

http://resources.esri.com/help/9.3/ArcGISDesktop/dotnet/01c01659-cdf8-4579-9c87-2b965e872d84.htm[8/4/2009 2:22:32 PM] 
2. Since the bitmap is named the same as the class name, the bitmap section placed there by the integrated development environment (IDE) will use the added bitmap.

- The class constructor is a method that is called when the class is created. You can use it to set up members of the class. In VB.NET, the constructor is named New and in C\# its name matches that of the class.

- You can implement the Bitmap, Category, Caption, Name, Message, and Tooltip methods individually; however, it is easier to set the values that should be returned from these methods and rely on the BaseCommand class to provide the implementation for these methods.

- You will leave the other members of ICommand which are not set here to return their default value as implemented by BaseCommand, that is, Enabled $=$ True, Checked $=$ False, HelpFile $="$ "' and HelpContextID $=0$.

\section{Add the ZoomToLayer functionality}

Another new feature of the ArcGIS Visual Studio Integration Framework is ArcGIS snippets. ArcGIS snippets are chunks of ArcObjects reusable code that can be inserted into your project. You can access ArcGIS snippets by right-clicking on the Visual Studio code editor window at the desired insertion point, choosing ArcGIS Snippet Finder from the context menu to open the dialog box, typing in the desired keyword or keywords, clicking the Search button to retrieve the ArcGIS Snippet results, choosing a desired snippet, and clicking the Insert Code button to add the code to the Visual Studio code editor window.

1. In this walkthrough, type zoom in the Keyword(s) text field to narrow your search of ArcGIS snippets, select Zoom to Active Layer in TOC.snippet, and click Insert Code to embed the snippet into your Visual Studio code editor. See the following screen shot: 
Create a command by irheriting from BaseCommand

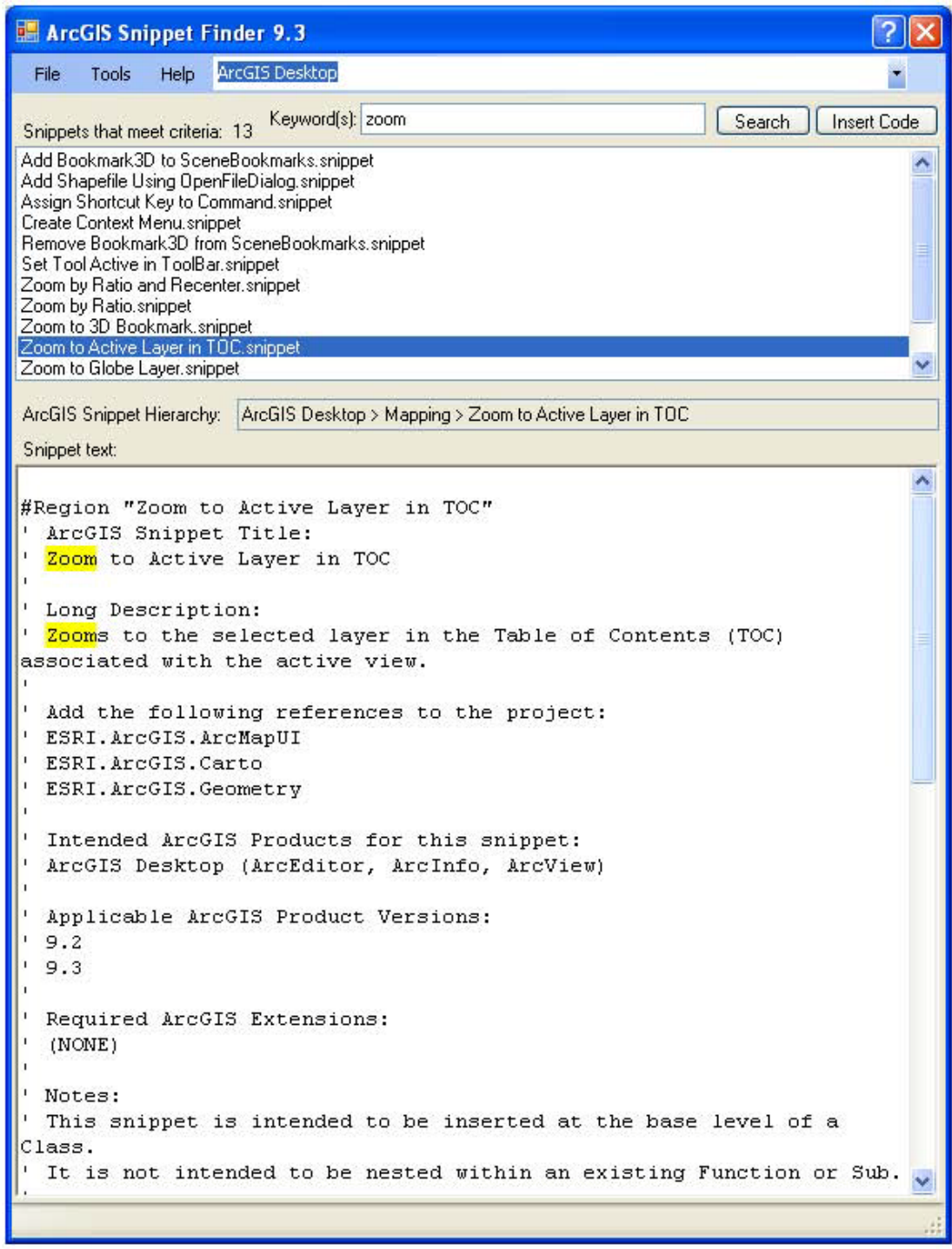

After you click Insert Code, the following code example is added:

[C\#]

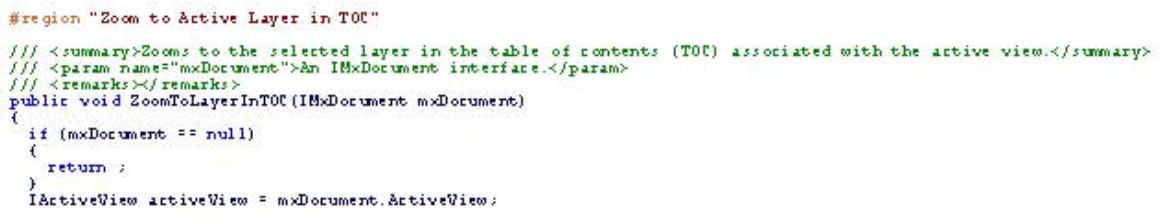

http://resources.esri.com/help/9.3/ArcGISDesktop/dotnet/01001659-cdf8-4579-9c87-2b965e872d84.htm[8/4/2009 2:22:32 PM] 
Create a command by inheriting from BaseCommand

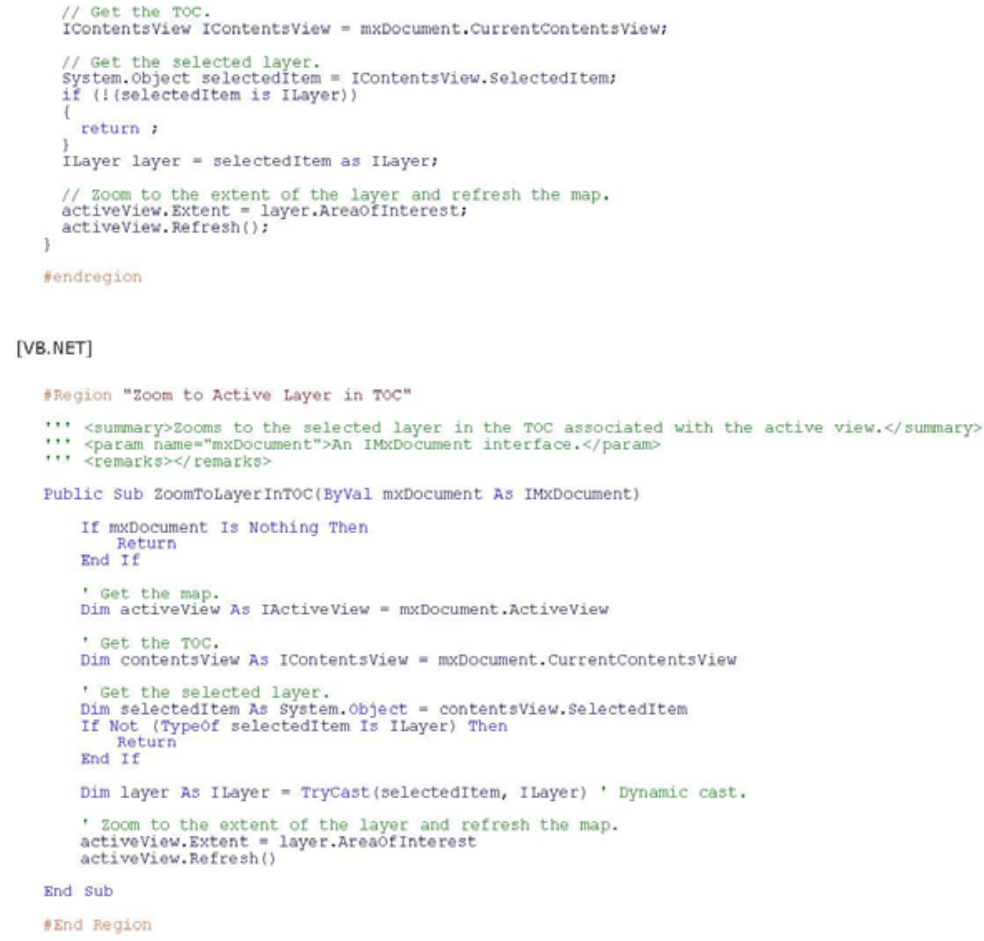

Collapse the ArcGIS snippet by clicking the plus (+) sign next to \# region Zoom to Active Layer in TOC to hide the lines of code in that region (functionality is still available). This makes the file easier to read when you have large amounts of code in it.

2. To take advantage of the functionality for the Zoom to Active Layer in TOC ArcGIS snippet, call the function and supply the appropriate input parameter. The required parameter is an ESRI.ArcGIS.ArcMapUI.IMxDocument interface and you can use another ArcGIS snippet to get the MxDocument to pass in. Open the ArcGIS Snippet Finder dialog box and type MxDocument in the Keyword(s) text field and click Search to obtain the Get MxDocument from ArcMap.snippet. Click Insert Code to embed the snippet into your Visual Studio code editor. See the following screen shot: 
Create a command by irheriting from BaseCommand

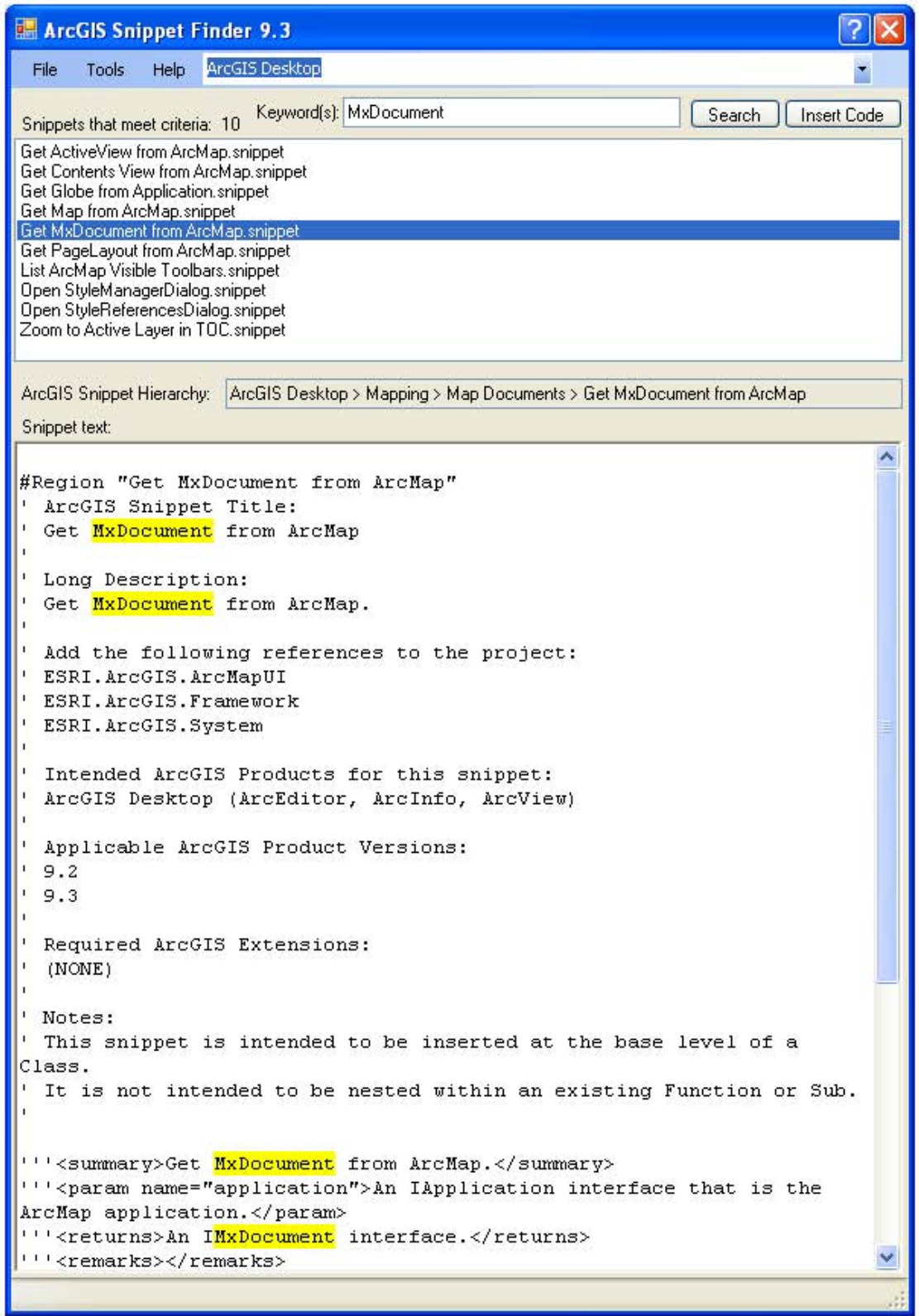

After you click Insert Code, the following code example is added:

[C\#]

\#Fe gion " Get MxDocument from A $A$ reMap"

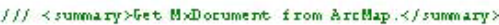

http://resources.esri.com/help/9.3/AroGISDesktop/dotnet/01001659-cdf8-4579-9c87-2b965e872d84.hthn[8/4/2009 2:22:32 PM] 


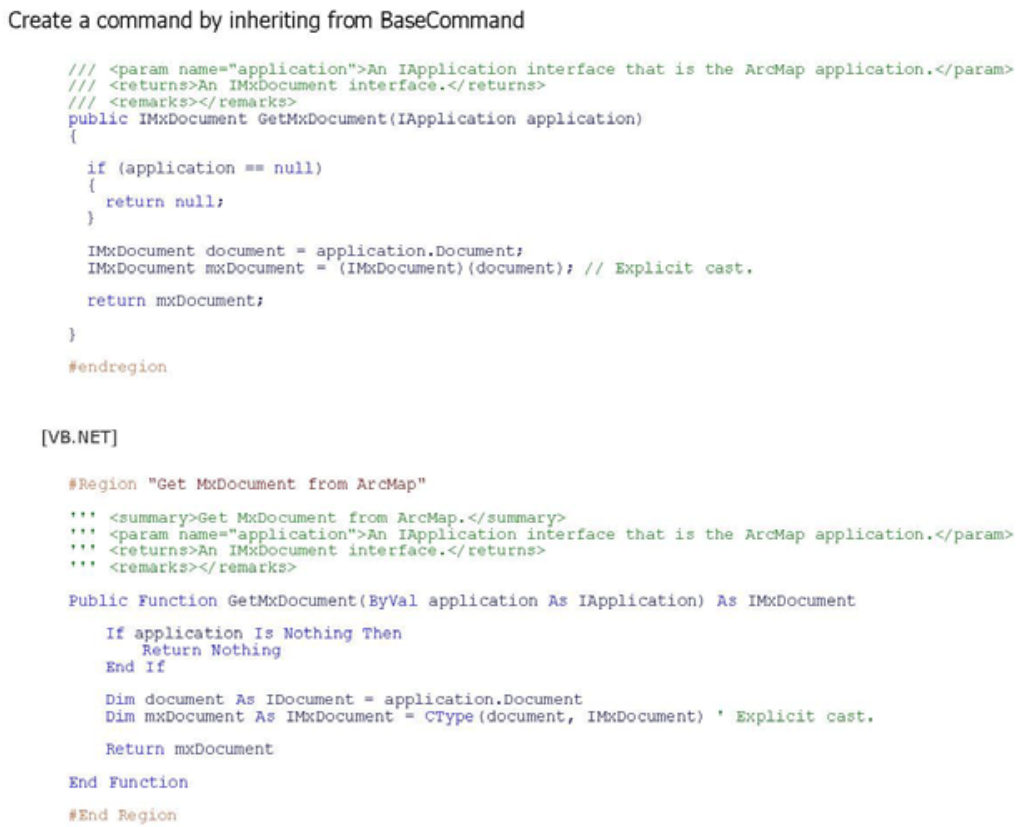

3. Now that the snippets are in place, call them from the OnClick method to execute the code when the custom command is clicked in ArcMap. To call the GetMxDocument method, pass in an

ESRI.ArcGIS.Framework.IApplication interface (previously specified) as member variable m_application. To cal the ZoomToLayerInTOC method, pass in an ESRI.ArcGIS.ArcMapUI.IMxDocument interface that is the return value from the GetMxDocument method. Call both snippets in order as shown in the following code example:

The OnClick function is in the Overriden Class Methods region. If necessary, click the plus (+) sign next to the region to see the method.

$[\mathrm{C} \#]$

III <summary>

public override void onclick ()

IMxDocument mxDocument - GetMxDocument (m_application);$$
\text { zoomToLayer InToc(mxDocument); }
$$

[VB.NET]

Public overrides sub onclick ()
Dim mxDocument As IMxDocument

im mxDocument As IMXDocument $=$ GetMxDocument (m_application)

End sub

\section{Expose the ZoomToLayer class to COM}

ArcGIS expects your command class to be a COM class; therefore specify that the .NET class you have created is also exposed as a COM class by creating a COM callable wrapper. Fortunately, this was done for you by the ArcGIS item template you previously used to create the ZoomToLayer class in your project.

If you want to view the code for this step, expand the \# region COM Registration Function(s) section of your code.

\section{Compile the project}

Do the following to build your project:

1. Make sure you save your project and the work you have done.

http://resources.esri.com/help/9.3/ArcGISDesktop/dotnet/01c01659-cdf8-4579-9c87-2b965e872d84.htm[8/4/2009 2:22:32 PM] 
2. Click the Build menu and click Build Solution.

You can also press Ctrl+Shift+B or press F1 to build your solution.

3. If your project built correctly, a report states the build succeeded in the Output window at the bottom of the Visual Studio .NET IDE.

You can also check the results of the build operation by looking in the subdirectories of your project. By default, you will build a debug version of your project. The dynamic-link library (DLL) that results from the build operation will be stored in the Bin\Debug subdirectory of your project. This directory also contains debug information (.pdb) and a type library (.tlb) file, produced by the Assembly Registration tool.

The obj subdirectory of the project directory contains temporary files used by the compiler and by Visual Studio.

4. If you successfully followed this walkthrough, your build will succeed and you can close Visual Studio now that your custom command has been created. If your build operation did not succeed, click View and click Task List to view the errors, correct the errors as indicated, then close Visual Studio when you have a successful build.

If you double-click the task, the line of code causing the error will automatically be selected.

\section{Use the command in ArcMap}

Do the following to use the custom ZoomToLayer command in ArcMap:

1. Start ArcMap and open a map document that has data layers.

2. Click the Tools menu and click Customize to open the Customize dialog box. Click the Commands tab, then click Developer Samples under the Categories pane.

- If you do not see your Category or Command, verify Register for COM Interop is checked in your Visual Studio Project Properties under the Build tab and rebuild your project. See the following screen shot:

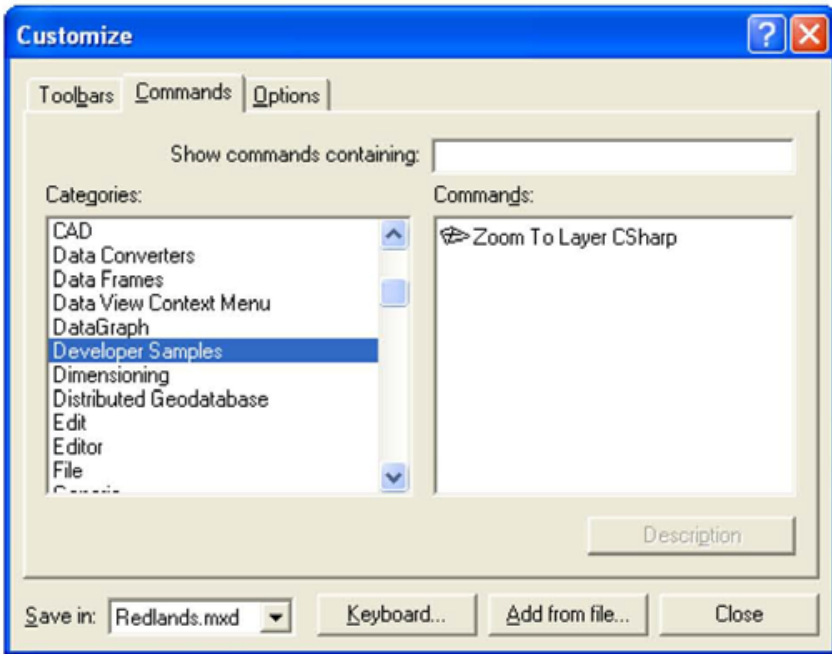

3. In the Commands pane, click the Zoom To Layer CSharp command, and drag it on to an ArcMap toolbar, then click Close on the Customize dialog box. See the following screen shot: 
Create a command by irheriting from BaseCommand

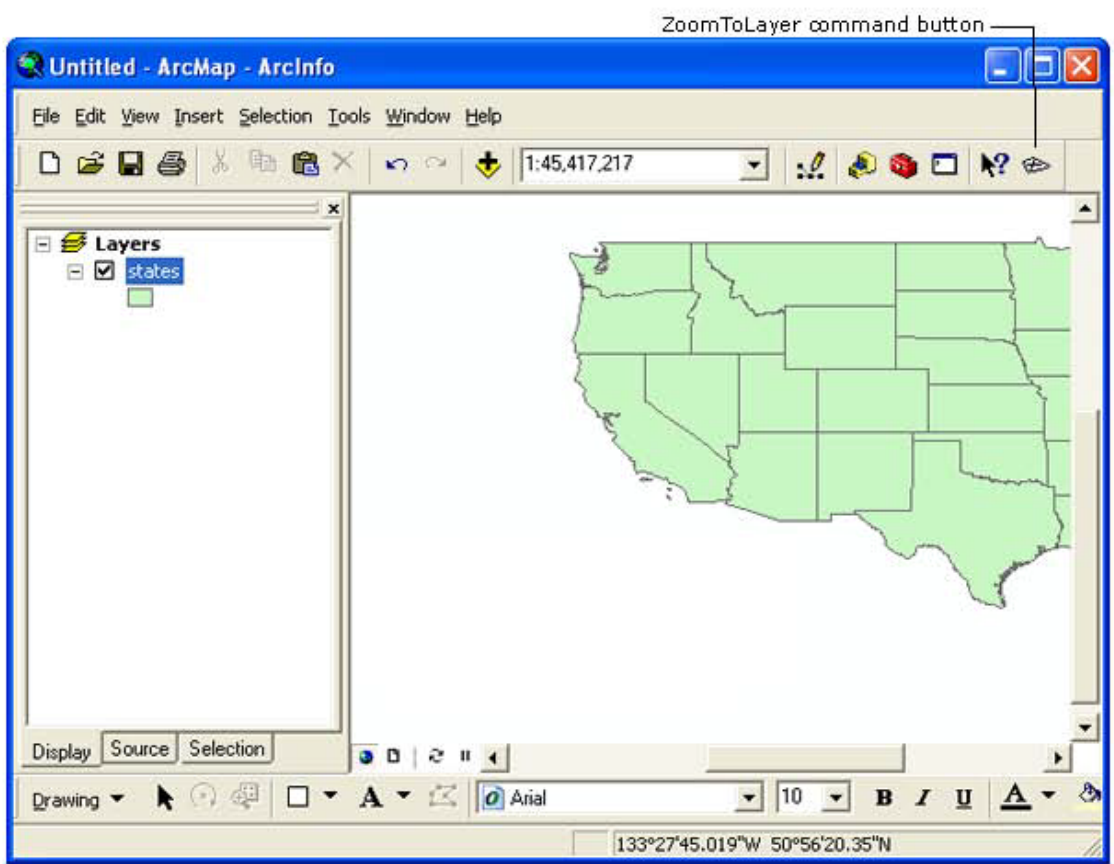

4. Click any layer in the TOC and click the ZoomToLayer button; the map zooms to the extent of that layer. See the following screen shot:

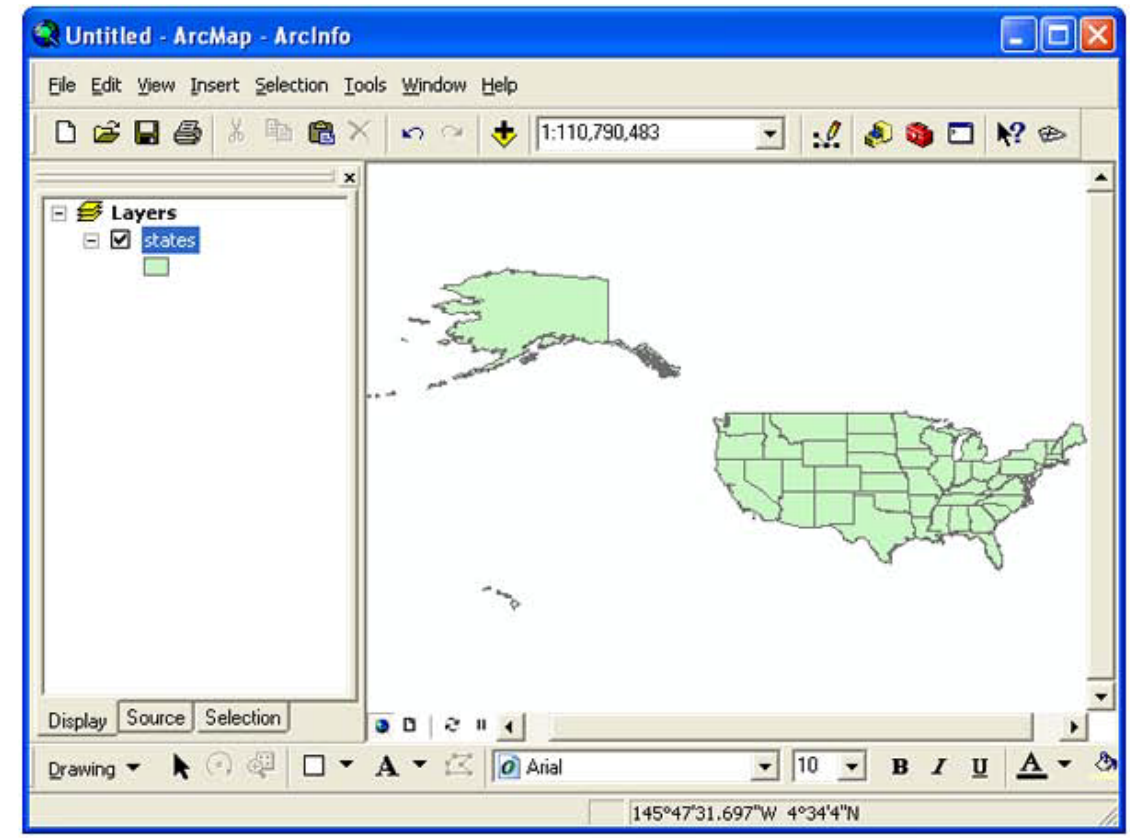

http://resources.esri.com/help/9.3/ArcGISDesktop/dotnet/01001659-cdf8-4579-9c87-2b965e872d84.hton[8/4/2009 2:22:32 PM] 


\section{Debugging the command}

Running the command in debug mode allows you to step through the code when it is executed. This is helpful when you come across bugs in custom commands. While you shouldn't need to debug the command you have created with this walkthrough, it is important to be familiar with these steps when writing your commands.

When you created your project, ArcMap.exe was set as the application to use for debugging by the ArcGIS Visual Studio Integration Framework. The following steps take you through the process of setting it. Set it when you work with a project not created by the ArcGIS Visual Studio Integration Framework or if the project user file (*..csproj.user or ${ }^{*}$.vbproj.user) gets removed (as this is where the debugging setting is stored).

1. Return to the solution in the Visual Studio 2005 IDE.

2. Verify that ArcMap.exe is set as the external program to use for debugging. This is another feature set for you by the ArcGIS project template when you create your project.

a. Right-click the CommandInheritingBaseCommand project in the Solution Explorer window and click Properties.

b. Click the Debug tab on the property page.

c. Under Start Action, set Start external program to use ArcMap.exe in the Bin folder of your ArcGIS install directory. See the following screen shot:

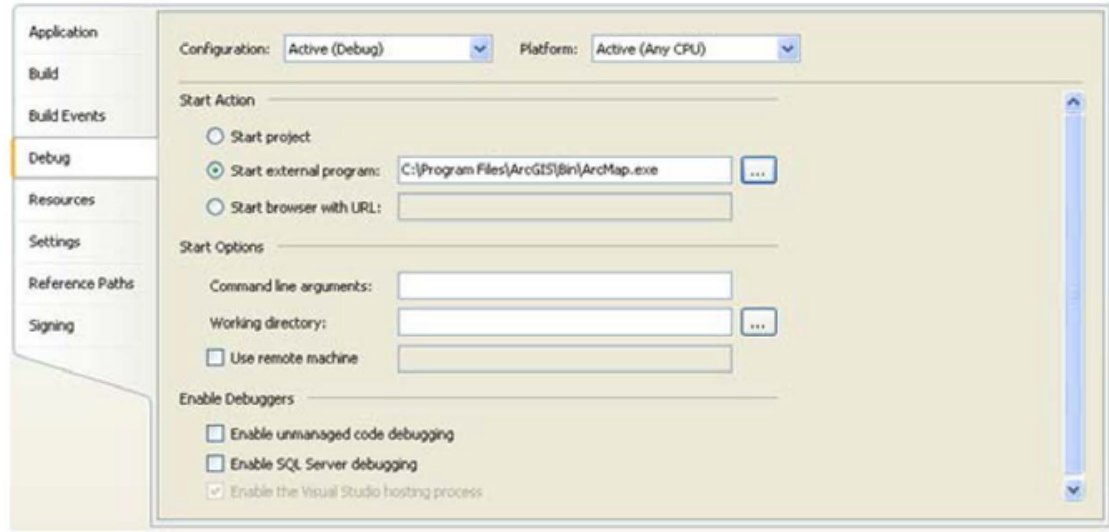

The debugging preferences are not set in the associate sample files since that information is stored in the project user file (*.csproj.user or *.vbproj.user) and those files are not included with the samples. If you are trying to debug using the provided sample, set ArcMap.exe as the debug application.

If the project user file has been removed since you created the project, Start Action will be set to Start project. To debug, set it to Start external application and browse to ArcMap.exe.

With the default installation settings, ArcMap.exe is installed in \Program Files\ArcGIS\Bin.

3. Open the code window for the ZoomToLayer file. Find the class constructor (ZoomToLayer() in C\#, New in VB NET) and set a breakpoint. See the following screen shot: 
Create a command by inheriting from BaseCommand

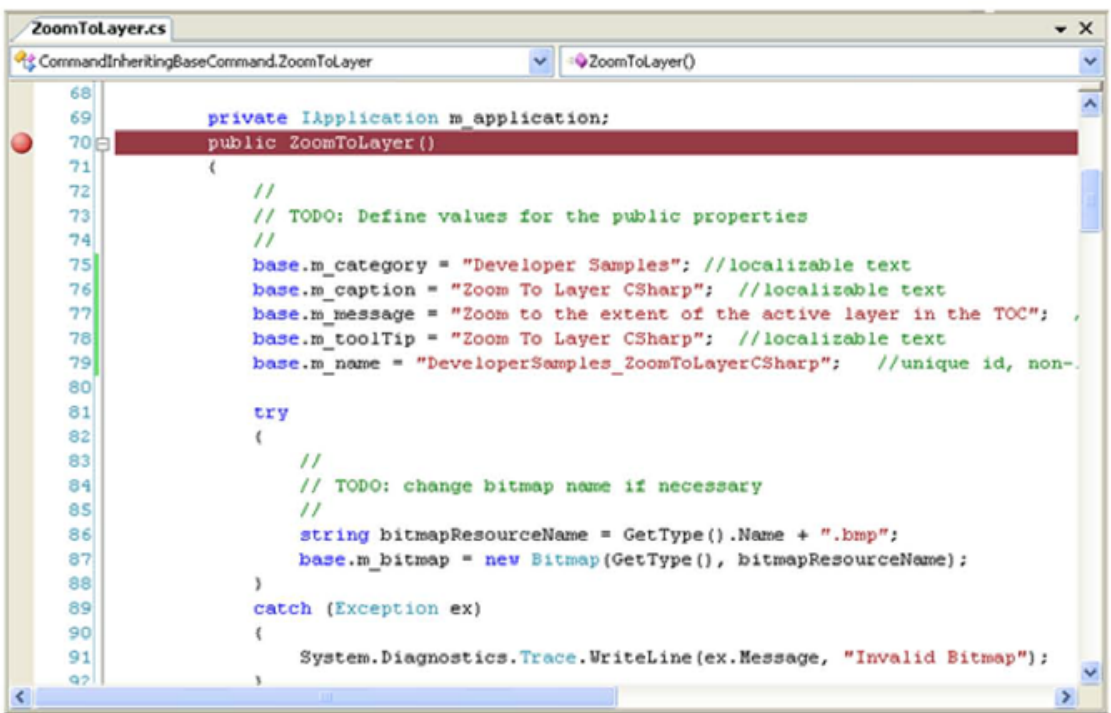

To set a breakpoint in the Visual Studio 2005 IDE, click the Margin Indicator bar (gray area on the left side of the code editor) beside the code where you want the breakpoint set.

4. Click the Debug menu and click Start Debugging (or press F5); Visual Studio runs ArcMap. Follow the same steps in Use the command in ArcMap section to go over the code. When the ZoomToLayer class is instantiated, the debugger switches to Visual Studio 2005 and allows you to step through the code one line at a time with the buttons on the Debugging toolbar.

\section{Deploying the command}

Once you have created your command, run it on another user's ArcGIS Desktop installation. To do this, deploy your command. For more information, see Deploving in-process components (DLLs).

\section{See Also:}

How to implement custom commands and tools

Sample: Create a command by inheriting from BaseCommand

Deploving in-process components (DLLs) 



\title{
Appendix D. "How to create a custom install program" Documentation from the ESRI Resource Center
}

How to create a custom install program

\author{
How to create a custom install program
}

This document was published with ArcGIS 9.3.

A 2.2 version also exists.

Summary

To deploy stand-alone applications (.exe) or in-process components (.dll), you can create a custom installation program using Visual Studio or third-party installation software such as Wise or InstallShield. By using Visual Studio, it is possible to create a custom install program that deploys and registers your components-the key being the System.Runtime.InteropServices. RegistrationServices class. This class has the RegisterAssembly and

UnregisterAssembly members, which register and unregister managed classes with component object models (COM). These are the same functions the RegAsm utility uses. Using these functions inside a custom installer class along with a setup program is the complete solution.

\begin{tabular}{|l|l|}
\hline Development licensing & Deployment licensing \\
\hline Engine Developer Kit & Engine Runtime \\
\hline ArcView & ArcView \\
\hline ArcEditor & ArcEditor \\
\hline ArcInfo & ArcInfo \\
\hline
\end{tabular}

\section{Deploying a .NET component customization}

The steps in this article show how to deploy a .NET component customization; for example, a class library developed in .NET that provides custom commands and tools.

The technique described in this article assumes that ArcGIS is installed on the target machine.

1. In Visual Studio, open a .NET solution that already contains a class library project with at least one class exposed to COM; for example, a custom command class. You may want to create your command using Visual Studio IDE Integration features of the .NET SDK.

For more information on using the Visual Studio IDE integration to create you own custom ArcGIS based COM components see About the ArcGIS item templates.

2. In the Solution Explorer, right click on the project name and choose 'Add' > 'New Item' and then choose 'Installer Class' from the New Item dialog. See the following screen shot:

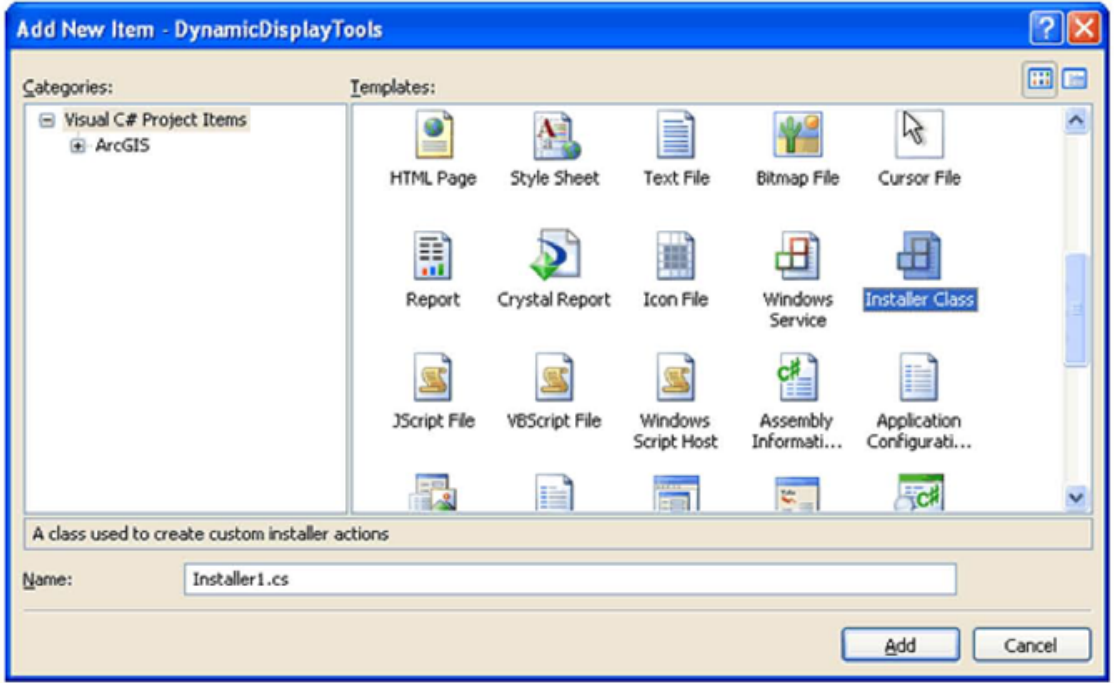


How to create a custom install program

You will need to have a name for your class other than "Installer" as that is the name of the class in

System.Configuration. Install that your install class will inherit from.

3. The next step is to override the install and uninstall functions implemented in the installer base class. You use the RegistrationServices class RegisterAssembly and UnregisterAssembly methods to register the components. Make sure you use the SetCodeBase flag to indicate that the code base key for the assembly is set in the registry. The RegistrationServices class is found in the System.Runtime.InteropServices namespace. To accomplish this, first add a new imports or using statement at the top of the class. See the following:

[C\#]

using System.Runtime.Interopservices;

[VB.NET]

Imports system.Runtime.Interopservices

Then, add the following code inside of the installer class: [C\#]

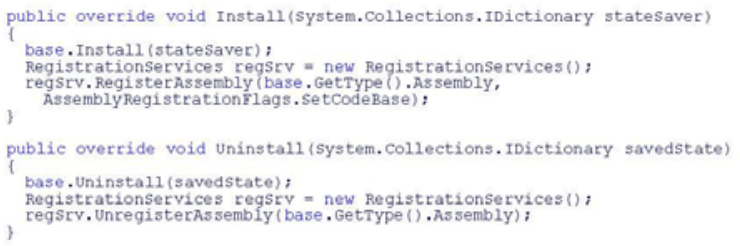

[VB.NET]

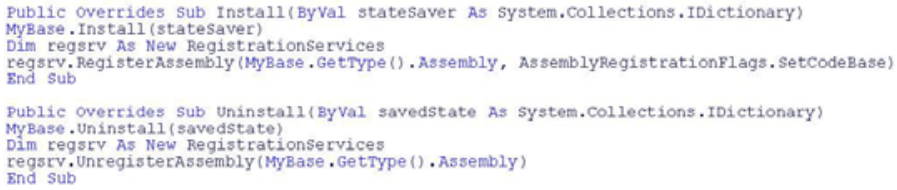

4. At this point you must re-build your project to ensure that the latest version of all the project files get included in the setup program. Choose Build > Rebuild Solution from the Visual Studio menus.

If you want your application to be able to work across different versions of assemblies, you may need to specify that in the properties of any referenced assemblies. You can do this by clicking on a specific assembly in the References section of the Solution Explorer and setting the 'Specific Version' to False. Do this before you rebuild your project.

5. Add a setup program to your solution by choosing 'File' > 'New Project' from the menus. On the Add New Project dialog box, select Setup and Deployment in the Project types area, then click Setup Project in the Templates area. Name the new project and click OK to add the new setup project to your solution. See the following screen shot:

http://resources.esri.com/help/9.3/ArcGISDesktop/dotnet/Odf20605-b457-42d6-b63c-341a3824474a.htm[9/2/2009 5:27:10 PM] 
How to create a oustom install prog am

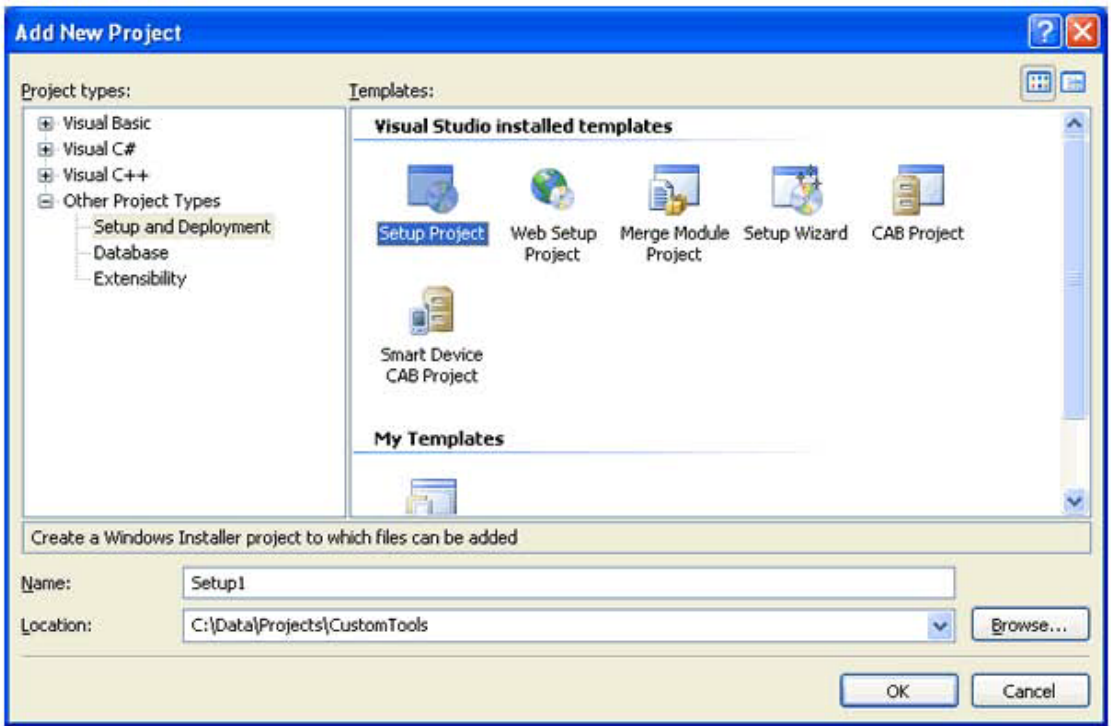

6. In the Solution Explorer, right-click the new Setup project, click Add, then select Project Output. Choose the project you want to deploy. Select Primary output and click OK. See the following screen shot:

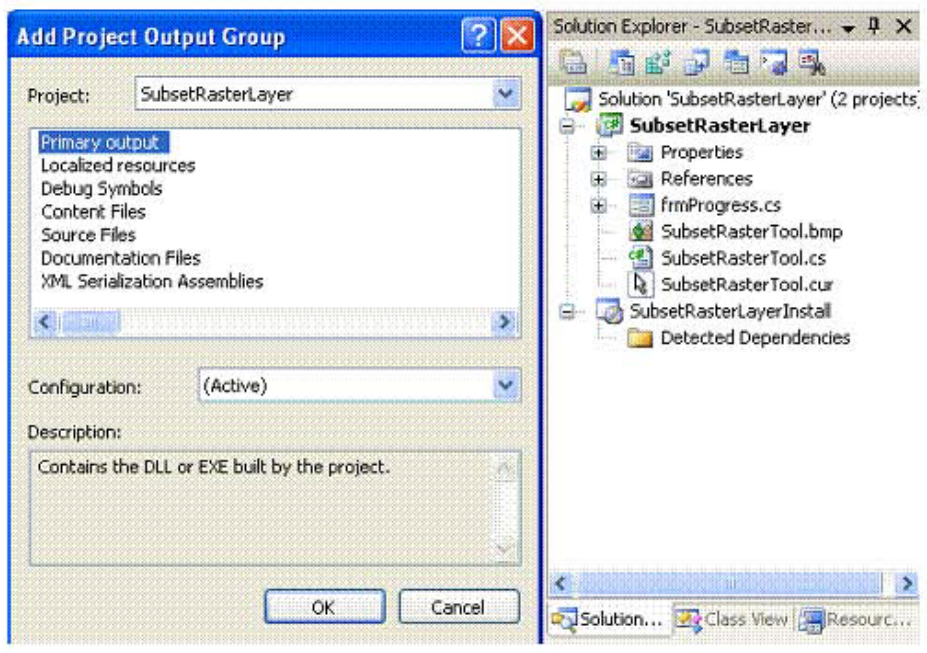

7. From the list of detected dependencies that is regenerated, exclude all references to ESRI primary interop assemblies; for example, ESRI .ArcG IS.System. Also exclude the Stdole.dll reference. The only items typically left in the list are the $\forall b$ file, Microsoft. NET Framework file, and the Primary output from the $<$ AssemblyName><Version> file, which represents the dill or .exe you are compiling. See the following screen shot:

http://resources.esri.com/help/9.3/ArcGISDesktop/dotnet/odf20605-b457-42d6-b63c-341a3824474a.htm[9/2/2009 5:27:10 PM] 
How to create a custom install program

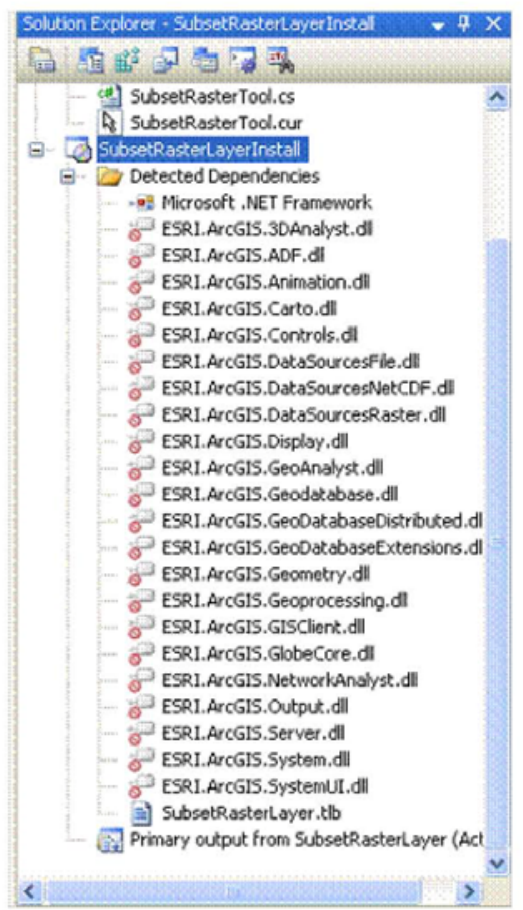

You should never include ArcGIS assemblies in your deployment package as these are installed by the ArcGIS installation program.

8. Additionally, you need to change the value of the Register property for the .tlb file. By default, the .tlb file is set to be registered with COM at install time. However, this causes an error during installation. To accomplish this, right-click the .tlb file in the project's detected dependencies tree node. In the Properties pane, set the value of the Register property to vsdrfDoNotRegister before compiling the installer. See the following screen shot: 
How to create a oustom irstall prog am

\begin{tabular}{|c|c|c|}
\hline \multicolumn{2}{|l|}{ Properties } & X \\
\hline \multicolumn{2}{|c|}{ SubsetRasterLayer.tlb File Installation Properties } & - \\
\hline \multicolumn{3}{|l|}{ 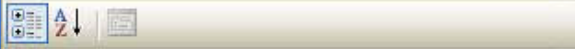 } \\
\hline Condition & & $\wedge$ \\
\hline Exclude & False & \\
\hline Folder & Application Folder & \\
\hline Hidden & False & \\
\hline PackageAs & vsdpaDefault & \\
\hline Permanent & False & \\
\hline Readonly & False & \\
\hline Register & vsdrfDoNotRegister & \\
\hline SharedLegacyFile & False & \\
\hline SourcePath & C:\{arcgis\}'Samples Net'Subse & \\
\hline System & False & \\
\hline TargetName & SubsetRasterLayer.tlb & \\
\hline Traneitive & Faleo & 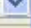 \\
\hline \multicolumn{3}{|c|}{$\begin{array}{l}\text { Register } \\
\text { Specifies whether a file should be registered during installation }\end{array}$} \\
\hline PProperties 0 & & \\
\hline
\end{tabular}

9. The final steps involve associating the custom installation steps configured in the new installer class with the sebup project. To do this, right-click the setup project in the Solution Explorer, choose view, then choose Custom Actions. See the following screen shot:

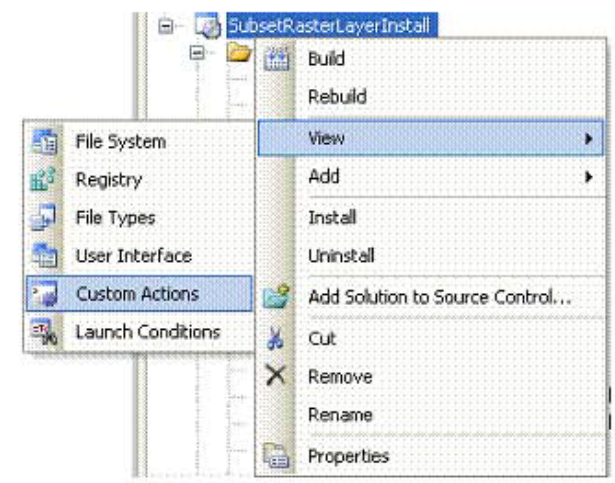

10. In the resulting custom actions for the installation project, right-click the Install folder and choose Add Custom Action. See the following screen shot:

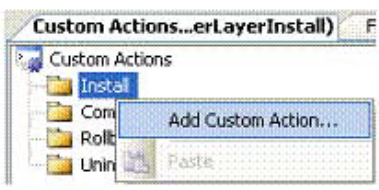

11. On the Select Item in Project dialog box, double-click Application Folder. See the following screen shot:

http://r esources.esri.com/help/9.3/ArcGISDesktop/dotnet/odf20605-b457-42d6-b63c-341a3824474a.htm[9/2/2009 5:27:10 PM] 
How to create a oustom install prog am

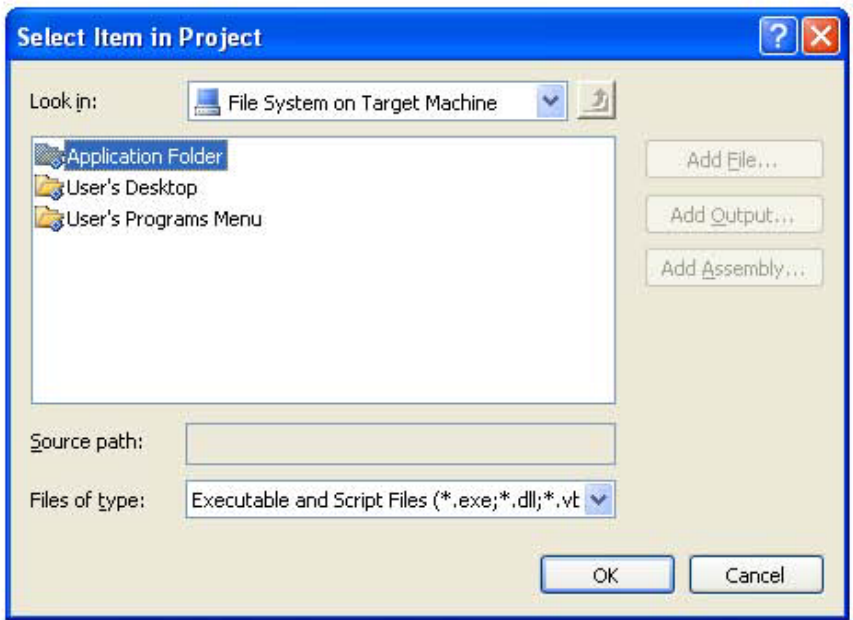

12. From the Application Folder, double-click the Primary output from the <Assembly Name $><$ Version $>$ file to associate the custom install function created earlier with the setup's custom install action. See the following screen shot:

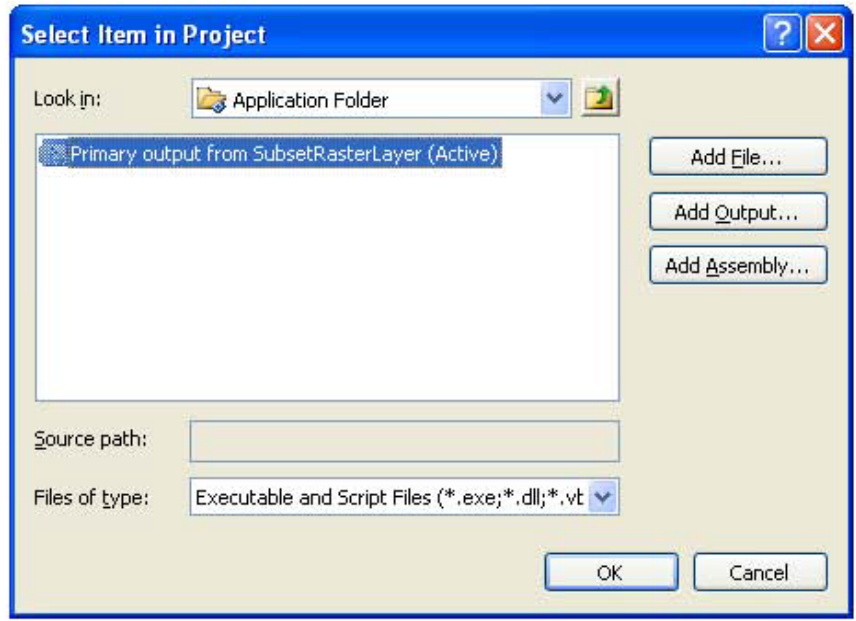

13. Repeat this process for the setup's uninstall. See the following screen shot: 


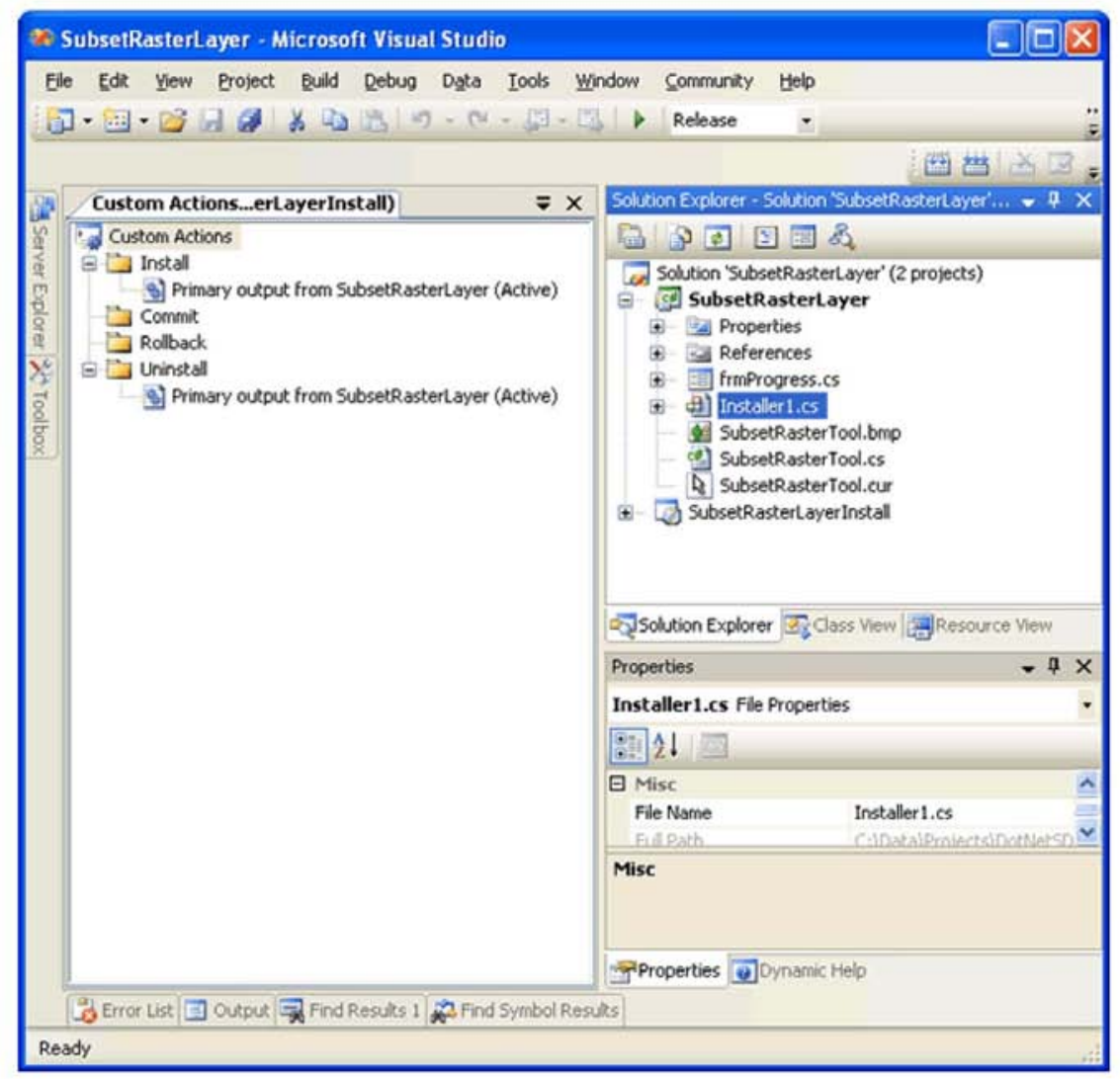

14. Rebuild the entire solution to generate the setup's executable.

A setup project is not built by default. In the Solution Explorer, you can right-click the setup project and click Build to build the setup project. You can also right-click the solution and choose Configuration Manager, then on the Configuration Manager dialog box, select the Build option for the setup project before rebuilding the solution.

Running the setup executable on a target machine installs the components and registers them with COM. The COMRegisterFunction routine then registers the components in the appropriate component categories.

Please note that you must uninstall the custom component before you attempt to uninstall ArcGIS. The reason for that is that custom components need to loaded at install/uninstall time. When loading the .NET Framework loads the custom component it must also load all referenced assemblies (eg "ESRI.ArcGIS.ArcMap.dII" and

"ESRI.ArcGIS.System.dII"). If these referenced assemblies are missing at install/uninstall time then the install/uninstall will fail.

\section{See Also:}

How to register. NET components with COM How to register classes in COM component categories About the ArcGIS item templates 